Distribution Category:

Mathematics and

Computer Science (UC-405)

ANL-95/18

\title{
Users Guide to the PGAPack Parallel Genetic Algorithm Library
}

by

David Levine

Mathematics and Computer Science Division

This work was supported in part by the Mathematical, Information, and Computational Sciences Division subprogram of the Office of Computational and Technology Research, U.S. Department of Energy, under Contract W-31-109-Eng-38. 


\section{DISCLAIMER}

Portions of this document may be illegible in electronic image products. Images are produced from the best available original document. 


\section{Contents}

0 Quick Start $\quad 1$

I Getting Started $\quad 2$

1 Introduction $\quad 3$

2 Installation

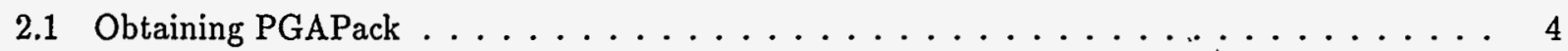

2.2 Requirements . . . . . . . . . . . . . . . . . . 4

2.3 Structure of the Distribution Directory . . . . . . . . . . . . . . . 4

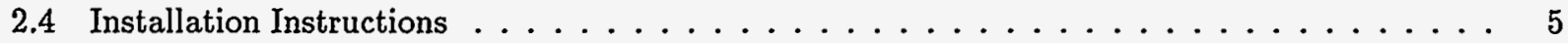

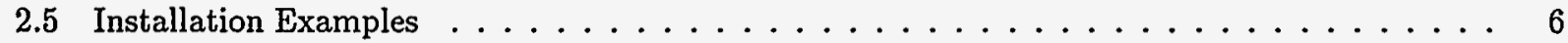

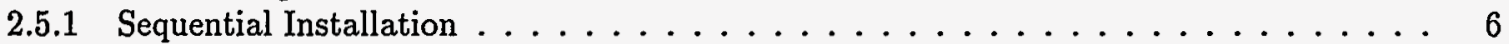

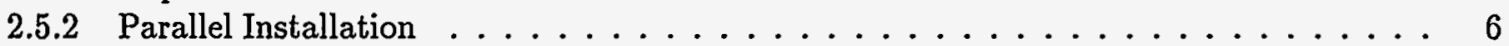

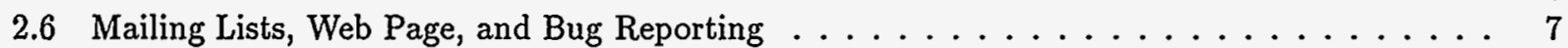

3 Examples $\quad-\quad 8$

3.1 Maxbit Problem in C . . . . . . . . . . . . . . . . . . 8

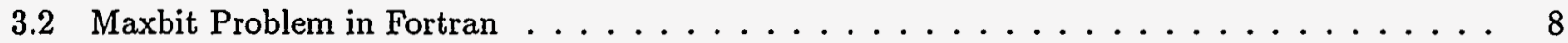

3.3 Specifying Nondefault Values $\ldots \ldots \ldots \ldots \ldots \ldots \ldots \ldots \ldots$

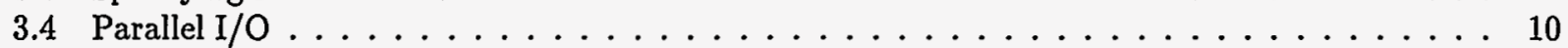

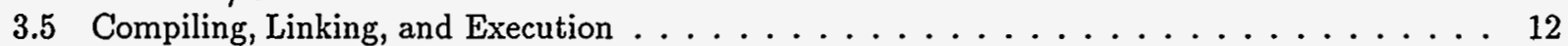

II Users Guide $\quad 14$

4 The Structure of PGAPack 15

4.1 Native Data Types . . . . . . . . . . . . . . . . . . . . . 15

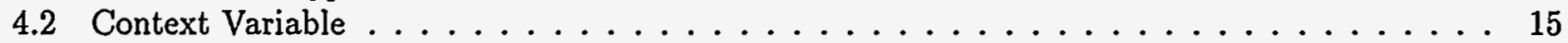

4.3 Levels of Usage Available . . . . . . . . . . . . . . . . . . . . 15

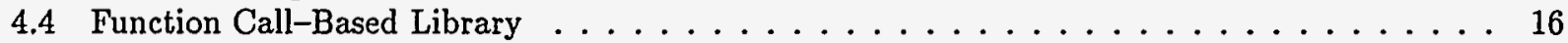

4.5 Header File and Symbolic Constants . . . . . . . . . . . . . . . . 16

4.6 Evaluation Function $\ldots \ldots \ldots \ldots \ldots \ldots \ldots \ldots \ldots \ldots \ldots$

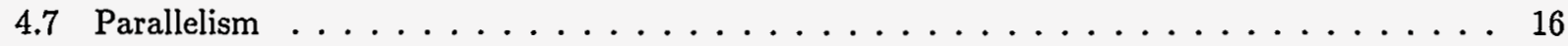

4.8 Implementation . . . . . . . . . . . . . . . . . 17

5 Basic Usage 18

5.1 Required Functions . . . . . . . . . . . . . . . . . . 18

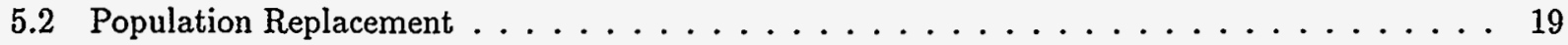

5.3 Stopping Criteria . . . . . . . . . . . . . . . . . . . 21

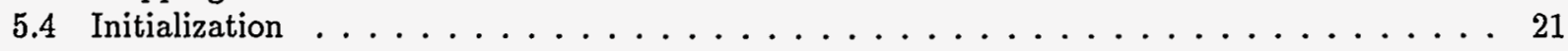

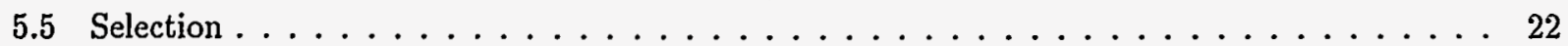




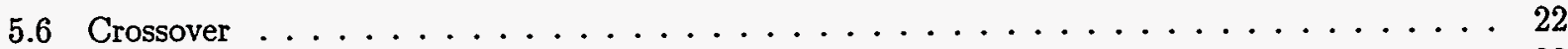

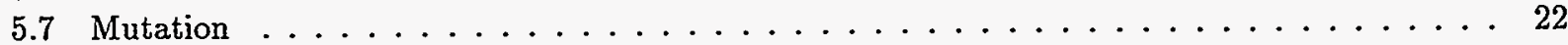

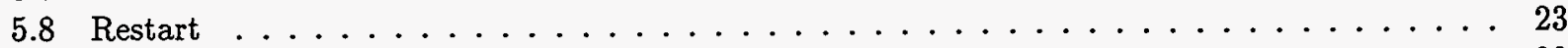

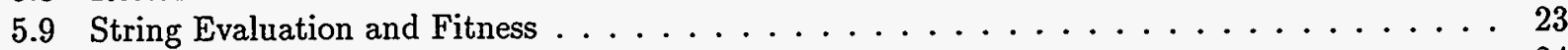

5.10 Accessing Allele Values . . . . . . . . . . . . . . . . . . . . . 24

5.10 .1 Representing an Integer with a Binary String . . . . . . . . . . . . . . 25

5.10 .2 Representing a Real Value with a Binary String . . . . . . . . . . 25

5.10 .3 Example. . . . . . . . . . . . . . . . . . . . . . 26

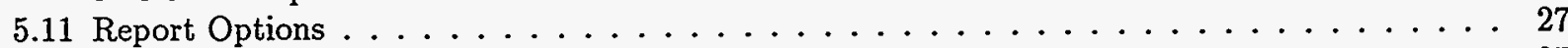

5.12 Utility Functions . . . . . . . . . . . . . . . . . . . 27

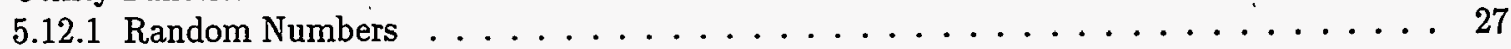

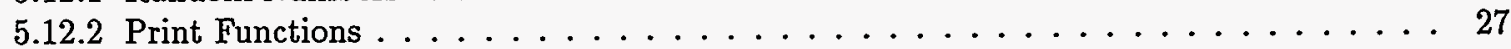

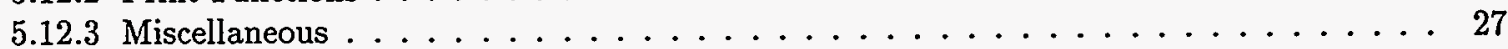

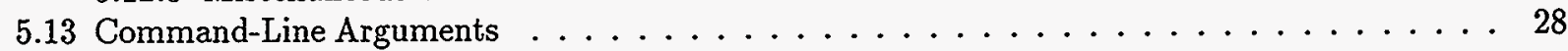

6 Explicit Usage $\quad 29$

6.1 Notation . . . . . . . . . . . . . . . . . . . . . . . 29

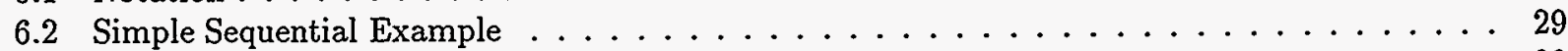

6.3 Complex Example . . . . . . . . . . . . . . . . . . . . 30

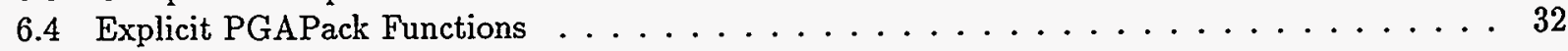

7 Custom Usage: Native Data Types 33

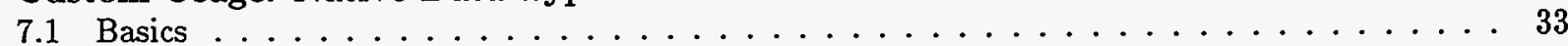

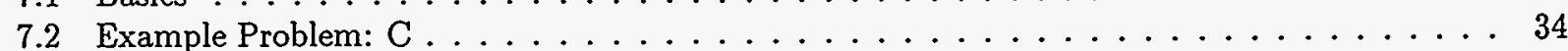

7.3 Example Problem: Fortran $\ldots \ldots \ldots \ldots \ldots \ldots \ldots \ldots \ldots \ldots$

8 Custom Usage: New Data Types 37

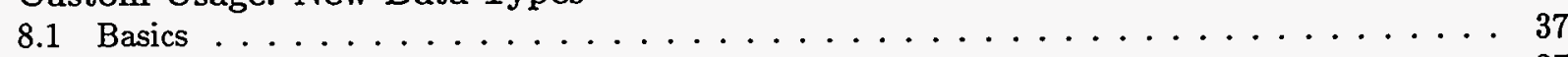

8.2 Example Problem . . . . . . . . . . . . . . . . . 37

9 Hill-Climbing and Hybridization $\quad 44$

10 Parallel Aspects $\quad 46$

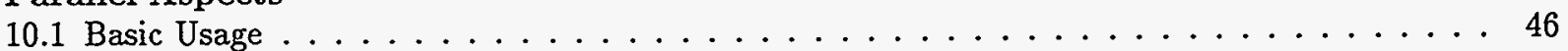

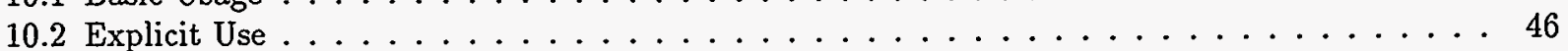

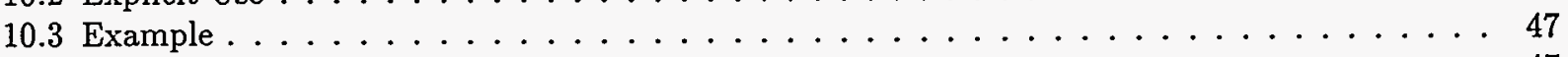

10.4 Performance . . . . . . . . . . . . . . . . . . . . 47

11 Fortran Interface $\quad 49$

12 Debugging Tools $\quad 51$

III Appendixes $\quad 54$

A Default Values $\quad \mathbf{5 5}$

B Function Bindings $\quad 57$

C Parallelism Background $\quad 64$

$\begin{array}{ll}\text { D Machine Idiosyncrasies } & 68\end{array}$

$\begin{array}{ll}\text { E Common Problems } & 71\end{array}$

$\begin{array}{ll}\text { Acknowledgments } & 73\end{array}$

$\begin{array}{lc}\text { Bibliography } & \mathbf{7 4}\end{array}$ 


\section{Chapter 0}

\section{Quick Start}

If you wish to get started by just typing a few lines and running an example, this section is for you. We assume you have ftped the compressed tar file pgapack.tar. $Z$ containing the distribution into /home/username. To build a sequential version of PGAPack for a Sun SparcStation in /usr/local/pga and run a test example, type

1. uncompress /home/username/pgapack.tar.Z

2. mkdir/usr/local/pga

3. cd /usr/local/pga

4. $\operatorname{tar}$ xvf /home/username/pgapack.tar

5. configure -arch sun4

6. make install

7. /usr/local/pga/examples/c/maxbit

To build an optimized (no built-in debugging capabilities), parallel version of PGAPack for an IBM SP parallel computer, using an MPI implementation with include files in /uss/local/mpi/include and library in /usr/local/mpi/lib, and run a test example using four processes, type

1. uncompress/home/username/pgapack.tar.Z

2. mkdir/usr/local/pga

3. cd /usr/local/pga

4. tar xvf /home/username/pgapack.tar

5. configure -arch rs6000 \

-mpiinc/usr/local/mpi/include -mpilib /usr/local/mpi/lib/libmpi.a

6. make install

7. mpirun -np 4 /uss/local/pga/examples/c/maxbit

Step 7, the execution step, is completely dependent on the MPI implementation. This example uses the mpirun script that is distributed with the MPICH implementation [1]. Other MPI implementations may have other ways to specify the number of processes to use.

More details on the installation process and various options are given in Chapter 2. Chapter 3 (example problems) and Sections 5.1 (required functions) and 5.9 (string evaluation and fitness) should be read next. 


\section{Part I}

\section{Getting Started}




\section{Chapter 1}

\section{Introduction}

PGAPack is a parallel genetic algorithm library that is intended to provide most capabilities desired in a genetic algorithm package, in an integrated, seamless, and portable manner. Key features of PGAPack are as follows:

- Ability to be called from Fortran or C.

- Executable on uniprocessors, multiprocessors, multicomputers, and workstation networks.

- Binary-, integer-, real-, and character-valued native data types.

- Object-oriented data structure neutral design.

- Parameterized population replacement.

- Multiple choices for selection, crossover, and mutation operators.

- Easy integration of hill-climbing heuristics.

- Easy-to-use interface for novice and application users.

- Multiple levels of access for expert users.

- Full extensibility to support custom operators and new data types.

- Extensive debugging facilities.

- Large set of example problems. 


\section{Chapter 2}

\section{Installation}

\subsection{Obtaining PGAPack}

The complete distribution of PGAPack is available by anonymous ftp from ftp.mcs.anl.gov in the file pub/pgapack/pgapack.tar.Z. The distribution contains all source code, installation instructions, this users guide, and a collection of examples in $\mathrm{C}$ and Fortran. The current release of PGAPack is 1.0. You can check which version of PGAPack you have by running any $C$ language PGAPack program with the command-line option -pgaversion.

\subsection{Requirements}

To compile PGAPack, you must have an ANSI $\mathrm{C}$ compiler that includes a full implementation of the Standard $\mathrm{C}$ library and related header files. If you wish only to build a sequential version of PGAPack this is all that is required.

To build a parallel version, you must have an implementation of the Message Passing Interface (MPI) $[5,6]$ for the parallel computer or workstation network you are running on. If you do not have a native version of MPI for your computer, several machine-independent implementations are available. Most of the testing and development of PGAPack was done by using the MPICH implementation of MPI which is freely available [1].

\subsection{Structure of the Distribution Directory}

The PGAPack distribution contains the following files and subdirectories:

- CHANGES: Changes new to this release of PGAPack.

- COPYRIGHT: The usage terms.

- README: General instructions, including how to build and install PGAPack.

- configure.in: The "source code" for the configure script.

- configure: A Unix shell script that configures Makefile.in for a specific architecture.

- Makefile.in: Prototype makefile that is configured into the file Makefile for a specific architecture by configure.

- docs: The users guide and any other supporting files.

- examples: A directory containing $\mathrm{C}$ and Fortran examples.

- include: The PGAPack include directory. 
- lib: The top-level directory where PGAPack will be installed.

- man: The directory containing the PGAPack man pages.

- source: The source code for PGAPack.

In the rest of this guide we use ":" as the top-level directory, e.g., ./source, ./examples/c/maxbit.c.

\subsection{Installation Instructions}

When installing PGAPack you make two choices: whether to build a sequential (the default) or parallel version (see the flags -mpiinc and -mpilib below) and whether to build an optimized (the default) or debug version (the - debug flag). In broad outline, the installation steps are as follows.

1. Make a directory to install PGAPack in (mkdir/usr/local/pga).

2. Change directories to the directory created in the last step (cd /usr/local/pga).

3. Obtain the compressed tar file pgapack.tar. $Z$ by anonymous ftp from ftp.mcs.anl.gov in the directory pub/pgapack.

4. Uncompress the tar file (uncompress pgapack.tar.Z).

5. Untar the uncompressed PGAPack tar file (tar xvf pgapack.tar).

6. Use configure to configure the makefiles (configure -arch ARCH_TYPE)

where ARCH_TYPE is one of sun4 for Sun SparcStations workstations, next for NeXT workstations, rs6000 for IBM RS/6000 workstations, irix for Silicon Graphics workstations, hpux for Hewlett Packard workstations, alpha for DEC Alpha workstations, linux for machines running Linux, freebsd for machines running FreeBSD, generic for generic 32-bit machines, powerchallenge for the Silicon Graphics Power Challenge Array, challenge for the Silicon Graphics Challenge, $t 3 d$ for the Cray T3D, sp2 for the IBM SP2, paragon for the Intel Paragon, or exemplar for the Convex Exemplar.

The full configure options are configure -arch ARCH_TYPE [-cc cC] [-cflags CFLAGS] [-f77 FC] [-fflags FFLAGS] [-debug] [-mpiinc MPI_INCLUDE_DIRECTORY] [-mpi]ib MPI_LIBRARY] [-help] where all parameters except -arch are optional and do the following:

- -cc; The name of the ANSI $\mathrm{C}$ compiler, cc by default.

- -cflags: Options passed to the $\mathrm{C}$ compiler.

- -f77: The name of the Fortran 77 compiler, $₫ 77$ by default. (The Fortran compiler is used only to compile the Fortran examples in the ./examples/fortran directory.)

- -fflags: Options passed to the Fortran compiler.

- -debug: If specified, enables the debugging features (see Chapter 12) and compiles the source code with the $-\mathrm{g}$ flag. If this flag is not specified the debugging features are disabled, and the library is compiled with the -0 flag

- -mpiinc: The directory where MPI include files are located.

- -mpilib: The full path to the MPI library.

If -mpiinc and -mpilib are specified, a parallel version of PGAPack will be built. If these flags are not specified, a sequential version of PGAPack will be built.

7. Execute the makefile (make install).

8. Add PGAPack's man pages to your man page path. (setenv MANPATH "\$MANPATH"":/home/pgapack/ man")

9. Execute a test problem 
- /usr/local/pga/examples/c/maxbit in C

- /usr/local/pga/examples/fortran/maxbit in Fortran.

If a parallel version of PGAPack was used, the actual commands to execute a parallel program in Step 9 will depend on the particular MPI implementation and parallel computer used. See Appendix D for some examples.

\subsection{Installation Examples}

These installation examples assume you have ftped the compressed tar file pgapack.tar. $Z$ containing the distribution into /home/username.

\subsubsection{Sequential Installation}

To build a sequential version of PGAPack for a Sun SparcStation in /usr/local/pga and run a test example, type:

1. uncompress /home/username/pgapack.tar.Z

2. mkdir/usr/local/pga

3. cd /usr/local/pga

4. tar xvf /home/username/pgapack.tar

5. configure -arch sun4

6. make install

7. /usx/local/pga/examples/c/maxbit

\subsubsection{Parallel Installation}

To build an optimized (no built-in debugging capabilities), parallel version of PGAPack for an IBM SP parallel computer using an MPI implementation with include files in /usr/local/mpi/include and library in /usr/local/mpi/lib, and run a test example using four processes, type:

1. uncompress/home/username/pgapack.tar.z

2. mkdir /usr/local/pga

3. cd /usr/local/pga

4. tar xvf /home/username/pgapack.tar

5. configure -arch $\operatorname{rs} 6000 \backslash$ -mpiinc/usr/local/mpi/include -mpilib/usr/local/mpi/lib/libmpi.a

6. make install

7. mpirun -np $4 /$ usr/local/pga/examples/c/maxbit

Step 7, the execution step, is completely dependent on the MPI implementation. This example uses the mpirun script that is distributed with the MPICH implementation [1]. Other MPI implementations may have other ways to specify the number of processes to use. 


\subsection{Mailing Lists, Web Page, and Bug Reporting}

To join the PGAPack mailing list to receive announcements of new versions, enhancements, and bug fixes, send electronic mail to pgapack@mcs . anl.gov. Bug reports should be sent to pgapack-bugs@mcs.anl.gov. The World Wide Web page for PGAPack is http://www.mcs.anl.gov/pgapack.html and contains up-todate news and a list of bug reports.

When reporting a bug, please include as much information and documentation as possible. Helpful information would include PGAPack version number (-pgaversion), MPI implementation and version used, configuration options, type of computer system, problem description, and error message output. It is helpful if you put a PGAPrintContextVariable call before and after the PGASetUp call. Additionally, if possible, build a debug version of PGAPack and send "high-level" output from running your program with the trace facility enabled (Chapter 12). 


\section{Chapter 3}

\section{Examples}

This chapter presents some simple PGAPack programs. The problem chosen is the Maxbit problem. The objective is to maximize the number of 1-bits in a string.

Section 3.1 presents a simple PGAPack program in $C$ whose structure is sufficient to solve many problems. Section 3.2 presents this same program in Fortran. Section 3.3 shows how to change default values in PGAPack. Section 3.4 contains an example that shows how keyboard input may be read in an MPI environment. Finally, Section 3.5 shows how to compile, link, and execute a PGAPack program. These and other examples may be found in the ./examples/c and ./examples/fortran directories.

\subsection{Maxbit Problem in $\mathbf{C}$}

Figure 3.1 shows a minimal program and evaluation function in $\mathrm{C}$ for the Maxbit problem. All PGAPack $\mathrm{C}$ programs must include the header file pgapack.h. The PGACreate call is always the first function called in a PGAPack program. It initializes the context variable, ctx. The parameters to PGACreate are the arguments to the program (given by argc and argv), the data type selected (PGADATATYPE BINARY), the string length (100), and the direction of optimization (PGA MAXIMIZE). The PGASetUp call initializes all parameters and function pointers not explicitly set by the user to default values.

PGARun executes the genetic algorithm. Its second argument is the name of a user-defined function (evaluate) that will be called to evaluate the strings. PGADestroy releases all memory allocated by PGAPack. Note that all PGAPack functions take the context variable as an argument (except PGACreate, which creates the context variable).

The evaluate function must be written by the user, must return a double, and must follow the exact calling sequence shown. PGAGetStringLength returns the string length. PGAGetBinaryAllele returns the value of the ith bit of string $p$ in population pop.

\subsection{Maxbit Problem in Fortran}

The Fortran Maxbit problem in Figure 3.2 is similar to the $\mathrm{C}$ version in Figure 3.1. The Fortran include file is pgapackf.h and should be included in every Fortran function or subroutine that makes PGAPack calls $^{1}$. Since Fortran provides no standard mechanism for specifying command line arguments, these are omitted from the PGACreate function call. The context variable, ctx, is declared integer in Fortran.

The evaluation function evaluate must contain exactly the calling sequence shown and must return a double precision value. Note that evaluate is declared in an external statement in the program unit in which it is used as an actual argument. This is a requirement of the Fortran language. In Fortran, the range of allele values is 1 :stringlen, rather than 0 :stringlen-1 as in C.

\footnotetext{
${ }^{1}$ Since not all Fortran compilers support the -I mechanism for specifying the include file search path, you will need to copy or set up a symbolic link to pgapackf .h from the directory you are compiling a Fortran program in.
} 
\#include "pgapack.h"

double evaluate (PGAContext *ctx, int $\mathrm{p}$, int pop);

int main(int argc, char **argv)

\{

PGAContext *ctx;

ctx = PGACreate (\&argc, argv, PGA_DATATYPE_BINARY, 100, PGA_MAXIMIZE);

PGASETUP

(ctx

PGARun (ctx, evaluate

PGADestroy (ctx

return;

\}

double evaluate (PGAContext *ctx, int $\mathrm{p}$, int pop)

\{

int $i$, nbits, stringlen;

stringlen = PGAGetStringLength $(\operatorname{ctx})$;

nbits $=0$;

for ( $i=0$; i<stringlen; $i++$ )

if (PGAGetBinaryAllele(ctx, p, pop, i)) noitst+;

return((double) nbits);

\}

Figure 3.1: PGAPack C Program for the Maxbit Example

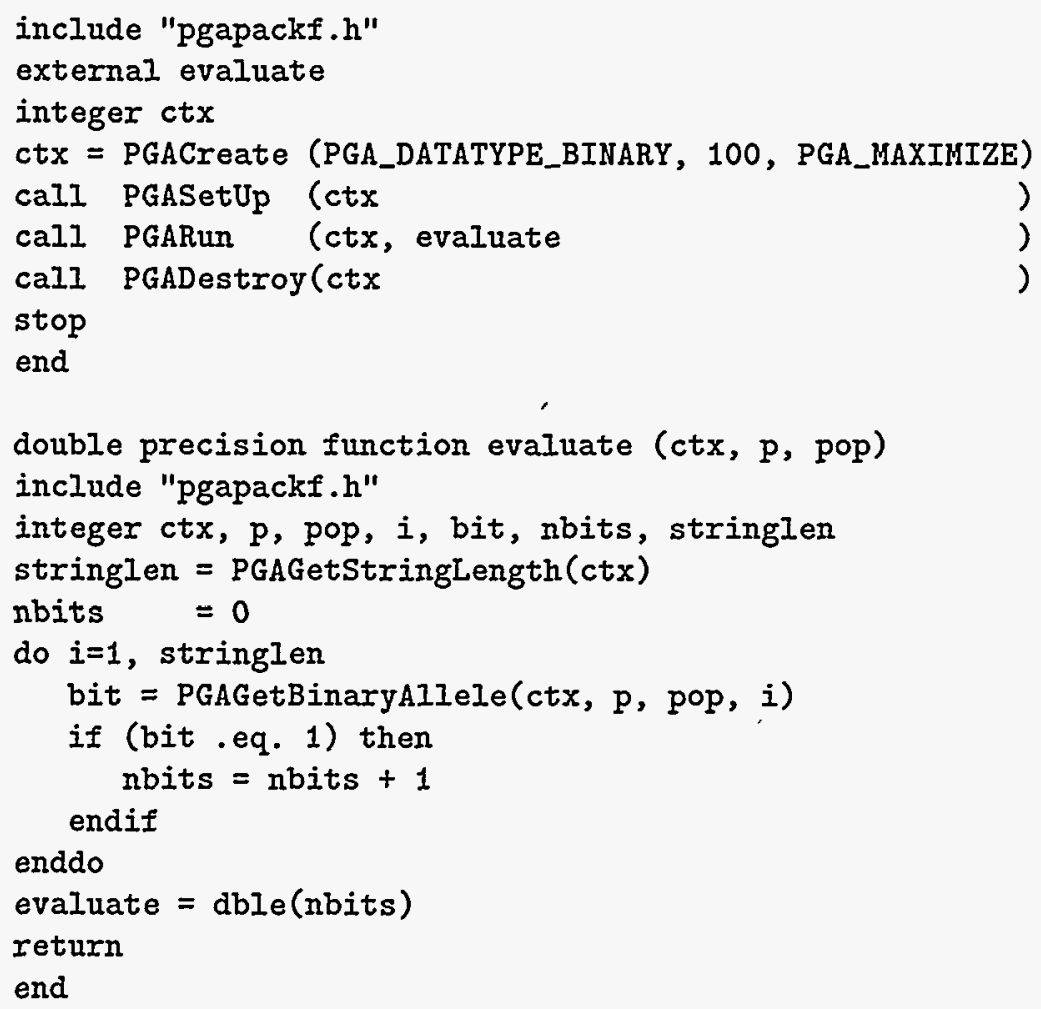

Figure 3.2: PGAPack Fortran Program for the Maxbit Example 


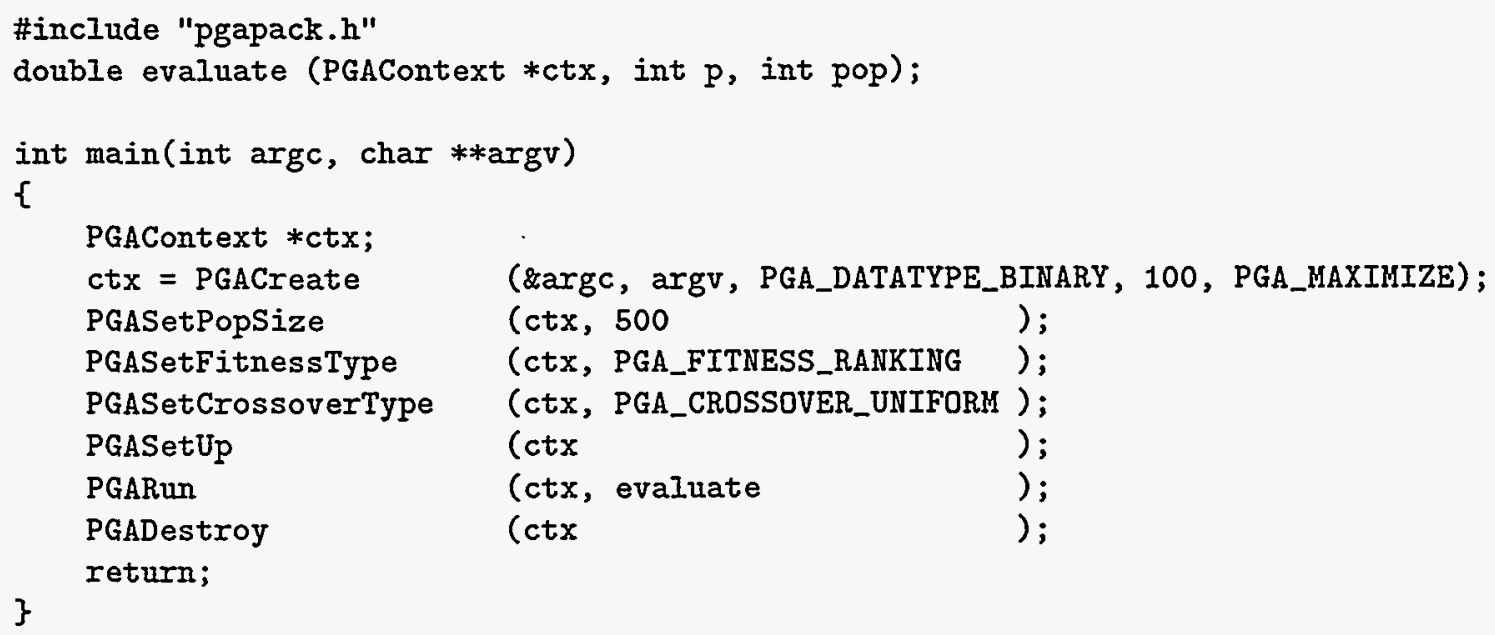

Figure 3.3: Specifying Nondefault Values

\subsection{Specifying Nondefault Values}

PGAPack offers a wide range of choices for parameter values, operators, and algorithmic choices. These will be set to default values in PGASetUp if the user does not explicitly set a value for them. A nondefault value may be set by using the PGASet family of calls after PGACreate has been called, but before PGASetUp has been called.

In Figure 3.3 the PGASet calls change the default values for population size, fitness calculation, and crossover type. PGASetPopSize changes the population size to 500. PGASetFitnessType specifies that the fitness values be determined by using a ranking procedure rather than by direct use of the evaluation function values. PGASetCrossoverType specifies that uniform crossover, rather than the default of two-point crossover is to be used. Most PGASet calls are discussed in Chapter 5.

\subsection{Parallel I/O}

The examples in Figures 3.4 (C) and 3.5 (Fortran) read values for the two parameters len (string length) and maxiter (maximum number of GA iterations) from standard input. These examples will work correctly with either a sequential or parallel version of PGAPack. However, the explicit use of MPI calls for I/O is necessary only if a parallel version of PGAPack is used, and parameter values are read from standard input. The purpose is to be sure that each process receives a copy of the input values. See Appendix $C$ for further details.

MPI Init (\&argc, \&argv) is always the first function called in any MPI program. Each process executes MPI_Comm_rank (MPI_COMM_WORLD, \&my id) to determine its unique rank in the communicator ${ }^{2}$ MPI_COMM_WORLD. The logic used in this program is to have process 0 read and write from/to standard input/output and broadcast (using MPI Bcast) the parameters to the other processes. The PGAPack function calls are similar to those in the previous examples. If the user called MPI Init, the user must also call MPIFinalize before exiting.

We elaborate here on the MPI_Bcast function because of its practical value in the model of parallel I/O shown. For more detailed discussion of MPI concepts and functions, the user should consult $[5,6]$.

The $\mathrm{C}$ binding for MPI_Bcast is int MPI_Bcast (void *buf, int count, MPI_Datatype datatype, int root, MPI_Comm comm) and the Fortran binding

\footnotetext{
${ }^{2}$ See Appendix C
} 


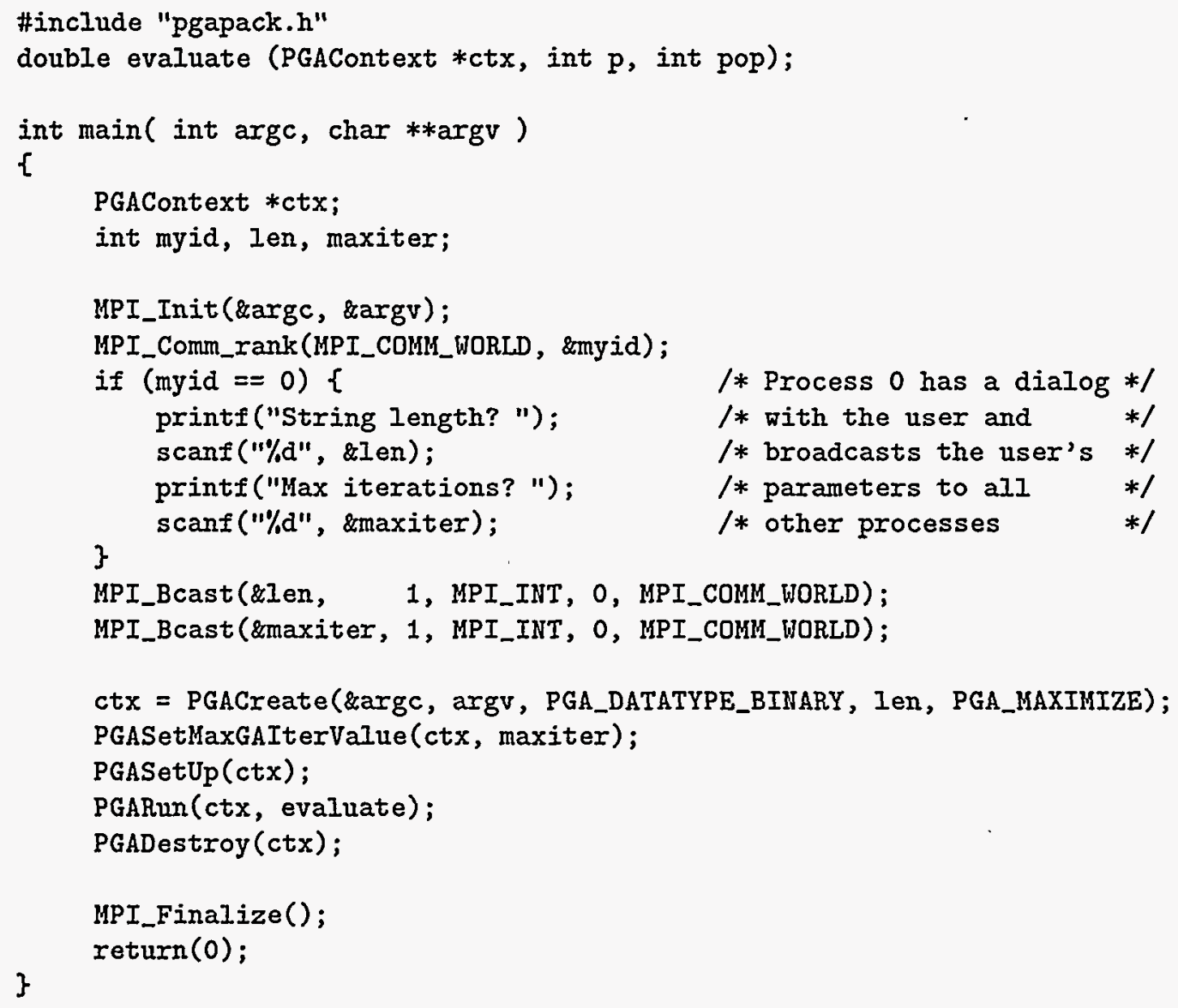

Figure 3.4: PGAPack Maxbit Example in $\mathrm{C}$ with $\mathrm{I} / \mathrm{O}$ 


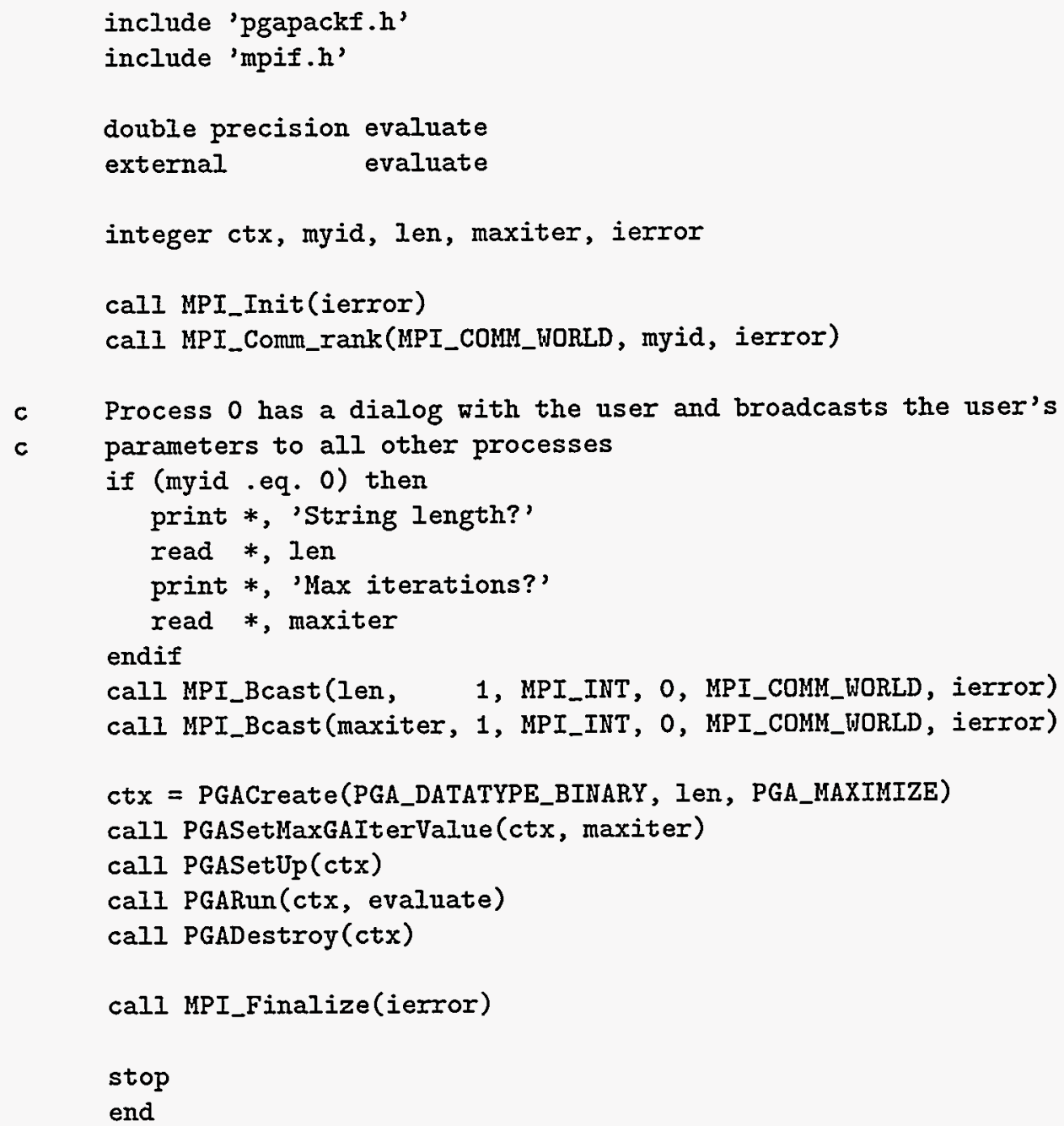

Figure 3.5: PGAPack Maxbit Example in Fortran with I/O

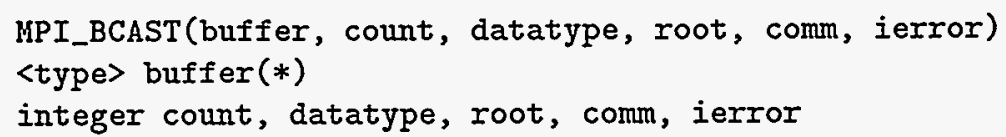

MPI Bcast will result in every process in communicator comm receiving a copy of the contents of *buf/buffer. The other parameters are the number of items (count), the datatype (datatype), which may be one of MPI_DOUBLE, MPI_INT, MPI_CHAR, MPI_UNSIGNED, or MPI_LONG; the rank of the process with the original copy (root); the MPI communicator (comm); and, for Fortran, a variable to handle an error return code (ierror).

\subsection{Compiling, Linking, and Execution}

When PGAPack was installed, the makefiles in the ./examples/c and ./examples/fortran directories were correctly configured for the machine PGAPack was installed on using the version of MPI specified (if any). To run your own programs, it is best to copy the appropriate makefile ( $\mathrm{C}$ or Fortran) to your directory and modify it to use your source code files. The makefile will compile your source code files, link in the PGAPack library (and MPI library if a parallel version of PGAPack was built), and build your executable.

How you execute your program will depend on whether a sequential or parallel version of PGAPack was 
built, the MPI implementation used and the machine you are running on. If a sequential version of PGAPack was built (i.e., one where the user did not supply a version of MPI), the executable maxbit can be executed on a uniprocessor Unix system by typing maxbit. If the MPICH implementation of MPI was used, it may be executed (using four processes) by mpirun maxbit -np 4. Appendix D contains some examples. 


\section{Part II}

\section{Users Guide}




\section{Chapter 4}

\section{The Structure of PGAPack}

This chapter provides a general overview of the structure of PGAPack.

\subsection{Native Data Types}

PGAPack is a data-structure-neutral library. By this we mean that a data-hiding capability provides the full functionality of the library to the user, in a transparent manner, irrespective of the data type used. PGAPack supports four native data types: binary-valued, integer-valued, real-valued, and character-valued strings. In addition, PGAPack is designed to be easily extended to support other data types (see Chapter 7).

The binary (or bit) data type (i.e., $|1| 0|1| 1 \mid$ ) is the traditional GA coding. The bits may either be interpreted literally or decoded into integer or real values by using either binary coded decimal or binary-reflected Gray codes. In PGAPack the binary data type is implemented by using each distinct bit in a computer word as a gene, making the software very memory-efficient. The integer-valued data type (i.e., $|3| 9|2| 4 \mid$ ) is often used in routing and scheduling problems. The real-valued data type (i.e., $|4.2| 7.1|-6.3| 0.8 \mid$ ) is useful in numerical optimization applications. The character-valued data type (i.e., $|\mathrm{h}| \mathrm{e}|\mathrm{I}| \mathrm{I}|\mathrm{o}| \mathrm{w}|\mathrm{o}| \mathrm{r}|\mathrm{I}| \mathrm{d} \mid$ is useful for symbolic applications.

\subsection{Context Variable}

In PGAPack the context variable is the data structure that provides the data hiding capability. The context variable is a pointer to a $C$ language structure, which is itself a collection of other structures. These (sub)structures contain all the information necessary to run the genetic algorithm, including data type specified, parameter values, which functions to call, operating system parameters, debugging flags, initialization choices, and internal scratch arrays. By hiding the actual data type selected and specific functions that operate on that data type in the context variable, user-level functions in PGAPack can be called independent of the data type.

Almost all fields in the context variable have default values. However, the user can set values in the context variable by using the PGASet family of function calls. The values of fields in the context variable may be read with the PGAGet family of function calls.

\subsection{Levels of Usage Available}

PGAPack provides multiple levels of control to support the requirements of different users. At the simplest level, the genetic algorithm "machinery" is encapsulated within the PGARun function, and the user need specify only three parameters: the data type, the string length, and the direction of optimization. All other parameters have default values. At the next level, the user calls the data-structure-neutral functions explicitly (e.g., PGASelect, PGACrossover, PGAMutation). This mode is useful when the user wishes more explicit control over the steps of the genetic algorithm or wishes to hybridize the genetic algorithm with a 
hill-climbing heuristic. At the third level, the user can customize the genetic algorithm by supplying his or her own function(s) to provide a particular operator(s) while still using one of the native data types. Finally, the user can define his or her own datatype, write the data-structure-specific low-level GA functions for the datatype (i.e., crossover, mutation, etc.), and have the data-structure-specific functions executed by the high-level data-structure-neutral PGAPack functions.

\subsection{Function Call-Based Library}

All the access to, and functionality of, the PGAPack library is providéd through function calls.

- The PGASet family of functions sets parameter values, allele values, and specifies which GA operators to use. For example, PGASetPopSize (ctx,500) sets the GA population size to 500 .

- The PGAGet family of functions returns the values of fields in the context variable and allele values in the string. For example, bit $=$ PGAGetBinaryAllele $(c t x, p, p o p, i)$ returns the value of the ith bit in string $P$ in population pop into bit.

- The simplest level of usage is provided by the PGARun function. This function will run the genetic algorithm by using any nondefault values specified by the user and default values for everything else.

- The next level of usage is provided by the data-structure-neutral functions, which the user can call to have more control over the specific steps of the genetic algorithm. Some of these functions are PGASelect, PGACrossover, PGAMutate, PGAEvaluate, and PGAFitness.

- The data-structure-specific functions deal directly with native data types. In general, the user never calls these functions directly.

- System calls in PGAPack provide miscellaneous functionality, including debugging, random number generation, output control, and error reporting.

\subsection{Header File and Symbolic Constants}

The PGAPack header file contains symbolic constants and type definitions for all functions and should be included in any file (or function or subroutine in Fortran) that calls a PGAPack function. For example, PGA_CROSSOVER_UNIFORM is a symbolic constant that is used as an argument to the function PGASetCrossoverType to specify uniform crossover. In $C$ the header file is pgapack.h. In Fortran it is pgapackf.h

\subsection{Evaluation Function}

PGAPack requires that the user supply a function that returns an evaluation of a string that it will map to a fitness value. This function is called whenever a string evaluation is required. The calling sequence and return value of the function must follow the format discussed in Section 5.9.

\subsection{Parallelism}

PGAPack can be run on both sequential computers (uniprocessors) and parallel computers (multiprocessors, multicomputers, and workstation networks). The parallel programming model used is message passing, in particular the single program, single data (SPMD) model. PGAPack version 1.0 supports sequential and parallel implementations of the global population model (see Chapter 10). 


\subsection{Implementation}

PGAPack is written in ANSI C. A set of interface functions allows most user-level PGAPack functions to be called from Fortran. All message-passing calls follow the Message Passing Interface (MPI) standard $[5,6]$. Nonoperative versions of the basic MPI functions used in the examples are supplied if the user does not provide an MPI implementation for their machine. These routines simply return and provide no parallel functionality. Their purpose is to allow the PGAPack library to be built in the absence of an MPI implementation.

Most low-level internal functions in PGAPack are data-structure specific and use addresses and/or offsets of the population data structures. The user-level routines, however, provide the abstractions of data-structure neutrality and an integer indexing scheme for access to population data structures. 


\section{Chapter 5}

\section{Basic Usage}

As the examples in Chapter 3 show, a PGAPack program can be written with just four function calls and a string evaluation function. This basic usage is discussed further in Section 5.1. Sections 5.3-5.12 explain options available in PGAPack. Section 5.13 discusses PGAPack command line arguments.

\subsection{Required Functions}

Any file (or function or subroutine in Fortran) that uses a PGAPack function must include the PGAPack header file. In $\mathrm{C}$ this file is pgapack.h. In Fortran this file is pgapackf.h. The first PGAPack call made is always to PGACreate. In $\mathrm{C}$ this call looks like

PGAContext *ctx;

ctx = PGACreate (2argc, argv, datatype, len, maxormin);

PGACreate allocates space for the context variable, ctx (Section 4.2), and returns its address. argc and argv are the standard list of arguments to a $C$ program. datatype must be one of PGA_DATATYPE_BINARY, PGA_DATATYPE_INTEGER, PGA_DATATYPE_REAL, Or PGA_DATATYPE_CHARACTER to specify strings consisting of binary-valued, integer-valued, real-valued, or character-valued strings, respectively. len is the length of the string (i.e., the number of genes). maxormin must be PGA_MAXIMIZE or PGA_MINIMIZE to indicate whether the user's problem is maximization or minimization, respectively.

In Fortran the call to PGACreate is

integer ctx

ctx = PGACreate (datatype, len, maxormin)

where datatype, Ien, and maxormin are the same as for C programs. After the PGACreate call, the user may optionally set nondefault values. These are then followed by a call to PGASetUp to initialize to default values all options, parameters, and operators not explicitly specified by the user. For example,

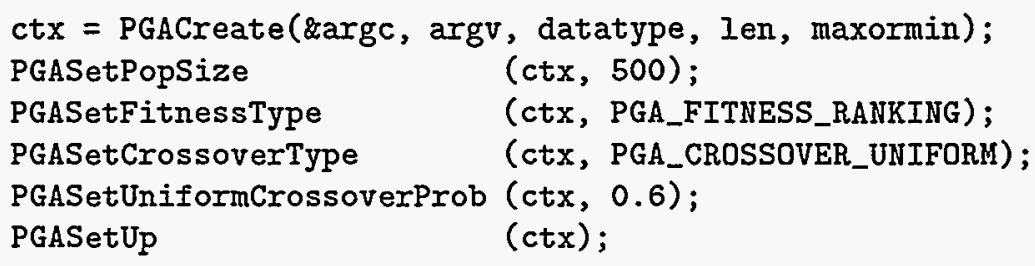

will change the default values for the population size, the mapping of the user's evaluation to a fitness value, and the crossover type. All PGASet calls should be made after PGACreate has been called, but before PGASetUp has been called; all such calls are optional. Note also that all PGAPack functions other than PGACreate take the context variable as their first argument.

The PGARun function executes the genetic algorithm. Its second argument is the name of a user-supplied evaluation function that returns a double (double precision in Fortran) value that is the user's evaluation of an individual string. In $\mathrm{C}$ the prototype for this function looks like 
double evaluate (PGAContext $*$ ctx, int $\mathrm{p}$, int $\mathrm{pop}$ );

and in Fortran

double precision function evaluate (ctx, p, pop)

integer ctx, $p$, pop

The user must write the evaluation function, and it must have the calling sequence shown above and discussed further in Section 5.9. After PGARun terminates, PGADestroy is called to release all memory allocated by PGAPack. 1

Except for writing an evaluation function (Section 5.9) the information contained in rest of this chapter is optional-defaults will be set for all other GA parameters. We do note, however, that the defaults used are the result of informal testing and results reported in the GA literature. They are by no means optimal, and additional experimentation with other values may well yield better performance on any given problem.

\subsection{Population Replacement}

Two population replacement schemes are common in the literature. The first, the generational replacement genetic algorithm (GRGA), replaces the entire population each generation and is the traditional genetic algorithm [7]. The second, the steady-state genetic algorithm (SSGA), typically replaces only a few strings each generation and is a more recent development $[9,10,11]$. PGAPack supports both GRGA and SSGA and variants in between via parameterized population replacement. For example, the PGASet calls

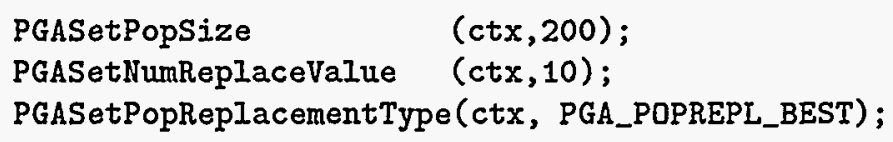

specify that each generation a new population is created consisting of ten strings created via recombination, and the 190 most fit strings from the old population. The 190 strings can also be selected randomly, with or without replacement, by setting the second argument of PGASetPopReplacementType to PGA_POPREPL_RANDOM_REP or PGA_POPREPL_RANDOM_NOREP, respectively.

By default, the number of new strings created each generation is 10 percent of the population size (an SSGA population replacement strategy). A GRGA can be implemented by setting PGASetNumReplaceValue to the population size (the default population size is 100). Setting PGASetNumReplaceValue to one less than the population size will result in an elitist GRGA, where the most fit string is always copied to the new population (since PGA_POPREPL_BEST is the default population replacement strategy).

Traditionally, strings created through recombination first undergo crossover and then mutation. Some practitioners [3] have argued that these two operators should be separate. By default, PGAPack applies mutation only to strings that did not undergo crossover. This is equivalent to setting PGASetKutationOrCrossoverFlag (ctx, PGA_RUE). To have strings undergo both crossover and mutation, one should use PGASetMutationAndCrossoverFlag (ctx,PGA_TRUE).

By default, PGAPack allows duplicate strings in the population. Some practitioners advocate not allowing duplicate strings in the population in order to maintain diversity. The function call PGASetNoDuplicatesFlag (ctx, PGA_TRUE) will not allow duplicate strings in the population: It repeatedly applies the mutation operator (with an increasing mutation rate) to a duplicate string until it no longer matches any string in the new population. If the mutation rate exceeds 1.0 , however, the duplicate string will be allowed in the population, and a warning message will be issued.

Figure 5.1 shows the generic population replacement scheme in PGAPack. Both populations $k$ and $k+1$ are of fixed size (the value returned by PGAGetPopSize). First, PGAGetPopSize - PGAGetNumReplaceValue strings are copied over directly from generation $k$. The way the strings are chosen, the most fit, or randomly with or without replacement, depends on the value set by PGASetPopReplacementType. The remaining PGAGetNumReplaceValue strings are created by crossover and mutation.

\footnotetext{
${ }^{1}$ PGADestroy will also call MPI finalize, if MPI was started by PGACreate.
} 

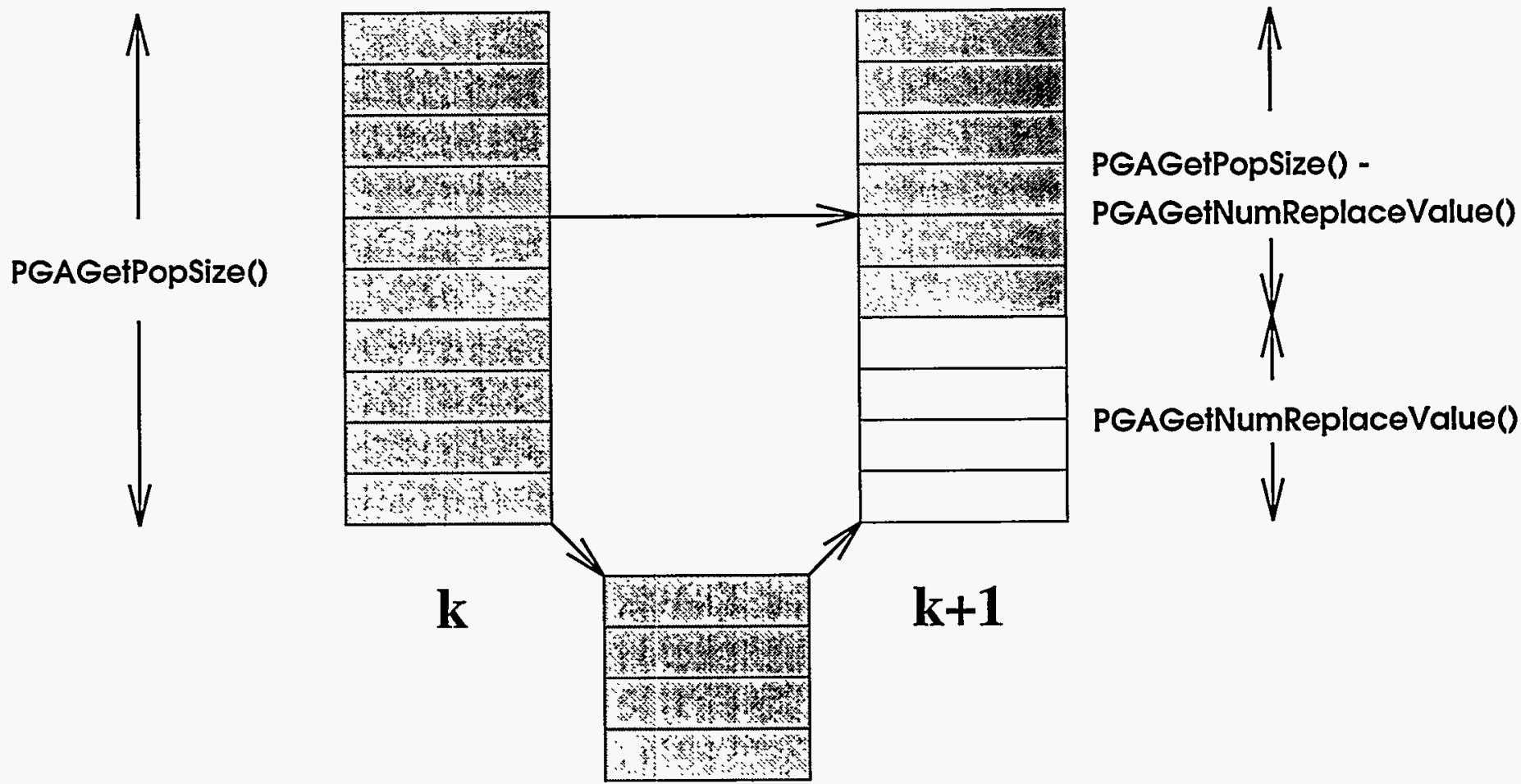

\section{temp}

Figure 5.1: Population Replacement 


\subsection{Stopping Criteria}

PGAPack terminates when at least one of the stopping rule(s) specified has been met. The three stopping rules are (1) iteration limit exceeded, (2) population too similar, and (3) no change in the best solution found in a given number of iterations. The default is to stop when the iteration limit (by default, 1000 iterations) is reached.

The choice of stopping rule is set by PGASetStoppingRuleType. For example, PGASetStoppingRuleType (ctx,PGA_STOP_HAXITER) is the default. Other choices are PGA_STOP_TOOSIMILAR and PGA_STOP_NOCHANGE for population too similar and no change in the best solution found, respectively. PGASetStoppingRuleType may be called more than once. The different stopping rules specified are ored together.

If PGA_STOP_MAXITER is one of the stopping rules, PGASetMaxGAIterValue(ctx,500) will change the maximum iteration limit to 500. If PGA_STOP_NOCHANGE is one of the stopping rules, PGASetMaxNoChangeValue (ctx,50) will change from 100 (the default) to 50 the maximum number of iterations in which no change in the best evaluation is allowed before the GA stops. If PGA_STOP_TOOSIMILAR is one of the stopping rules, PGASetMaxSimilarityValue (ctx,99) will change from 95 to 99 the percentage of the population allowed to have the same evaluation function value before the GA stops.

\subsection{Initialization}

Strings are either initialized randomly (the default), or set to zero. The choice is specified by setting the second argument of PGASetRandomInitFlag to either PGA_TRUE or PGA_FALSE, respectively. Random initialization depends on the datatype.

If binary-valued strings are used, each gene is set to 1 or 0 with an equal probability. To set the probability of randomly setting a bit to 1 to 0.3 , use PGASetBinaryInitProb(ctx, 0.3$)$.

For integer-valued strings, the default is to set the strings to a permutation on a range of integers. The default range is $[0, L-1]$, where $L$ is the string length. PGASetIntegerInitPermute(ctx, 500, 599) will set the permutation range to $[500,599]$. The length of the range must be the same as the string length.

Alternatively, PGASetIntegerInitRange will set each gene to a random value sélected uniformly from a specified range. For example, the code

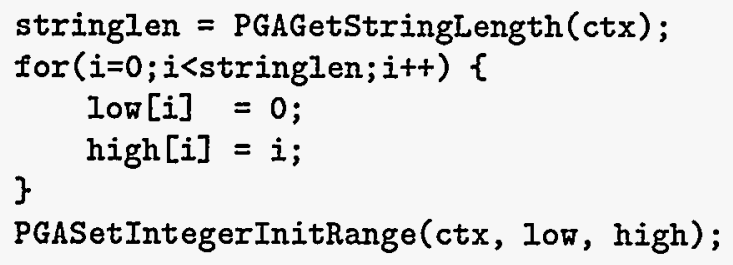

will select a value for gene $i$ uniformly randomly from the interval $[0, i]$.

If real-valued strings are used, the alleles are set to a value selected uniformly randomly from a specified interval. The interval may be specified with either the PGASetRealInitRange or PGASetRealInitPercent functions. For example, the code

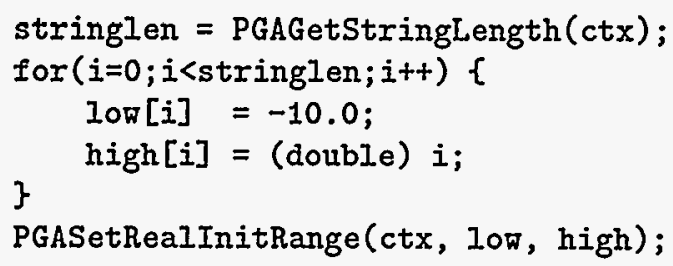

will select a value for allele $i$ uniformly randomly from the interval $[-10.0, i]$. This is the default strategy for initializing real-valued strings. The default interval is $[0,1.0]$.

PGASetRealInitPercent specifies the interval with a median value and percent offset. For example,

stringlen $=$ PGAGetStringLength (ctx);

for $(i=1 ; i<=$ stringlen; $i+t)\{$

median $[i]=($ double $) i$; 


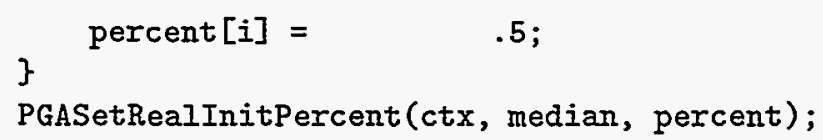

will select a value for allele $i$ uniformly randomly from the increasing intervals $\left[\frac{1}{2} i, \frac{3}{2} i\right]$. Note that if the median value is zero for some $i$, than an interval of $[0,0]$ will be defined.

If character-valued strings are used, PGASetCharacterInitType (ctx, PGA_CINIT_UPPER) will set the allele values to uppercase alphabetic characters chosen uniformly randomly. Other options are PGA_CINIT LOWER for lower case letters only (the default) and PGA_CINIT_MIXED for mixed case letters, respectively.

\subsection{Selection}

The selection phase allocates reproductive trials to strings on the basis of their fitness. PGAPack supports four selection schemes: proportional selection, stochastic universal selection, binary tournament selection, and probabilistic binary tournament selection. The choice may be specified by setting the second argument of PGASetSelectType to one of PGA_SELECT_PROPORTIONAL, PGA_SELECT_SUS, PGA_SELECT_TOURNAMENT, and PGA_SELECT_PTOURNAMENT for proportional, stochastic universal, tournament, and probabilistic tournament selection, respectively. The default is tournament selection. For probabilistic tournament selection, the default probability that the string that wins the tournament is selected is 0.6 . It may be set to 0.8 , for example, with PGASetPTournamentProb(ctx, 0.8).

\subsection{Crossover}

The crossover operator takes bits from each parent string and combines them to create child strings. The type of crossover may be specified by setting PGASetCrossoverType to PGA_CROSSOVER_ONEPT, PGA_CROSSOVER_TWOPT, or PGA_CROSSOVER_UNIFORM for one-point, two-point, or uniform crossover, respectively. The default is twopoint crossover. By default the crossover rate is 0.85 . It may be set to 0.6 by PGASetCrossoverProb(ctx, 0.6 ), for example.

Uniform crossover is parameterized by $p_{u}$, the probability of swapping two parent bit values [8]. By default, $p_{u}=0.5$. The function call PGASetUniformCrossoverProb(ctx, 0.7 ) will set $p_{u}=0.7$.

\subsection{Mutation}

The mutation rate is the probability that a gene will undergo mutation. The mutation rate is independent of the datatype used. The default mutation rate is the reciprocal of the string length. The function call PGASetMutationProb(ctx,.001) will set the mutation rate to .001 .

The type of mutation depends on the data type. For binary-valued strings, mutation is a bit complement operation For character-valued strings, mutation replaces one alphabetic character with another chosen uniformly randomly. The alphabetic characters will be lower, upper, or mixed case depending on how the strings were initialized.

For integer-valued strings, if the strings were initialized to a permutation and gene $i$ is to be mutated, the default mutation operator swaps gene $i$ with a randomly selected gene. If the strings were initialized to a random value from a specified range and gene $i$ is to be mutated, by default gene $i$ will be replaced by a value selected uniformly random from the initialization range.

The mutation operator for integer-valued strings may be changed irrespective of how the strings were initialized. If PGASetMutationType is set to PGA_MUTATION_RANGE, gene $i$ will be replaced with a value selected uniformly randomly from the initialization range. If the strings were initialized to a permutation, the minimum and maximum values of the permutation define the range. If PGASetMutationType is set to PGA_MUTATION_PERMUTE, gene $i$ will be swapped with a randomly selected gene. If PGASetMutationType is set to PGA MUTATION_CONSTANT, a constant integer value (by default one) will be added (subtracted) to (from) the existing allele value. The constant value may be set to 34 , for example, with PGASetMutationIntegerValue (ctx,34). 
Three of the four real-valued mutation operators are of the form $v \leftarrow v \pm p \times v$, where $v$ is the existing allele value. They vary by how $p$ is selected. First, if PGASetHutationType is set to PGA MUTATION_CONSTANT, $p$ is the constant value 0.01 . It may be set to .02 , for example, with PGASetMutationRealValue(ctx, .02). Second, if PGASetMutationType is set to PGA.MUTATION_UNIFORM, $p$ is selected uniformly from the interval $(0,1)$. To select $p$ uniformly from the interval $(0,1)$ set PGASetMutationRealValue $(\operatorname{ctx}, 1)$. Third, if PGASetMutationType is set to PGA_MUTATION_GAUSSIAN, $p$ is selected from a Gaussian distribution (this is the default real-valued mutation operator) with mean 0 and standard deviation 0.1 . To select $p$ from a Gaussian distribution with mean 0 and standard deviation 0.5 set PGASetMutationRealValue(ctx, .5). Finally, if PGASetlutationType is set to PGA MUTATION_RANGE, gene $i$ will be replaced with a value selected uniformly random from the initialization range of that gene.

Some of the integer- and real-valued mutation operators may generate allele values outside the initialization range of that gene. If this happens, by default, the allele value will be reset to the lower (upper) value of the initialization range for that gene. By setting PGASetMutationBoundedFlag(ctx, PGAFALSE) the allele values will not be reset if they fall outside of the initialization range.

\subsection{Restart}

The restart operator reseeds a population from the best string. It does so by seeding the new population with the best string and generating the remainder of the population as mutated variants of the best string.

By default the restart operator is not invoked. Setting PGASetRestartFlag(ctx, PGATRUE) will cause the restart operator to be invoked. By default PGAPack will restart every 50 iterations. PGASetRestartFrequencyValue $(\operatorname{ctx}, 100)$ will restart every 100 iterations instead. When creating the new strings from the best string an individual allele undergoes mutation with probability 0.5 . This can be changed to 0.9 with the function call PGASetRestartAlleleChangeProb (ctx, 0:9).

For binary-valued strings the bits are complemented. For integer- and real-valued strings the amount to change is set with PGASetMutationIntegerValue and PGASetMutationRealValue, respectively. Charactervalued strings are changed according to the rules in Section 5.7 for mutating character strings.

\subsection{String Evaluation and Fitness}

In a genetic algorithm each string is assigned a nonnegative, real-valued fitness. This is a measure, relative to the rest of the population, of how well that string satisfies a problem-specific metric. In PGAPack calculating a string's fitness is a two-step process. First, the user supplies a real-valued evaluation (sometimes called the raw fitness) of each string. Second, this value is mapped to a fitness value.

It is the user's responsibility to supply a function to evaluate an individual string. As discussed in Section 5.1, the name of this function is specified as the second argument to PGARun. The calling sequence for this function (which we call evaluate in the rest of this section, but may have any name) must follow the format given here. In $\mathrm{C}$ the format is

double evaluate (PGAContext *ctx, int $p$, int pop);

and in Fortran

double precision function evaluate (ctx, p, pop)

integer ctx, $\mathrm{p}$, pop

The function evaluate will be called by PGARun whenever a string evaluation is required. $p$ is the index of the string in population pop that will be evaluated. The correct values of $p$ and pop will be passed to the evaluation function by PGARun. (If PGARun is not used, PGAEvaluate must be. See Chapter 6.) A's shown below, $p$ and pop are used for reading (and sometimes writing) allele values Sample evaluation functions are shown in Figures 3.1 and 3.2, and online in the ./examples directory.

Traditionally, genetic algorithms assume fitness values are nonnegative and monotonically increasing the more fit a string is. The user's evaluation of a string, however, may reflect a minimization problem and/or be negative. Therefore, the user's evaluation value is mapped to a nonnegative and monotonically increasing 
fitness value. First, all evaluations are mapped to positive values (if any were negative). Next, these values are translated to a maximization problem (if the direction of optimization specified was minimization). Finally, these values are mapped to a fitness value by using the identity (the default), linear ranking, or linear normalization, The choice of fitness mapping may be set with the function PGASetFitnessType. The second argument must be one of PGA_FITNESS_RAW, PGAFITNESS_RANKING, or PGA_FITNESS_NORMAL, for the identity, linear ranking, or linear normalization, respectively.

A linear rank fitness function $[2,10]$ is given by

$$
\operatorname{Min}+(\operatorname{Max}-\operatorname{Min}) \frac{\operatorname{rank}(\mathrm{p})-1}{N-1},
$$

where $\operatorname{rank}(\mathrm{p})$ is the index of string $\mathrm{p}$ in a list sorted in order of decreasing evaluation function value, and $N$ is the population size. Ranking requires that $1 \leq \operatorname{Max} \leq 2$, and $M i n+\operatorname{Max}=2$. The default value for $M a x$ is 1.2. It may be set to 1.1 with PGASetMaxFitnessRank(ctx,1.1).

In linear normalization the fitness function is given by

$$
K-(\operatorname{rank}(\mathrm{p}) * C),
$$

where $K$ and $C$ are the constants $\sigma * N$ and $\sigma$, where $\sigma$ is the standard deviation of the user's evaluation function values after they have been transformed to positive values for a maximization problem.

If the direction of optimization is minimization, the values are remapped for maximization. The function call PGASetFitnessMinType(ctx,PGA_FITNESSMIN_CMAX) will remap by subtracting the worst evaluation value from each evaluation value (this is the default). The worst evaluation value is multiplied by 1.01 before the subtraction so that the worst string has a nonzero fitness. The function call PGASetFitnessCmaxValue (ctx, 1.2) will change the multiplier to 1.2 Alternatively, if PGA_FITNESSMIN_RECIPROCAL is specified the remapping is done by using the reciprocal of the evaluation function.

\subsection{Accessing Allele Values}

For each of the native data types, PGAPack provides a matched pair of functions that allow the user to read or write (change) any allele value. If the data type is PGA_DATATYPE_BINARY

int bit;

bit $=$ PGAGetBinaryAllele (ctx, p, pop, i);

will assign to bit the binary value of the ith gene in string $\mathrm{p}$ in population pop. To set the ith gene in string $\mathrm{p}$ in population pop to 1 , use

PGASetBinaryAllele(ctx, p, pop, $i, 1)$;

If the data type is PGADATATYPE_INTEGER

int $\mathrm{k}$;

$k=$ PGAGetIntegerAllele (ctx, p, pop, $i)$;

will assign to $\mathrm{k}$ the integer value of the $i$ th gene in string $\mathrm{p}$ in population pop. To set the $i$ th gene in string $p$ in population pop to 34 , use

PGASetIntegerAllele(ctx, p, pop, i, 1, 34);

If the data type is PGA_DATATYPE_REAL

double $x$;

$x=$ PGAGetRealAllele (ctx, p, pop, i);

will assign to $x$ the real value of the ith gene in string $\mathrm{p}$ in population pop. To set the ith gene in string $\mathrm{p}$ in population pop to 123.456 , use

PGASetRealAIlele(ctx, p, pop, i, 1, 123.456); 
If the data type is PGA_DATATYPE_CHARACTER

char c;

$c=$ PGAGetCharacterAllele (ctx, p, pop, i);

will assign to $c$ the character value of the ith gene in string $\mathrm{p}$ in population pop. To set the $i$ th gene in string $\mathrm{P}$ in population pop to "Z", use

PGASetCharacterAllele(ctx, p, pop, i, 1, ' $\left.Z^{\prime}\right)$;

\subsubsection{Representing an Integer with a Binary String}

A binary string may be used to represent an integer by decoding the bits into an integer value. In a binary coded decimal (BCD) representation, a binary string is decoded into an integer $k \in\left[0,2^{N}-1\right]$ according to

$$
k=\sum_{i=1}^{N} b_{i} 2^{i-1},
$$

where $N$ is the string length, and $b_{i}$ the value of the $i$ th bit. For example, to decode the integer $\mathrm{k}$ from the ten bits in bit positions $20-29$, use

int $\mathbf{k}$

$\mathrm{k}=$ PGAGetIntegerFromBinary (ctx,p,pop, 20,29);

The function PGAEncodeIntegerAsBinary will encode an integer as a binary string. For example, to encode the integer 564 as a 12-bit binary string ${ }^{2}$ in the substring defined by bits $12-23$, use

PGAEncodeIntegerAsBinary (ctx,p,pop, $12,23,564)$;

In a BCD representation, two numbers that are contiguous in their decimal representations may be far from each other in their binary representations. For example, 7 and 8 are consecutive integers, yet their 4-bit binary representations, 0111 and 1000, differ in the maximum number of bit positions. ${ }^{3}$ Gray codes define a different mapping of binary strings to integer values from that given by Eq. (5.3) and may alternatively be used for representing integer (or real, see below) values in a binary string. The second and third columns in Table 5.1 show how the integers 0-7 are mapped to Eq. (5.3) and to the binary reflected Gray code (the most commonly used Gray code sequence), respectively. In the binary reflected Gray code sequence, the binary representations of consecutive integers differ by only one bit (a Hamming distance of one).

To decode the integer $\mathrm{k}$ from a binary reflected Gray code interpretation of the binary string, use

$\mathbf{k}=$ PGAGetIntegerFromGrayCode (ctx,p,pop, 20, 29);

To encode 564 as a 12-bit binary string in the substring defined by bits 12-23 using a Gray code, use

PGAEncodeIntegerAsGaryCode(ctx,p,pop, 12, 23, 564);

\subsubsection{Representing a Real Value with a Binary String}

A binary string may also be used to represent a real value. The decoding of a binary string to a real-value is a two-step process. First, the binary string is decoded into an integer as described in Section 5.10.1. Next, the integer is mapped from the discrete interval $\left[0,2^{N}-1\right]$ to the real interval $[L, U]$ by using the formula

$$
x=(k-a) \times(U-L) /(b-a)+L
$$

(and generalizing $\left[0,2^{N}-1\right]$ to $\left.[a, b]\right)$. For example, to decode the double $x$ from the 20 bits given by the binary string stored in bit positions $10-29$ onto the interval $[-10.0,20.0]$, use

\footnotetext{
${ }^{2}$ Even though only ten bits are necessary to encode 564 , the user may want to allow the GA any value between $[0,4095]$, hence the twelve bits.

${ }^{3}$ Technically, this is known as a Hamming cliff.
} 
Table 5.1: Binary and Gray Codes

\begin{tabular}{|c|r|r|}
\hline \hline$k$ & Eq. (5.3) & Gray code \\
\hline 0 & 000 & 000 \\
1 & 001 & 001 \\
2 & 010 & 011 \\
3 & 011 & 010 \\
4 & 100 & 110 \\
5 & 101 & 111 \\
6 & 110 & 101 \\
7 & 111 & 100 \\
\hline
\end{tabular}

$x=$ PGAGetRealFromBinary $(c t x, p, p \circ p, 10,29,-10.0,20.0) ;$

To encode -18.3 on the interval $[-50.0,50.0]$ using a 20 -bit BCD binary string, use

PGAEncodeRealAsBinary (ctx, p, pop, $0,19,-50.0,50.0,-18.3)$;

The functions PGAGetRealFromGrayCode and PGAEncodeRealAsGrayCode provide similar functionality for Gray-coded strings.

\subsubsection{Example}

As an example, suppose the user has a real-valued function $f$ of three real variables $x_{1}, x_{2}$, and $x_{3}$. Further, the variables are constrained as follows.

$$
\begin{gathered}
-10 \leq x_{1} \leq 0 \\
0 \leq x_{2} \leq 10 \\
-10 \leq x_{3} \leq 10
\end{gathered}
$$

The user wishes to use 10 bits for the binary representation of $x_{1}$ and $x_{2}$, and 20 bits for the binary representation of $x_{3}$ (perhaps for higher accuracy), and a Gray code encoding. This may be done as follows.

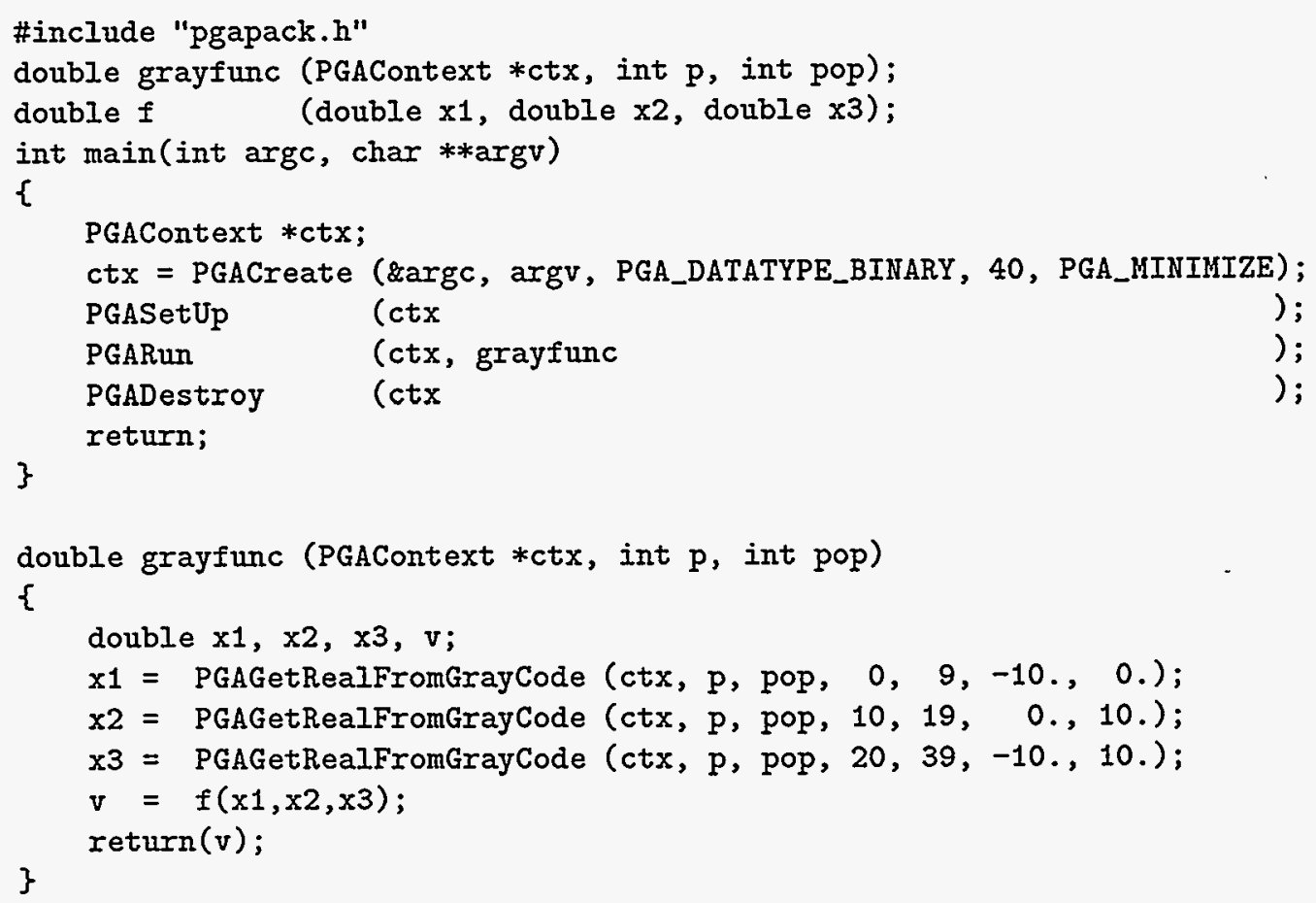


In Fortran, the bit indices would be 1-10, 11-20, and 21-40, respectively. The number of bits allocated for the binary representation determines the accuracy with which the real value can be calculated. Note in this example the function $f$ need not be modified; the function grayfunc is used as a "wrapper" to get variable values out of the GA and return the value calculated by $f$.

\subsection{Report Options}

PGASetPrintFrequencyValue (ctx, 40 ) will print population statistics every 40 iterations. The default is every ten iterations. The best evaluation is always printed. To print additional statistics, set the second argument of the function PGASetPrint0ptions to PGA_REPORT_ONLINE, PGA_REPORT_OFFLINE, PGA_REPORT_WORST, PGA_REPORT_AVERAGE, PGA_REPORT_HAMMING, OI PGA_REPORT_STRING to print the online analysis, offline analysis, worst evaluation, average evaluation, Hamming distance, or string itself, respectively. PGASetPrintoptions may be called multiple times to specify multiple print options.

\subsection{Utility Functions}

\subsubsection{Random Numbers}

By default, PGAPack will seed its random number generator by using a value from the system clock. Therefore, each time PGAPack is run, a unique sequence of random numbers will be used. For debugging or reproducibility purposes, however, the user may wish to use the same sequence of random numbers each time. This may be done using the function PGASetRandomSeed to initialize the random number generator with the same seed each time, for example, PGASetRandomSeed $(\operatorname{ctx}, 1)$.

PGARandom01 $(\operatorname{ctx}, 0)$ will return a random number generated uniformly on $[0,1]$. If the second argument is not 0 , it will be used to reseed the random number sequence. PGARandomflip flips a biased coin. For example, PGARandomFlip(ctx, .7) will return PGA_TRUE approximately $70 \%$ of the time. PGARandomInterval $(-10,30)$ will return an integer value generated uniformly on $[-10,30]$. PGARandomUniform (ctx,$-50 ., 50$.) will return a real value generated uniformly randomly on the interval $[-50,50]$. PGARandomGaussian (ctx, $0 ., 1$.) will return a real value generated from a Gaussian distribution with mean zero and standard deviation one.

\subsubsection{Print Functions}

PGAPrintPopulation (ctx, stdout,pop) will print the evaluation function value, fitness value, and string for each member of population pop to stdout. This function may not be called until after PGASetUp has been called. PGAPrintContextVariable(ctx,stdout) will print the value of all fields in the context variable to stdout. PGAPrintIndividual (ctx, stdout, $p$,pop) will print the evaluation function value, fitness value, and string of individual $p$ in population pop to stdout. PGAPrintString (ctx, stdout, $p, p o p$ ) will print the string of individual $p$ in population pop to stdout. PGAPrintVersionNumber(ct $x$ ) will print the PGAPack version number.

\subsubsection{Miscellaneous}

PGAGetGAIterValue(ctx) will return the current iteration of the GA. PGAGetBestIndex(ctx,pop) (PGAGetWorstIndex) will return the index of the most (least) fit member of population pop.

PGAUpdateOffline(ctx,pop) (PGAUpdateOnline) will update the offline (online) analysis based on the new generation's results. PGAHammingDistance (ctx, pop) returns a double, which is the average Hamming distance between'the binary strings in population pop. The function call

PGAError(ctx, "popindex=", PGA_FATAL, PGA_INT, (void *)\&popindex)

will print the message "popindex=-1" (assuming the value of popindex is -1 ) and then exit PGAPack. If the third argument had been PGA_WARNING instead, execution would have continued. In addition to PGA.INT, valid data types are PGA_DOUBLE, PGA_CHAR, and PGA_VOID. 


\subsection{Command-Line Arguments}

PGAPack provides several command-line arguments. These are only available to $\mathrm{C}$ programs, although in some cases both $\mathrm{C}$ and Fortran programs can achieve the equivalent functionality with function calls. For example, PGAUsage(ctx) provides the same functionality as the -pgahelp command line option. See Chapter 12 for the function call equivalents.

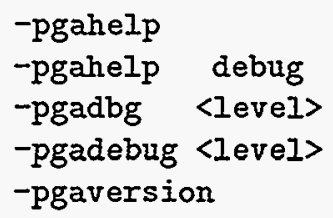

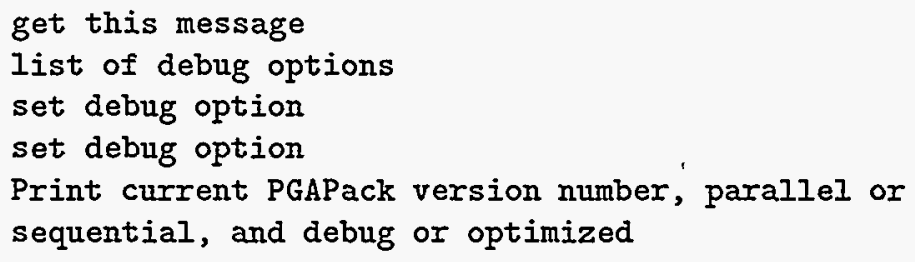




\section{Chapter 6}

\section{Explicit Usage}

This chapter discusses how the user may obtain greater control over the steps of the GA by not using the PGARun command, but instead calling the data-structure-neutral functions directly. One ramification of this is that the PGARun interface no longer masks some of the differences between parallel and sequential execution. The examples in this chapter are written for sequential execution only. Chapter 10 shows how they may be executed in parallel.

\subsection{Notation}

To understand the calling sequences of the functions discussed in this chapter, one must know of the existence of certain data structures and the user interface for accessing them. It is not necessary to know how these data structures are implemented, since that is hidden by the user interface to PGAPack.

PGAPack maintains two populations: an old one and a new one. The size of each population is the value returned by PGAGetPopSize. In addition, each population contains two temporary working locations. The string length is the value specified to PGACreate and returned by PGAGetStringLength.

Formally, string $p$ in population pop is referred to by the 2-tuple ( $p, p \circ p)$ and the value of gene $i$ in that string by the 3-tuple ( $i, p, p o p)$. In PGAPack, pop must be one of the two symbolic constants PGA_OLDPOP or PGA_NEWPOP to refer to the old or new population, respectively. At the end of each GA iteration, the function PGAUpdateGeneration makes sure these symbolic constants are remapped to the correct population. 'The string index $p$ must be either an integer between 0 and $P-1$ (or 1 and $P$ in Fortran) or one of the symbolic constants PGA_TEMP1 or PGA_TEMP2, to reference one of the two temporary locations, respectively.

\subsection{Simple Sequential Example}

The example in Figure 6.1 is a complete PGAPack program that does not use PGARun. It is an alternative way to write the main program for the Maxbit example of Section 3.1. We refer to it as a simple example because it uses PGARunMutationAndCrossover to encapsulate the recombination step. The PGACreate and PGASetUp functions were discussed in the last chapter. PGASetUp creates and randomly initializes the initial population. This population, referred to initially by the symbolic constant PGA_OLDPOP, is evaluated by the PGAEvaluate function. The third argument to PGAEvaluate is the name of the user's evaluation function. The function prototype for evaluate must be as shown in Figure 6.1 and discussed earlier in Sections 5.1 and 5.9. The PGAFitness function maps the user's evaluation function values into fitness values.

The while loop runs the genetic algorithm. PGADone returns PGA_TRUE if any of the specified stopping criteria have been met, otherwise PGAFALSE. PGASelect performs selection on population PGA_OLDPOP. PGARunMutationAndCrossover uses the selected strings to create the new population by applying the crossover and mutation operators. PGAEvaluate and PGAFitness evaluate and map to fitness values the newly created population. PGAUpdateGeneration updates the GA iteration count and resets several important internal arrays (don't forget to call it!). PGAPrintReport writes out genetic algorithm statistics 


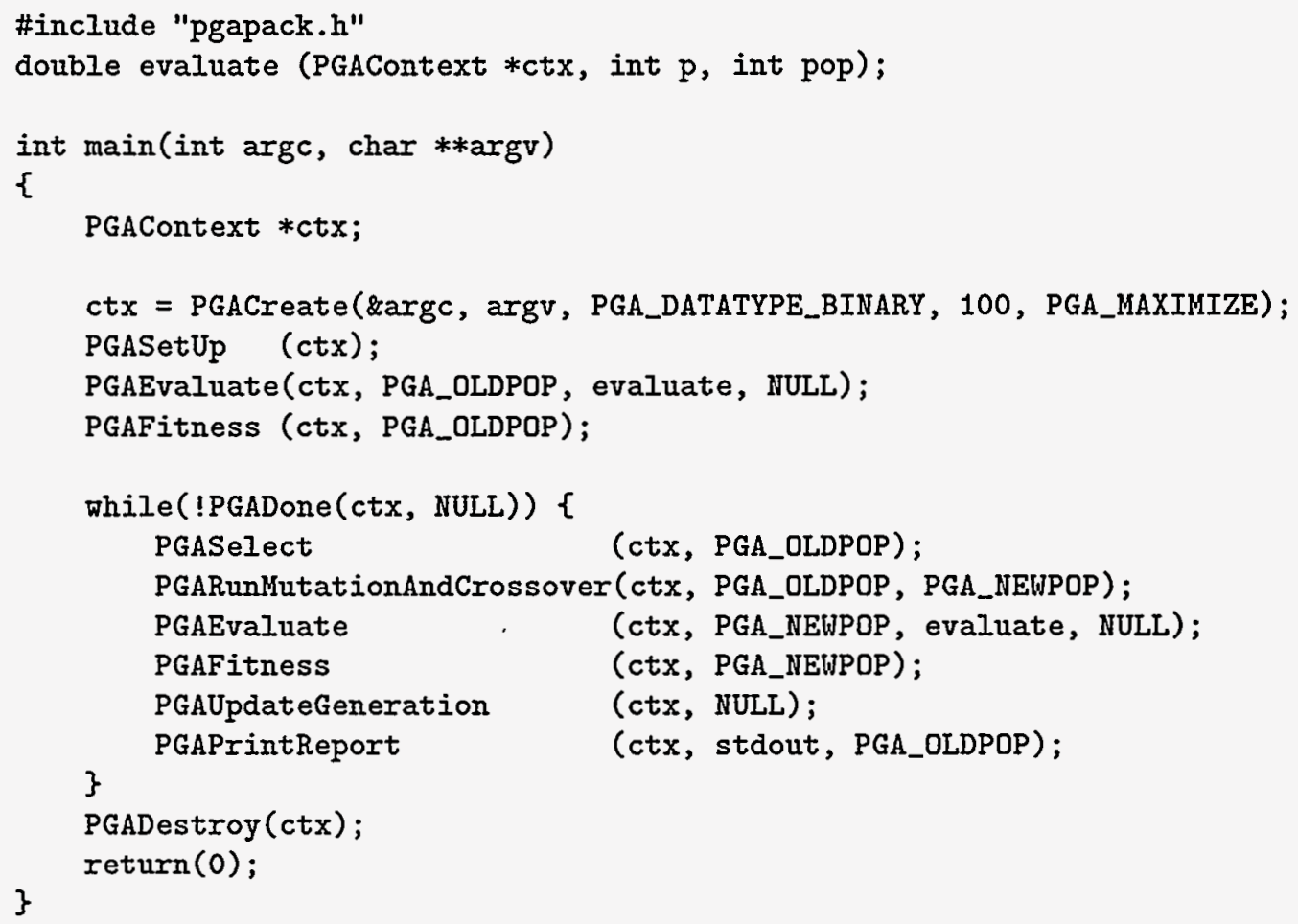

Figure 6.1: Simple Example of Explicit Usage

according to the report options specified. Note that the argument to PGAPrintReport is the old population, since after PGAUpdateGeneration is called, the newly created population is in PGA_OLDPOP. Finally, PGADestroy releases any memory allocated by PGAPack when execution is complete.

The functions PGADone, PGAUpdateGeneration, and PGAEvaluate take an MPI communicator (see Appendix $\mathrm{C}$ and Chapter 10) as an argument. For sequential execution the value NULL should be specified for this argument. A parallel, or sequential and parallel, version of this example is given in Section 10.2.

\subsection{Complex Example}

The primary difference between the "complex" example in Figure 6.2 and the "simple" example in Figure 6.1 is that the steps encapsulated by PGARunHutationAndCrossover have been written out explicitly. The function PGASortPop sorts a population according to the criteria specified by PGASetPopReplacementType (Section 5.2). The sorted indices are accessed via PGAGetSortedPopIndex. In the example, the five lines that follow PGASortPop copy the strings that are not created by recombination from the old population to the new population.

The while loop that follows creates the remainder of the new population. PGASelectllextIndex returns the indices of the strings selected by PGASelect. PGARandomFlip flips a coin biased by the crossover probability to determine whether the selected strings should undergo crossover and mutation or should be copied directly into the new population. PGACrossover uses the parent strings $m 1$ and $m 2$ from population PGA_OLDPOP to create two child strings in the temporary locations PGA_TEMP1 and PGA_TEMP2 in PGA_NEWPOP population.

PGAMutate mutates the child strings and PGACopyIndividual, then copies them into the new population. If the strings do not undergo crossover and mutation, they are copied into the new population unchanged. The rest of the steps are the same as those in Figure 6.1, except that for illustrative purposes we call PGAPrintReport before PGAUpdateGeneration. In that case we use population PGA_NEWPOP as the population pointer. 


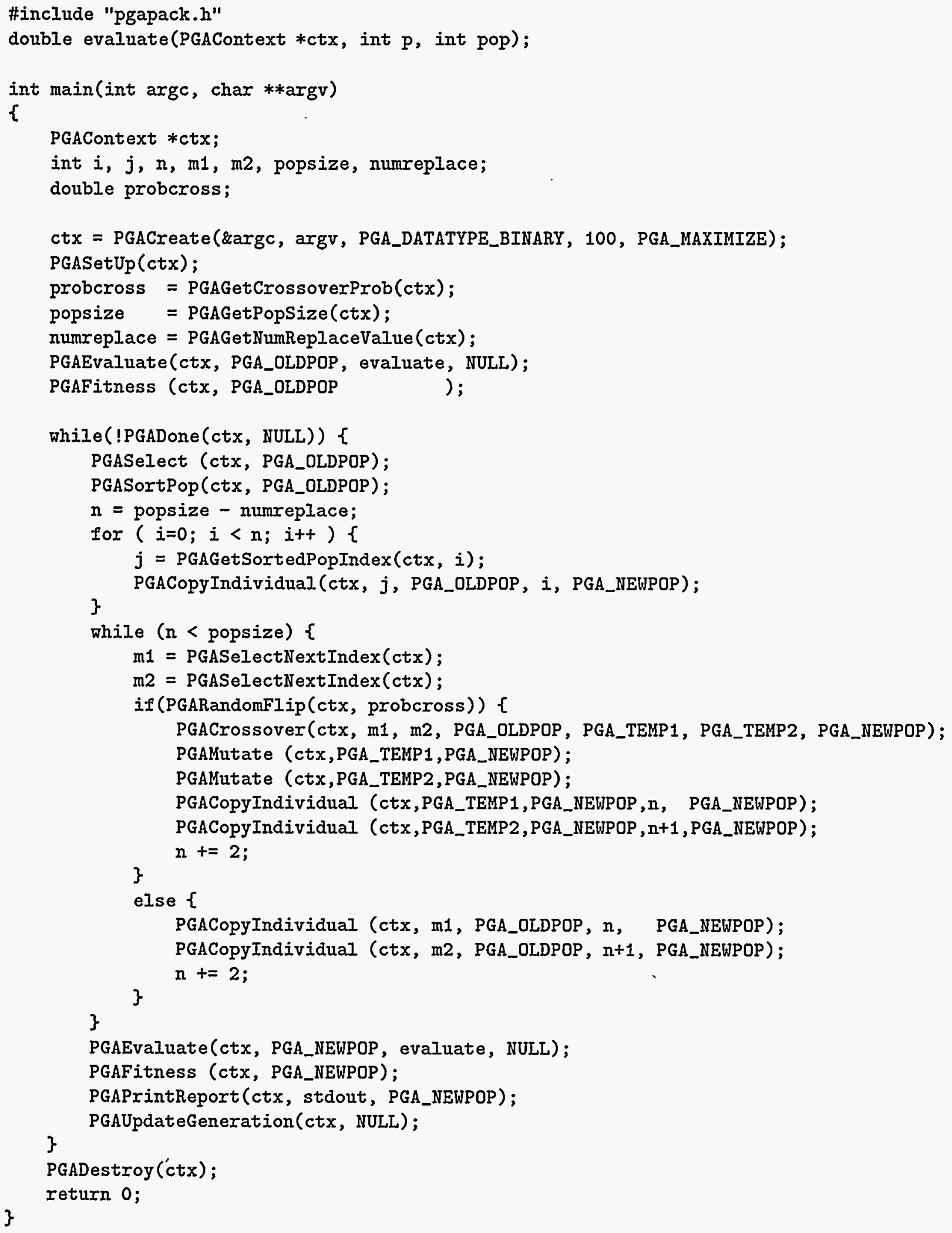

Figure 6.2: Example of Explicit Usage 


\subsection{Explicit PGAPack Functions}

This section briefly discusses other functions not shown in the previous examples or discussed in Chapter 5 . Additional information about these and other PGAPack functions is contained in Appendix B (function bindings) and the ./examples directory.

PGARunMutationAndCrossover and PGARunMutationOrCrossover perform the recombination step. The former applies mutation to strings that undergo crossover. The latter applies only mutation to strings that did not undergo crossover.

The restart operator described earlier in Section 5.8 can be invoked explicitly with PGARestart(ctx, oldpop, newpop), where the best string from population oldpop is used to initialize population newpop.

PGADup I icate (ctx, $p, P G A$ OLDPOP, PGA_NEWPOP , 20) returns PGA_TRUE if string $p$ in population PGA_OLDPOP is a duplicate of any of the first 20 strings in population PGA_NEWPOP. PGAChange(ctx, p, PGA_OLDPOP) repeatedly applies the mutation operator to string $\mathrm{P}$ in population PGA_OLDPOP until at least one mutation has occurred.

In PGAPack three values are associated with each string: (1) the user's evaluation function value, (2) a Boolean flag to indicate whether the evaluation function value is up to date with respect to the actual string, and (3) the fitness value. If PGARun is not used, the user must manage these values explicitly.

PGAEvaluate (ctx, PGA_NEWPOP, evaluate, comm) will execute the user's evaluation function, evaluate, on each string in population PGA_NEWPOP that has changed (for example, from crossover) since its last evaluation. PGAEvaluate will set both the evaluation function value and associated Boolean flag automatically. The argument comm is an MPI communicator. Valid values are NULL for an explicitly sequential example, or any valid MPI communicator. Depending on the number of processes specified when the program was invoked, and the value of the comm argument, PGAEvaluate may be run with one or more processes. See Chapter 10 for further discussion.

PGAFitness will calculate the population fitness values from the evaluation function values. It is an error to call PGAFitness if all the evaluation function values are not up to date.

These same three values may be read also. PGAGetEvaluation(ctx, p, PGA OLDPOP) returns the evaluation function value. PGAGetEvaluationUpToDateFlag(ctx, p, PGA_OLDPOP) returns PGA_TRUE or PGA_FALSE to indicate whether the evaluation is up to date with the actual string or not, respectively. If PGAPack was compiled for debugging,PGAGetEvaluation will print a warning message if the evaluation is not up to date. PGAGetFitness (ctx, p, PGA_OLDPOP) returns the fitness value.

At times, (e.g., applying a hill-climbing function) the user may need to explicitly set the evaluation function value and associated Boolean flag (fitness values can be calculated only by calling PGAFitness). PGASetEvaluation(ctx, p, PGA_OLDPOP, 123.4) will set the evaluation function value to 123.4 and the associated Boolean flag to PGA_TRUE. The Boolean flag may be set independently with PGASetEvaluationUpToDateFlag. For example, PGASetEvaluationUpToDateFlag (ctx, p, PGA_OLDPOP, PGA_FALSE) sets the status of the Boolean flag of string $p$ in population PGA_OLDPOP to out of date.

PGAMean ( $\operatorname{ctx}, a, n$ ) returns the mean of the $n$ values in array a. PGAStddev (ctx, a, $n$, mean) returns the standard deviation of the $n$ values in array a whose mean is mean. PGARank (ctx, $p$, order, $n$ ) returns an index that is the rank of string $p$ as given by the sorted array order of length $n$.

PGAGetPrintFrequency(ctx) returns the frequency with which GA statistics are reported. PGAGetWorstIndex (ctx, PGA_OLDPOP) returns the index of the string in population PGA_OLDPOP with the worst evaluation function value. PGAGetBestIndex (ctx, PGA_OLDPOP) returns the index of the string in population PGA_OLDPOP with the best evaluation function value. 


\section{Chapter 7}

\section{Custom Usage: Native Data Types}

This chapter discusses how PGAPack may be extended by replacing some of the standard PGAPack functions with user-defined functions for use with one of PGAPack's four native data types. This can be done from both $\mathrm{C}$ and Fortran.

\subsection{Basics}

In PGAPack, high-level (data-structure-neutral) functions call data-structure-specific functions that correspond to the data type used. The implementation uses function pointers that, by default, are set to the correct values for the datatype used. The user may change these defaults and set the function pointers to execute their functions instead. The functions the user can substitute for are initialization, crossover, mutation, checking for duplicate strings, string printing, termination criteria, and a generic function called at the end of each GA iteration.

The function call PGASetUserFunction (ctx, PGA_USERFUNCTION_MUTATION, mymute) will cause PGAPack to execute the function mymute whenever the mutation operator is called. Table 7.1 is a list of functions that can be customized for use with a native datatype. The first column describes the functionality, and the second column the symbolic constant for use with PGASetUserFunction. The calling sequence for these functions is fixed and must follow the function prototypes in Table 7.2. The files ./examples/templates/uf native.c and ./examples/templates/uf_native.f contain template routines for these functions. A specific example is given below.

Checking the termination criteria requires some discussion. The function PGADone will either check to see if the standard stopping criteria (see Section 5.3) have been met, or call the user function specified by PGA_USERFUNCTION_STOPCOND. If you wish to have the user function check for the standard stopping criteria in addition to whatever else it does, it should call PGACheckStoppingConditions (ctx). Do not call PGADone as this will cause an infinite loop to occur. Note that in a parallel program PGACheckStoppingConditions should only be called by the master process (see Chapter 10).

The end of generation function (which is null by default) may be used for gathering statistics about the GA, displaying custom output, etc. This function is called after all generational computation is complete, but

Table 7.1: Customizeable Functions: Native Data Types

\begin{tabular}{|l|l|}
\hline \hline \multicolumn{1}{|c|}{ Functionality } & \multicolumn{1}{|c|}{ Symbolic Constant } \\
\hline Initialization & PGA_USERFUNCTION_INITSTRING \\
Crossover & PGA_USERFUNCTION_CROSSOVER \\
Mutation & PGA_USERFUNCTION_MUTATION \\
Duplicate Checking & PGA_USERFUNCTION_DUPLICATE \\
String Printing & PGA_USERFUNCTION_PRINTSTRING \\
Termination Criteria & PGA_USERFUNCTION_STOPCOND \\
End of generation & PGA_USERFUNCTION_ENDOFGEN \\
\hline
\end{tabular}


Table 7.2: Calling Sequences for Customizable Functions

\begin{tabular}{|l|r|l|}
\hline \hline \multicolumn{1}{|c|}{ Symbolic Constant } & Return & \multicolumn{1}{|c|}{ Function Prototype } \\
\hline PGA_USERFUNCTION_INITSTRING & void & (PGAContext*, int, int) \\
PGA_USERFUNCTION_CROSSOVER & void & (PGAContext*, int, int, int, int, int, int) \\
PGA_USERFUNCTION_MUTATION & int & (PGAContext*, int, int, double) \\
PGA_USERFUNCTION_DUPLICATE & int & (PGAContext*, int, int, int, int) \\
PGA_USERFUNCTION_PRINTSTRING & void & (PGAContext*, FILE $*$, int, int) \\
PGA_USERFUNCTION_STOPCOND & int & (PGAContext*) \\
PGA_USERFUNCTION_ENDOFGEN & void & (PGAContext*) \\
\hline
\end{tabular}

before the population pointers (PGA_NEWPOP, PGA_OLDPOP) have been switched and the standard PGAPack output printed. Therefore, be sure to use PGA $\_$NEWPOP as the population pointer. There is no mechanism for suppressing the standard PGAPack generational output.

\subsection{Example Problem: C}

The example problem in Figure 7.1 is to maximize $\sum_{j=1}^{L} x_{j}$ with $1 \leq x_{j} \leq L$, where $L$ is the string length. The optimal solution to this problem, $L^{2}$, is achieved by setting each $x_{j}$ to $L$. The files for this example, ./examples/maxint.c and ./examples/maxint.f, contain template routines for these functions.

The example shows the use of a custom mutation function with an integer data type. The PGASetUserFunction function specifies that this function, MyMutation, will be called when the mutation operator is applied, rather than the default mutation operator. MyMutation generates a random integer on the interval $[1, L]$.

\subsection{Example Problem: Fortran}

Figure 7.2 is the same example as in Figure 7.1 written in Fortran. 


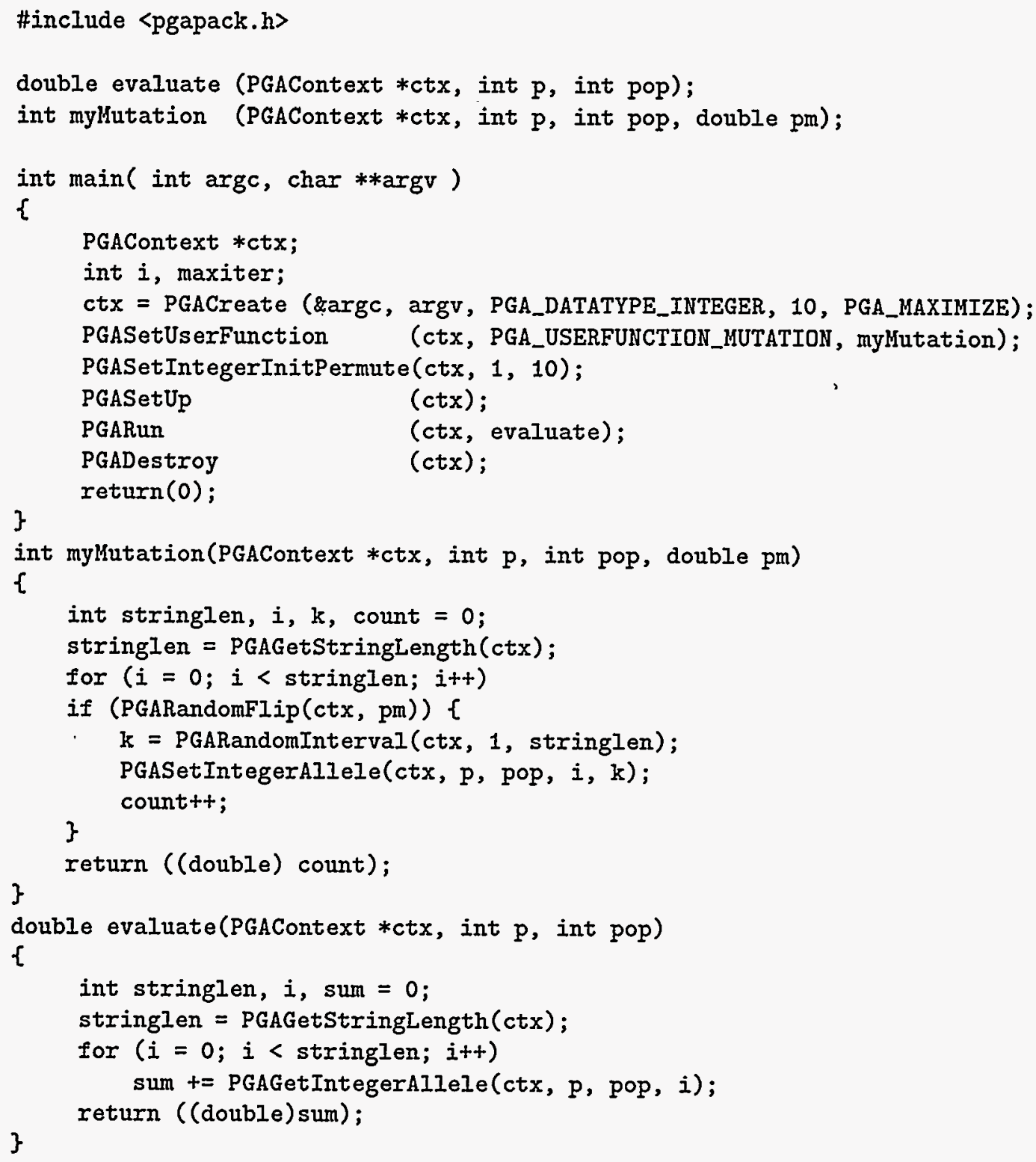

Figure 7.1: PGAPack C Example Using Custom Mutation Operator 


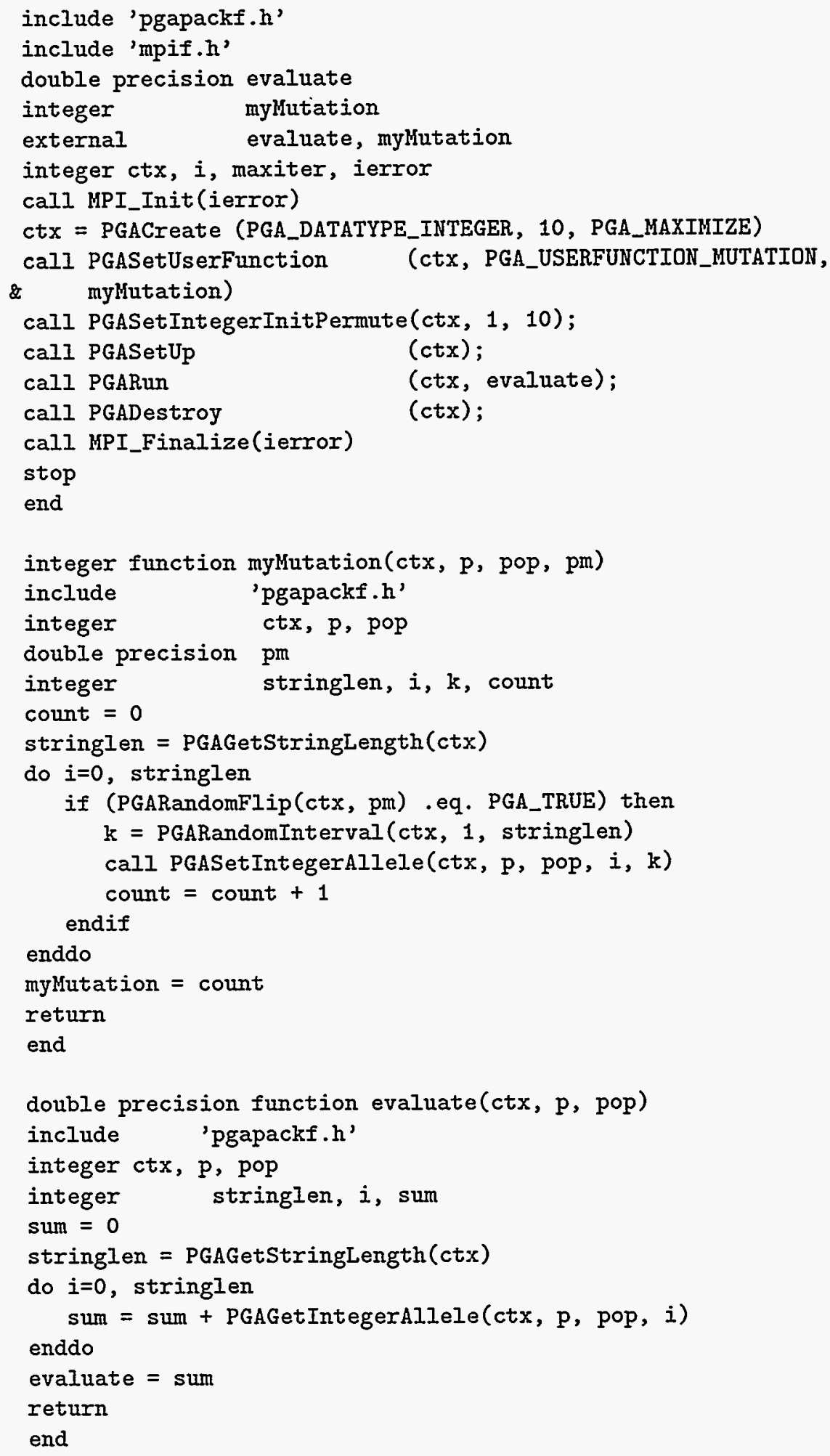

Figure 7.2: PGAPack Fortran Example Using Custom Mutation Operator 


\section{Chapter 8}

\section{Custom Usage: New Data Types}

This chapter discusses how PGAPack may be extended by defining a new data type. Defining a new data type may be done only in $\mathrm{C}$ programs.

\subsection{Basics}

To create a new data type, you must (1) specify PGA_DATATYPE_USER for the datatype in the PGACreate call and (2) for each entry in Table 8.1, call PGASetUserFunction to specify the function that will perform the given operation on the new data type. If the data type is PGA_DATATYPE_USER, the string length specified to PGACreate can be whatever the user desires. It will be returned by PGAGetStringLength but is not otherwise used in the data-structure-neutral functions of PGAPack.

The calling sequences for the functions in Table 8.1 are given in Table 8.2. The file ./examples/templates/uf_new.c contains template routines for these functions.

While PGAPack requires that the user supply all the functions in Table 8.1, your program may not require the functionality of all of them. For example, the user really does not need to write a function to pack the strings for message-passing unless a parallel version of PGAPack is being used. In these cases, we suggest that the user supply a stub function; i.e., a function with the correct calling sequence but no functionality.

\subsection{Example Problem}

This example illustrates use of a user-defined structure as the new data type. The problem is one of molecular docking where one protein molecule (the ligand) is to be docked into a second, target protein molecule. Figure 8.1 contains the function prototypes for each function that will operate on the new datatype, the definition of the user's structure (ligand), and the main program.

The first three doubles of the array $t$ in structure ligand represent the translation of the ligand molecule in the $x-, y$-, and $z$-axes, respectively. The last three doubles in the array $t$ represent the rotation of the

Table 8.1: Functions Required for New Data Types

\begin{tabular}{|l|l|}
\hline \hline \multicolumn{1}{|c|}{ Functionality } & \multicolumn{1}{|c|}{ Symbolic Constant } \\
\hline Memory allocation & PGA_USERFUNCTION_CREATESTRING \\
String packing & PGA_USERFUNCTION_BUILDDATATYPE \\
Mutation & PGA_USERFUNCTION_MUTATION \\
Crossover & PGA_USERFUNCTION_CROSSOVER \\
String printing & PGA_USERFUNCTION_PRINTSTRING \\
String copying & PGA_USERFUNCTION_COPYSTRING \\
Duplicate checking & PGA_USERFUNCTION_DUPLICATE \\
\hline
\end{tabular}


Table 8.2: Calling Sequences for New Data Type Functions

\begin{tabular}{|l|r|r|}
\hline \hline \multicolumn{1}{|c|}{ Symbolic Constant } & Return & Function Prototype \\
\hline PGA_USERFUNCTION_CREATESTRING & void & (PGAContext*, int, int, int) \\
PGA_USERFUNCTION_BUILDDATATYPE & int & (PGAContext*, int, int) \\
PGA_USERFUNCTION_MUTATION & int & (PGAContext*, int, int, double) \\
PGA_USERFUNCTION_CROSSOVER & void & (PGAContext*, int, int, int, int, int, int) \\
PGA_USERFUNCTION_PRINTSTRING & void & (PGAContext*, FILE *, int, int) \\
PGA_USERFUNCTION_COPYSTRING & int & (PGAContext*, int, int, int, int) \\
PGA_USERFUNCTION_DUPLICATE & int & (PGAContext*, int, int, int, int) \\
\hline
\end{tabular}

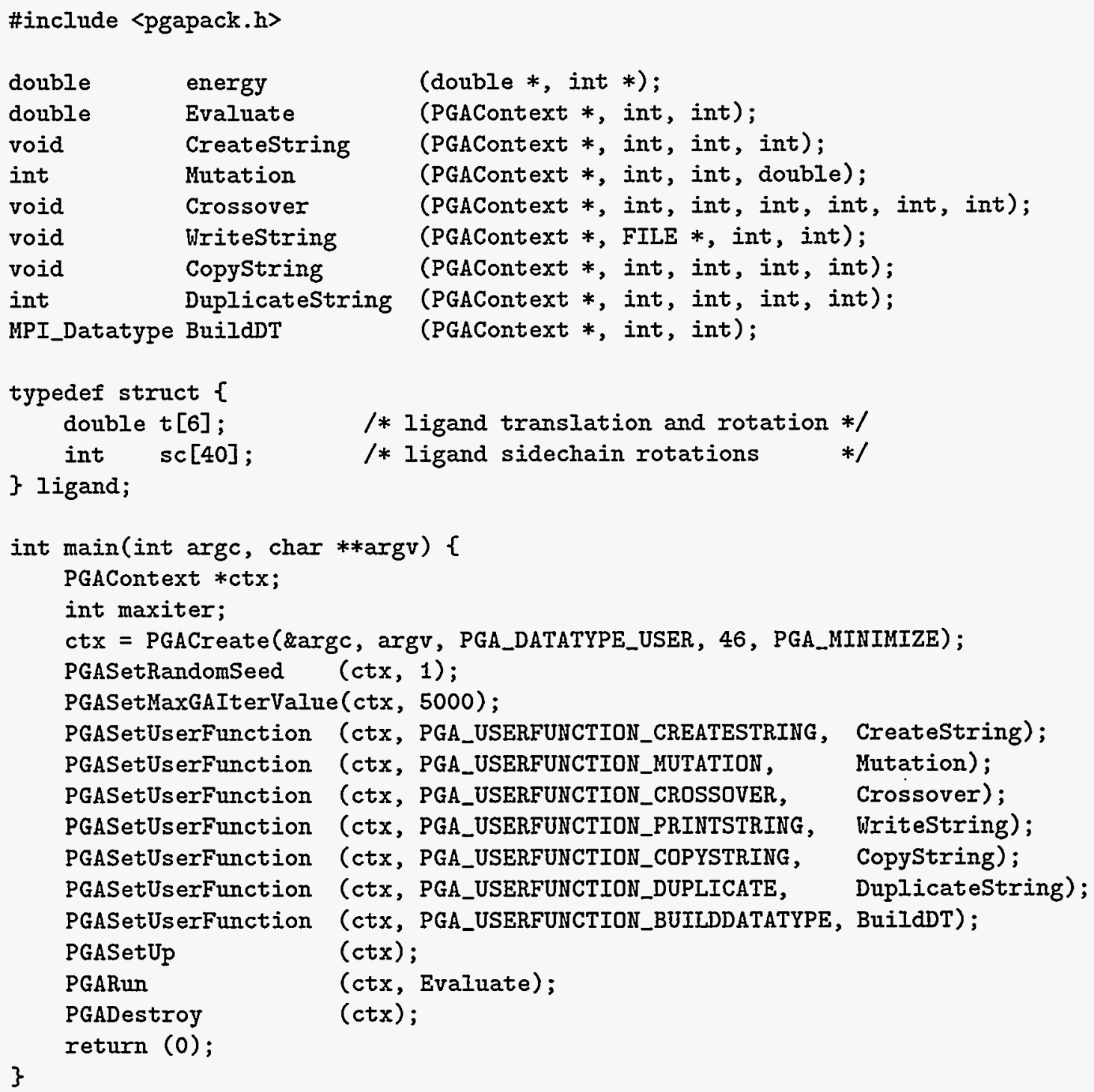

Figure 8.1: Main Program for Structure Data Type 


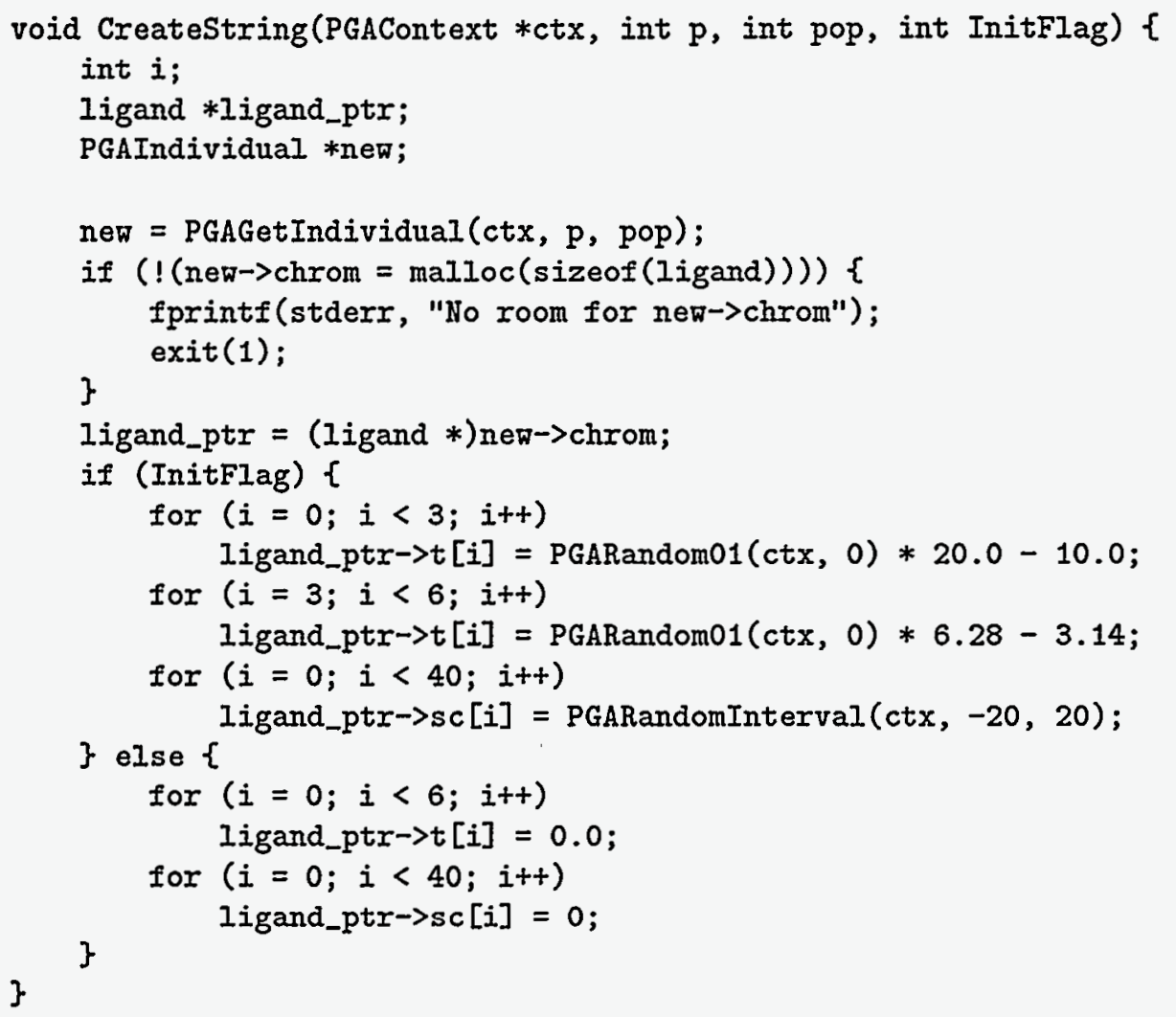

Figure 8.2: Creation and Initialization Function for Structure Data Type

ligand molecule about each of the axes. The ints in the sc array represent side chain rotations (which are discrete) of the ligand molecule.

Figure 8.2 contains the function CreateString that allocates and initializes the ligand structure. At this level of usage it is no longer always possible to maintain the ( $p, p o p)$ abstraction to specify an individual (a string and associated fields). CreateString works directly with the string pointer that ( $p, p \circ p)$ is mapped to. If InitFlag is true, CreateString will initialize the fields; otherwise they are set to 0 .

$P G A G e t$ Individual (ctx, $p, p o p$ ) returns a pointer of type PGAIndividual to the individual (the string and associated fields) specified by ( $p, p \circ p)$. PGAIndividual is a structure, one of the fields of which is chrom, a void pointer to the string itself. That pointer, new->chrom, is assigned the address of the memory allocated by the malloc function. As malloc returns a void pointer, no cast is necessary.

The value of InitFlag is passed by PGAPack to the user's string creation routine. It specifies whether to randomly initialize the string or set it to zero. By default, PGA_OLDPOP (except for PGA_TEMP1 and PGA_TEMP1 which are set to zero) is randomly initialized, and PGA_NEWPOP is set to zero. This choice may be changed with the PGASetRandomInitFlag function discussed in Section 5.4.)

Figure 8.3 contains the mutation function Mutation for the ligand data type. Each of the 46 genes has a probability of $m r$ of being changed. If a mutation occurs, Mutation adds or subtracts one tenth to the existing value of a double, and adds or subtracts one to an int.

Figure 8.4 contains the crossover function Crossover, which implements uniform crossover for the ligand data type. The lines

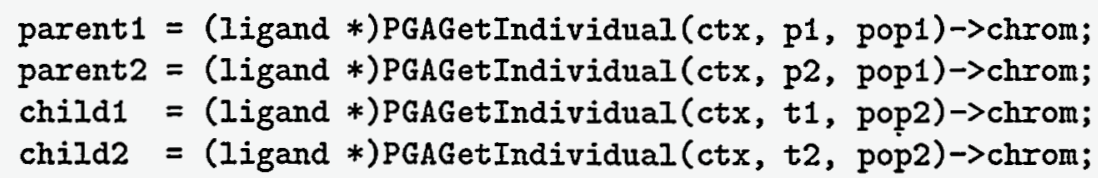




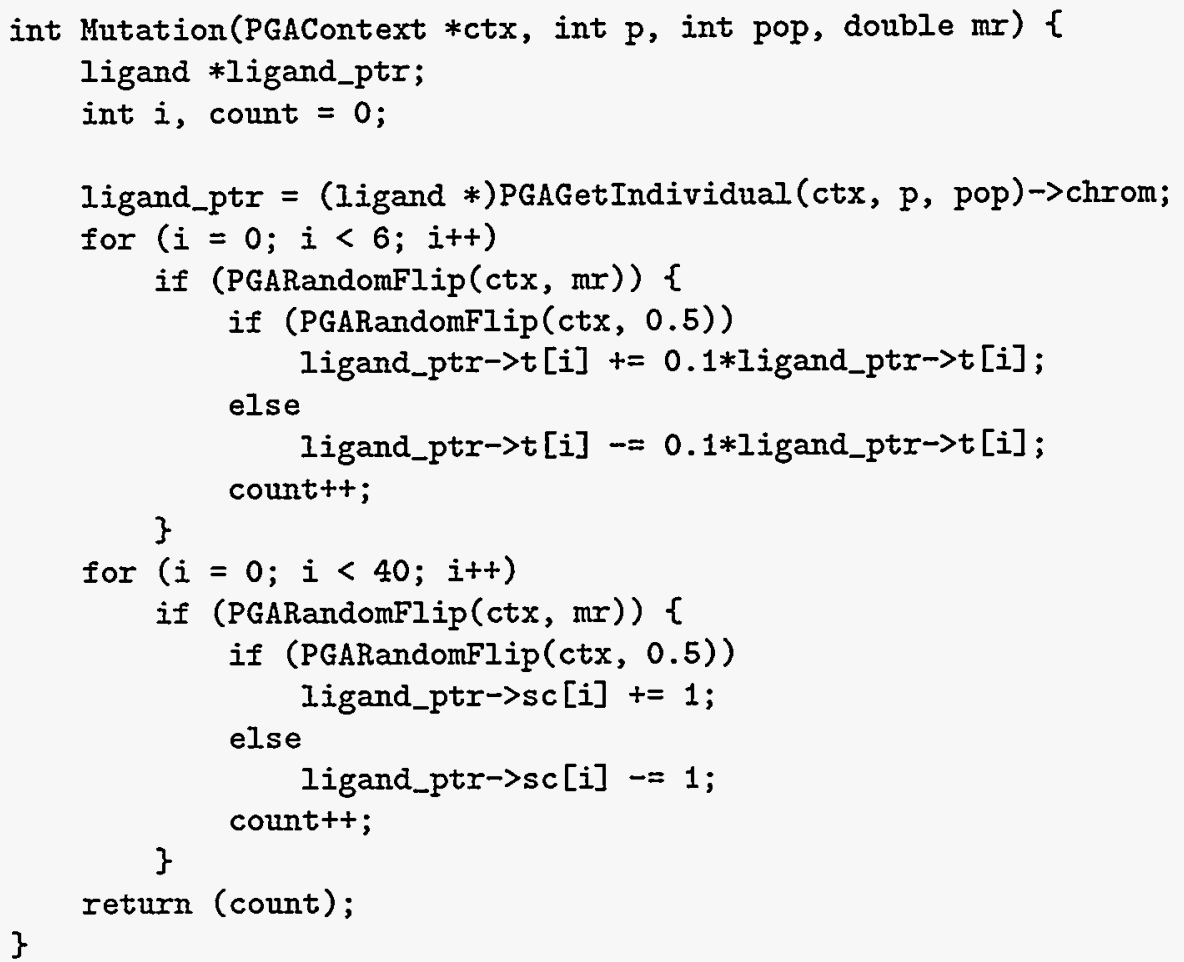

Figure 8.3: Mutation for Structure Data Type

are worthy of mention. Each implements in one line what the two lines

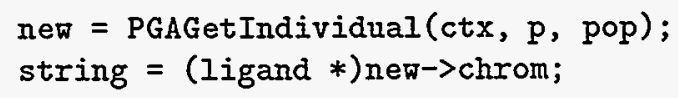

in Mutation did. Either style is acceptable. PGAGetIndividual returns a pointer whose chrom field (a void pointer) is cast to the ligand data type.

Figure 8.5 contains the code for DuplicateString, which checks for duplicate ligand structures. It uses the ANSI $\mathrm{C}$ memcmp function for this purpose.

Figure 8.6 contains the evaluation function for this example. It again uses PGAGetIndividual to map ( $p, p o p$ ) into a pointer to the string of interest. For user data types, PGAPack does not provide a PGAGetUserAllele function, so access to the allele values is made directly through the pointer.

Figure 8.7 contains the function BuildDT that builds an MPI datatype for sending strings to other. processors. Consult an MPI manual for further information. 
void Crossover(PGAContext $* \mathrm{ctx}$, int $\mathrm{p} 1$, int $\mathrm{p} 2$, int pop1, int $t 1$, int $t 2$,

int i;

int pop2) \{

ligand *parent1, *parent2, *child1, *child2;

double pu;

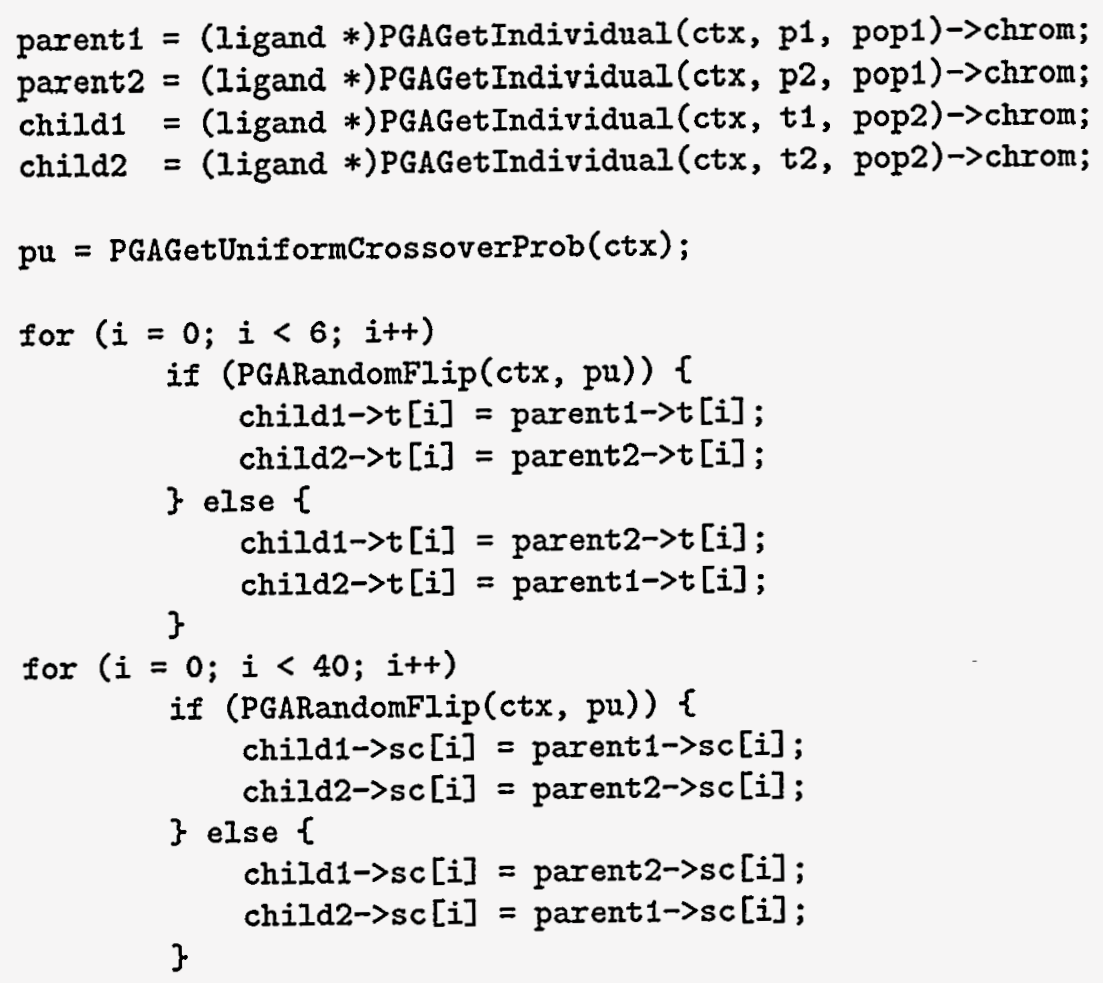

Figure 8.4: Crossover for Structure Data Type

int DuplicateString(PGAContext $* \operatorname{ctx}$, int $\mathrm{p} 1$, int pop1, int $\mathrm{p} 2$, int pop2) \{ void $* a, * b$;

$\mathrm{a}=$ PGAGetIndividual (ctx, $\mathrm{p} 1$, pop1) $\rightarrow$ chrom;

$\mathrm{b}=$ PGAGetIndividual (ctx, p2, pop2)->chrom;

3

return (!memcmp $(a, b, \operatorname{sizeof}($ ligand)) );

Figure 8.5: Duplicate Testing for Structure Data Type 


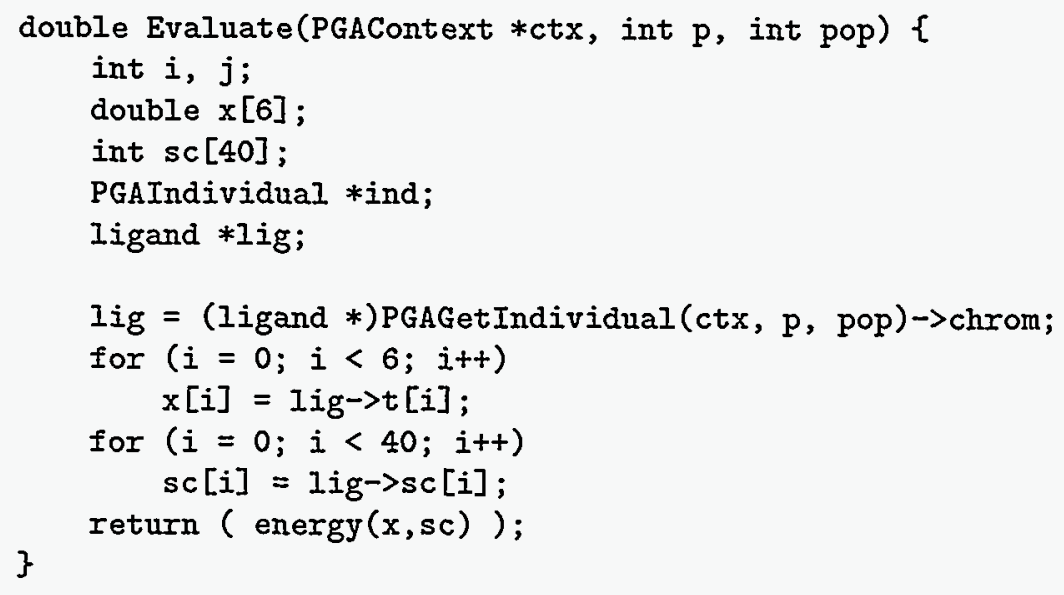

Figure 8.6: Evaluation Function for Structure Data Type 


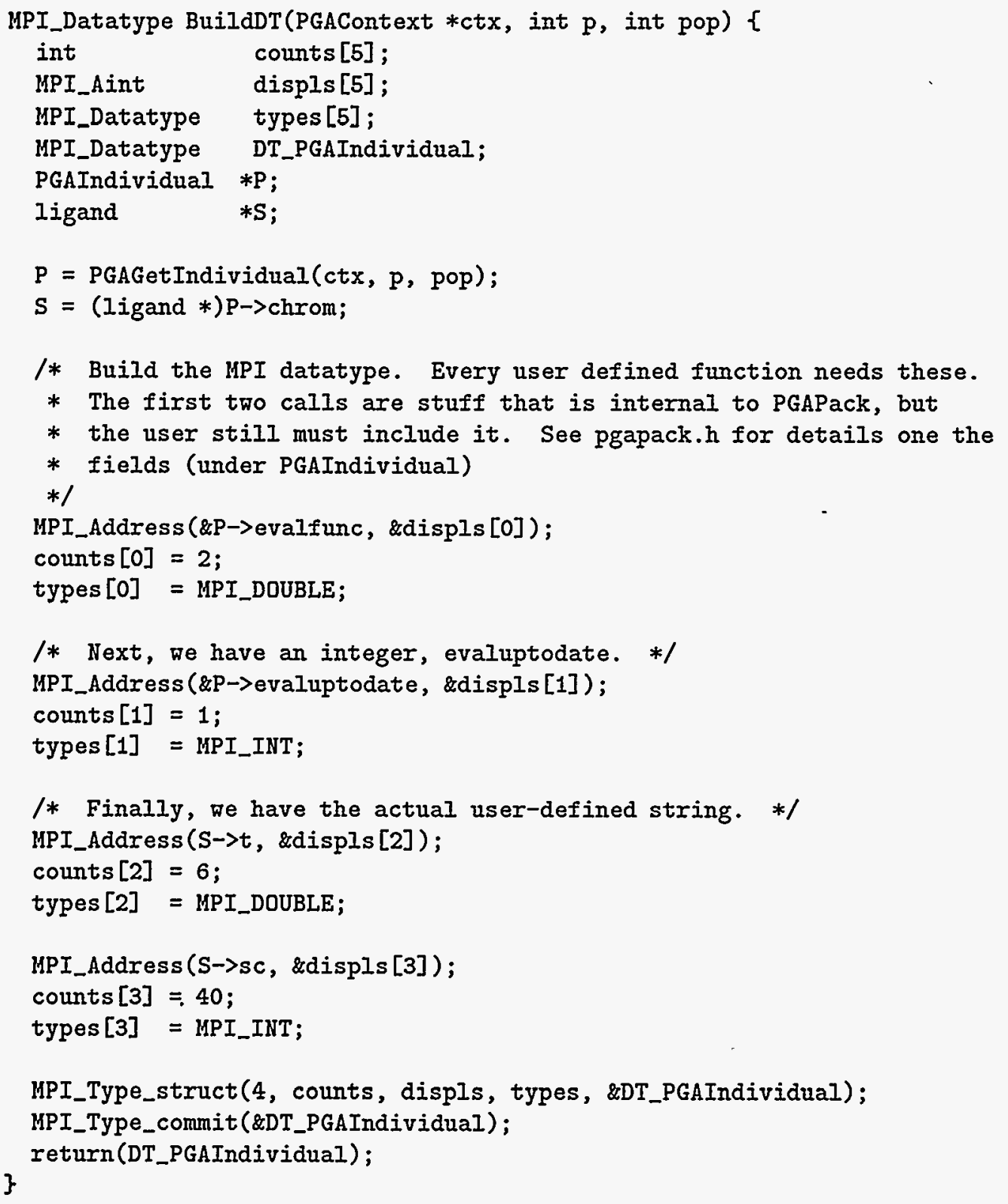

Figure 8.7: Message Packing Function for Structure Data Type 


\section{Chapter 9}

\section{Hill-Climbing and Hybridization}

Hill-climbing heuristics attempt to improve a solution by moving to a better neighbor solution. Whenever the neighboring solution is better than the current solution, it replaces the current solution. Genetic algorithms and hill-climbing heuristics have complementary strong and weak points. GAs are good at finding promising areas of the search space, but not as good at fine-tuning within those areas. Hill-climbing heuristics, on the other hand, are good at fine-tuning, but lack a global perspective. Practice has shown that a hybrid algorithm that combines GAs with hill-climbing heuristics often results in an algorithm that can outperform either one individually.

There are two general schemes for creating hybrid algorithms. The simplest is to run the genetic algorithm until it terminates and then apply a hill-climbing heuristic to each (or just the best) string. The second approach is to integrate a hill-climbing heuristic with the genetic algorithm. Choices to be made in the second case include how often to apply the hill-climbing heuristic and how many strings in the population to apply it to.

PGAPack supports hybrid schemes in the following ways:

- By passing, the context variable as a parameter to the user's hill-climbing function, the user has access to solution and parameter values, debug flags, and other information.

- The functions PGAGetBinaryallele, PGAGetIntegerallele, PGAGetRealallele, and PGAGetCharacterAllele allow the user's hill-climbing function to read allele values, and the functions PGASetBinaryAllele, PGASetIntegerAllele, PGASetRealAllele, and PGASetCharacterAllele allow the user's hill-climbing function to set allele values explicitly.

- The functions PGADecodeRealAsBinary, PGADecodeRealAsGrayCode, PGADecodeIntegerAsBinary, and PGADecodeIntegerAsGrayCode allow the user's hill-climbing function to read integer or real numbers encoded as binary or Gray code strings.

- The functions PGAEncodeRealAsBinary, PGAEncodeRealAsGrayCode, PGAEncodeIntegerAsBinary, and PGAEncodeIntegerAsGrayCode allow the user's hill-climbing function to encode integer or real numbers as binary or Gray code strings.

- The functions PGAGetEvaluation and PGASetEvaluation allow the user's hill-climbing function to get and set evaluation function values, and PGASetEvaluationUpToDateFlag and PGAGetEvaluationUPToDateFlag to get and set the flag that indicates whether an evaluation function value is up to date.

One way to run a hybrid GA and use PGARun is to use the PGASetUserFunction discussed in Chapter 7 to specify a user function to be called at the end of each GA iteration. A more flexible approach would be for the user to call the high-level PGAPack functions, and their hillclimber to explicitly specify the steps of the hybrid GA.

Figure 9.1 is a version of the Maxbit problem given in Section 3.1. It uses the hill-climbing function hillclimb, which is called after the recombination step. It randomly selects a gene to set to one. Note the PGASetEvaluationUpToDateFlag call. It sets the flag that indicates the evaluation function is not current 
with the string (since the string was changed). It is critical that this flag be set when the user changes a string, since the value of this flag determines whether PGAEvaluate will invoke the user's function evaluation routine.

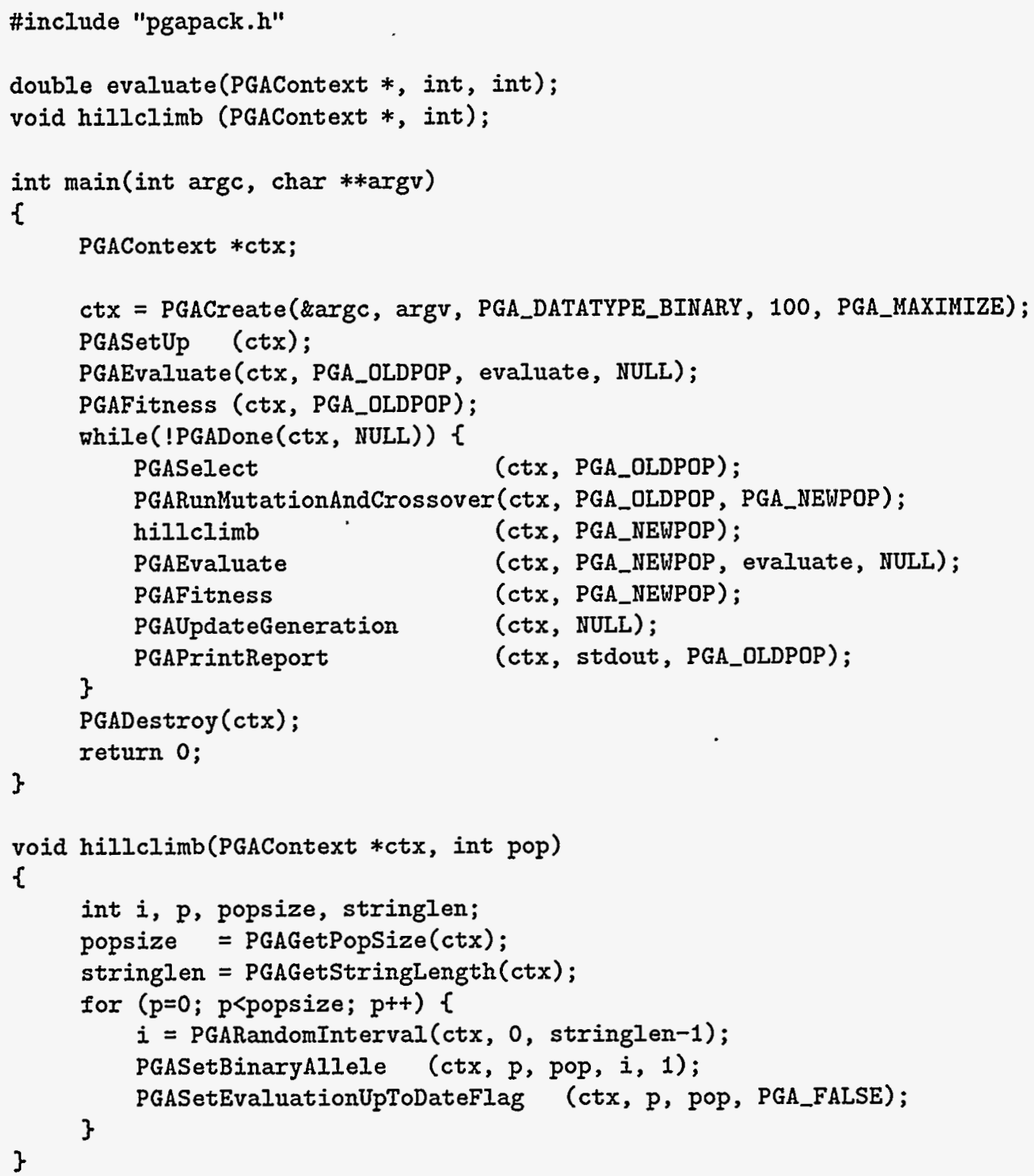

Figure 9.1: Hill-Climbing Heuristic for Maxbit Example 


\section{Chapter 10}

\section{Parallel Aspects}

This chapter assumes familiarity with the background material in Appendix C. It also assumes that a parallel version of PGAPack was built and that programs are linked with an MPI library (see Section 2.4).

Version 1.0 of PGAPack supports parallel and sequential implementations of the single population global model (GM). The parallel implementation uses a master/slave algorithm in which one process, the master, executes all steps of the genetic algorithm except the function evaluations. The function evaluations are executed by the slave processes ${ }^{1}$.

\subsection{Basic Usage}

Both sequential and parallel versions of PGAPack may be run by using PGARun. The choice of sequential or parallel execution depends on the number of processes specified when the program is started. If one process is specified, the sequential implementation of the GM is used (even in a parallel version of PGAPack). If two or more processes are specified, the parallel implementation of the GM is used. The examples in Chapter 3 can all be run in parallel by specifying more than one process at startup.

The specification of the number of processors is done at run time. The actual format of the specification depends on the MPI implementation and computer used (see Appendix C for some examples). PGARun uses the default MPI communicator, MPI_COMM_WORLD. This specifies that all processes specified at startup participate in the computation: one as the master process, the others as slave processes. A different communicator may be specified with PGASetCommunicator(ctx, comm), where comm is an MPI communicator.

PGARun is really a "wrapper" function that calls PGARunGM with the MPI_COMM_WORLD communicator. The user may call PGARunGM directly, that is, PGARunGM (ctx, evaluate, MPI_COMM_WORLD) where evaluate is the name of the user's evaluation function and the third argument is an MPI communicator. Note that the communicator specified by PGASetCommunicator does not affect PGARunGM.

\subsection{Explicit Use}

In general, explicit use of the parallel features is more complicated than in the case of sequential functions. This is because the user's program must coordinate the execution threads of multiple processes. PGARunGM encapsulates all that is necessary into one routine, and parts of its source code may serve as a useful starting point if one wishes to develop an explicitly parallel program. The parallel functions in PGAPack may be viewed as a hierarchy with PGARun and PGARunGM at the top of the hierarchy, PGAEvaluate next, PGASendindividual, PGAReceiveIndividual, and PGASendReceiveIndividual next, and PGABuildDatatype at the bottom of the hierarchy.

PGAGetRank (ctx, comm) returns the rank of the process in communicator comm. If comm is NULL it returns 0 . PGAGetNumProcs (ctx, comm) returns the number of processes in communicator comm. If comm is NULL it returns 1 .

\footnotetext{
${ }^{1}$ In the special case of exactly two processes, the master executes function evaluations as well.
} 
The type of algorithm used to execute PGAEvaluate(ctx,pop,f,comm) will depend on the number of processes in the communicator comm. If it is NULL or contains one process, a sequential implementation will be used. If more than one process is specified it will execute a master/slave evaluation of the strings in population pop that require evaluation by applying, $f$, the user's evaluation function. PGAEvaluate should be called by all processes in communicator comm.

PGASendIndividual (ct $x, p$, pop, dest, tag, comm) will send string $p$ in population pop to process dest. tag is a tag used to identify the message, and comm is an MPI communicator. This function calls MPI_Send to perform the actual message passing. In addition to string $\mathrm{p}$ itself, the evaluation function value, fitness function value, and evaluation status flag are also sent.

PGAReceiveIndividual is the complementary function to PGASendIndividual. For example, PGAReceiveIndividual (ctx, $p$, pop, source, tag, comm, status) will store in location $p$ in population pop the string and fields of the individual sent from process source with the MPI tag tag and MPI communicator comm. status is an MPI status vector.

PGASendReceiveIndividual combines the functionality of PGASendIndividual and PGAReceiveIndividual. This may be useful in avoiding potential deadlock on some systems. For example, PGASendReceiveIndividual (ctx, sp, spop, dest, stag, rp, rpop, source, rtag, comm, status). Here, sp is the index of the string in population spop to send to process dest with tag stag. The string received from process source with tag rtag is stored in location $x p$ in population rpop. comm and status are the same as defined earlier.

PGABuildDatatype(ctx,p,pop) packs together the string and fields that PGASendIndividual, PGAReceiveIndividual, and PGASendReceiveIndividual send and receive. The result is of type MPIDatatype.

\subsection{Example}

Figure 10.1 is a parallel version of the example in Figure 6.1. Since we now have multiple processes executing the program at the same time, we must coordinate each ones execution. In the example, the master process (the one with rank 0 as determined by PGAGetRank) executes all functions, and the slave processes execute only those functions that take a communicator as an argument. Note that this example will execute correctly even if only one process is in the communicator.

\subsection{Performance}

The parallel implementation of the GM will produce the same result as the sequential implementation, usually faster. However, the parallel implementation varies with the number of processes. If two processes are used, both the master process and the slave process will compute the function evaluations. If more than two processes are used, the master is responsible for bookkeeping only, and the slaves for executing the function evaluations. In general, the speedup obtained will vary with the amount of computation associated with a function evaluation and the computational overhead of distributing and collecting information to and from the slave processes.

The speedup that can be achieved with the master/slave model is limited by the number of function evaluations that can be executed in parallel. This number depends on the population size and the number of new strings created each generation. For example, if the population size is 100 and a 100 new strings are created each GA generation, then up to 100 processors can be put to effective use to run the slave processes. However, with the default rule of replacing only $10 \%$ of the population each GA generation, only 10 processors can be used effectively. 


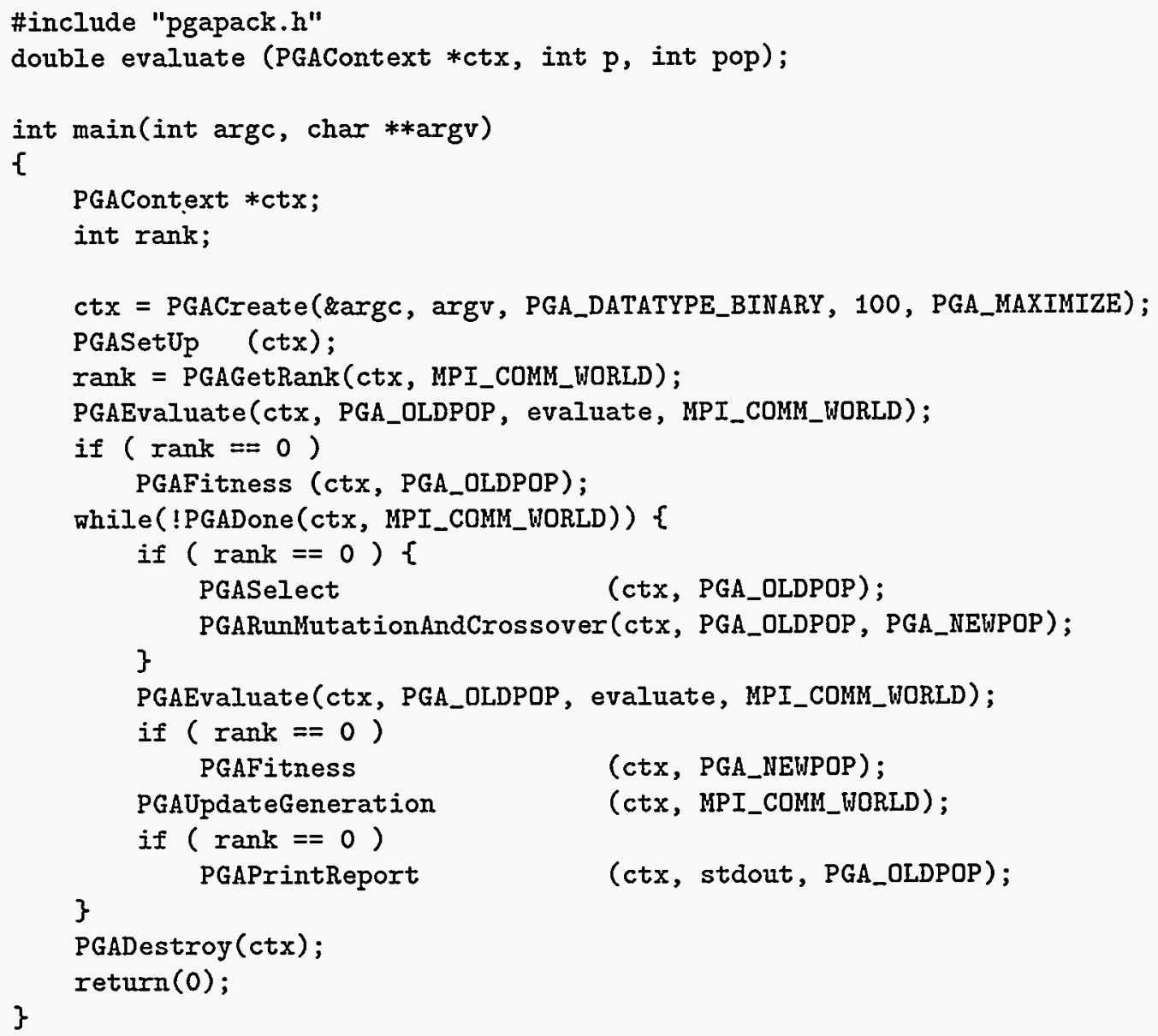

Figure 10.1: Simple Parallel Example of Explicit Usage 


\section{Chapter 11}

\section{Fortran Interface}

PGAPack is written entirely in ANSI C. A set of interface functions, also written in C, is designed to be called by Fortran programs and then call the "real" $\mathrm{C}$ routine. This mechanism provides most of PGAPack's functionality to Fortran programs. The following list contains most major differences between $\mathrm{C}$ and Fortran. Additional, machine-specific idiosyncrasies are noted in Appendix D.

- The Makefiles for the Fortran examples (in ./examples/fortran and ./examples/mgh) are not configured to use the-I mechanism for specifying the include file search path (since not all Fortran compilers support this). Therefore, you will need to copy or set up a symbolic link to ./include/pgapackf.h from the directory you are compiling a Fortran program in.

- The context variable is declared integer (or integer*8, see Appendix D) in Fortran.

- PGACreate takes only three arguments in Fortran (not argc or argv as in C).

- The Fortran include file is pgapackf.h and should be included in any Fortran subroutine or function that calls a PGAPack function, to ensure correct typing and definition of functions and symbolic constants.

- If a $\mathrm{C}$ function returns an $\{$ int, double, pointer $\}$, the corresponding Fortran function returns an $\{$ integer, double precision, integer $\}$. If the $C$ function is void it is implemented as a Fortran subroutine.

- When supplying function arguments, a $C$ int corresponds to a Fortran integer, and a $\mathrm{C}$ double corresponds to a Fortran double precision. For example, to set the crossover probability to 0.6 , use call PGASetCrossoverProb(ctx, 0.6do), or double precision pc $\mathrm{pc}=0.6$ call PGASetCrossoverProb(ctx, pc)

- Gene indices are $[0, L-1]$ in $C$, and $[1, L]$ in Fortran, where $L$ is the string length.

- Population member indices are $[0, N-1]$ in $\mathrm{C}$, and $[1, N]$ in Fortran, where $N$ is the population size.

- Fortran does not support command line arguments (Section 5.13).

- Fortran allows custom usage with native data types (Chapter 7), but not with new data types (Chapter 8).

- In the MPICH implementation of MPI, the Fortran and $\mathrm{C}$ versions of MPI Init are different. If the main program is in $\mathrm{C}$, then the $\mathrm{C}$ version of MPI_Init must be called. If the main program is in Fortran, the Fortran version of MPI_Init must be called. Therefore, Fortran users of PGAPack with MPICH must call MPI_Init themselves since PGACreate, which calls MPI_Init if users haven't called it themselves, is written in $\mathrm{C}$. 
- The DEC Alpha and Silicon Graphics Power Challenge, which have 64-bit C pointers and 32-bit Fortran integers (but not the Cray T3D which has 64 -bit Fortran integers), have additional differences ${ }^{1}$. These arise because a Fortran integer is too small to hold the address returned by the $\mathrm{C}$ interface routine.

- The context variable should be declared integer*8.

- MPI_COMM_WORLD should not be passed directly to PGAPack Fortran functions. Instead, PGAGetCommunicator should be called to return the address into an integer*8 variable. For example

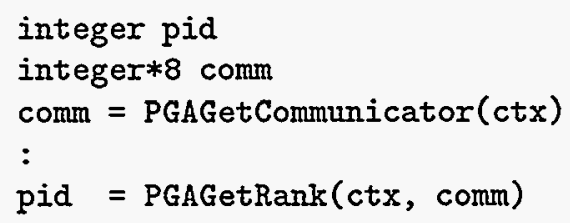

- MPI_COMM_WORLD can and should be passed directly to any MPI routines called directly from Fortran.

- Calling an MPI routine that returns a communicator is safe. However, passing the returned communicator to a PGAPack Fortran function will usually fail.

${ }^{1}$ More generally, these issues arise whenever the size of a Fortran integer is less than the size of a pointer. 


\section{Chapter 12}

\section{Debugging Tools}

PGAPack has a sophisticated built-in trace facility that is useful for debugging. When the facility is invoked, print statements to stdout allow the programmer to trace the sequence of functions PGAPack executes. Due to the negative impact on performance this facility is not available by default. Instead, you must explicitly enable tracing when configuring PGAPack with the -debug flag. See Section 2.4.

The trace facility uses the concept of a debug level. For example, executing the Maxbit example (Figure 3.1) with a debug level of 12, maxbit -pgadbg 12, will print the output shown in Figure 12.1. The "0:" is the process rank. This is followed by the name of a PGAPack function and the "action" that caused the print statement to execute. In this case, the action is entering the function. Note that the rank printed for a process is always its rank in the MPI_COMM_WORLD communicator, even if another communicator was set.

Tracing is enabled by specifying one or more debug levels to trace. A list of debug levels is given in Table 12.1. Not all debug level values are currently used. The values 1-10 are reserved for users as described below.

C programmers may set the debug level from the command line using either -pgadbg <debug level> or -pgadebug <debug level>. Several forms of the <debug level> argument are allowed. -pgadbg 12 will trace entering all high-level functions as shown in Figure 12.1. -pgadbg 12,13 or -pgadbg 12-13 will trace entering and exiting of all high-level functions. The command line option -pgahelp debug will list the debug level options and then exit.

Fortran (and C) programmers may access the trace facility via function calls. The function PGASetDebugLevel may be called to set a debug level. For example, call PGASetDebugLevel (ctx, 12) would produce the same output shown in Figure 12.1. PGAClearDebugLevel (ctx,12) will clear prints associated with debug level 12. PGAPrintDebugOptions(ctx) will print the list of available debug options.

The function PGASetDebugLevelByName will turn on debugging of the named function. For example, PGASetDebugLevelByName(ctx, "PGACrossover' ') will enable all the trace prints of PGACrossover. PGAClearDebugLevelByName will disable the tracing of the specified function.

Users can use the trace facility in their own functions (e.g., their evaluation function) in two ways. First, they can insert PGADebugPrint function calls in their functions using one of the symbolic constants defined in the header file pgapack.h. These are PGA_DEBUG_ENTERED, PGA_DEBUG_EXIT, PGA_DEBUG_MALLOC, PGA_DEBUG_PRINTVAR, PGA_DEBUG_SEND, and PGA_DEBUG_RECV for entering a function, exiting a function, allocating memory, print a variable's value, and sending or receiving a string, respectively.

For example, PGADebugPrint (ctx, PGA_DEBUG_ENTERED, "MyFunc", "Entered", PGA_VOID, NULL) will print the line
0 : MyFunc
: Entered

when the debug level of 12 is specified. PGADebugPrint(ctx, PGA_DEBUG_PRINTVAR, "MyFunc", " $i$ = ", PGA_INT, (void *) \&i) will print the line

$$
0: \text { MyFunc }: i=1
$$

when the debug level of 82 is specified. Users can also use the reserved debug levels of 1-10 to customize the trace facilities for use in their own functions. For example PGADebugPrint( $\operatorname{ctx}, 5$, "MyFunc", "After call to MyCleanUp", PGA_VOID, NULL); will print the line 


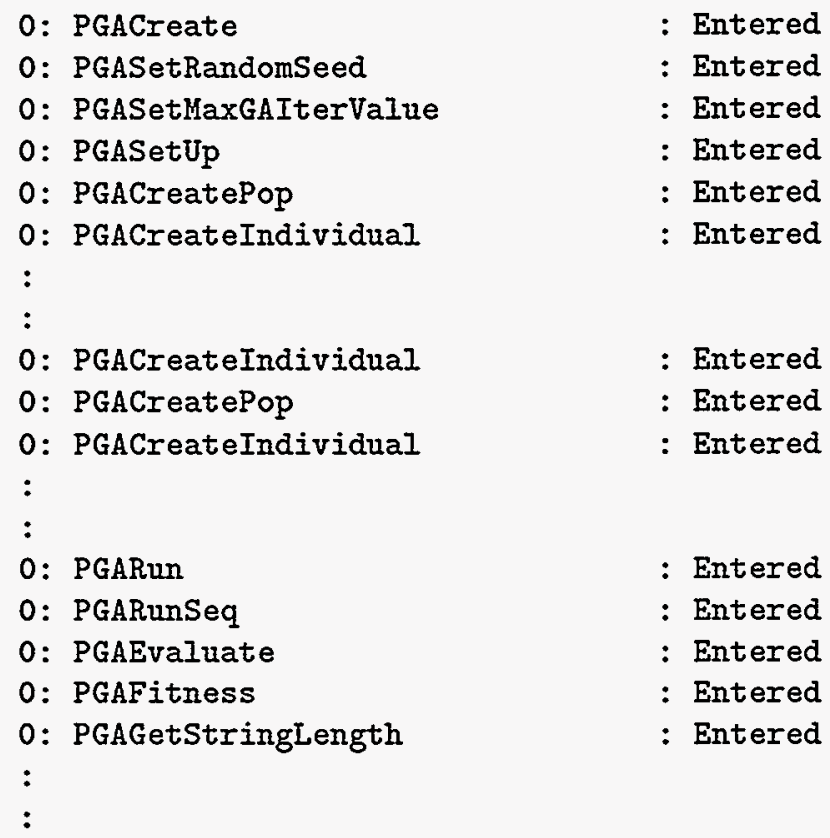

Figure 12.1: PGAPack Partial Trace Output for Maxbit Example
0 : MyFunc
: After call to MyCleanUp

when the debug level of five is specified.

Note that we use MPI_COMM_WORLD (1) for the random number seed and (2) for PGADebugPrint calls. 
Table 12.1: Debug Levels in PGAPack

\begin{tabular}{|r|l|}
\hline \hline 0 & Trace all debug prints \\
11 & Trace high-level functions \\
12 & Trace all function entries \\
13 & Trace all function exits \\
20 & Trace high-level parallel functions \\
21 & Trace all parallel functions \\
22 & Trace all send calls \\
23 & Trace all receive calls \\
30 & Trace Binary functions \\
32 & Trace Integer functions \\
34 & Trace Real functions \\
36 & Trace Character functions \\
40 & Trace population creation functions \\
42 & Trace select functions \\
44 & Trace mutation functions \\
46 & Trace crossover functions \\
48 & Trace function evaluation functions \\
50 & Trace fitness calculation functions \\
52 & Trace duplicate checking functions \\
54 & Trace restart functions \\
56 & Trace reporting functions \\
58 & Trace stopping functions \\
60 & Trace sorting functions \\
62 & Trace random number functions \\
64 & Trace system routines \\
66 & Trace utility functions \\
80 & Trace memory allocations \\
82 & Trace variable print statements \\
\hline
\end{tabular}




\section{Part III}

\section{Appendixes}


Appendix A

Default Values 
Table A.1: PGAPack Default Values

\begin{tabular}{|c|c|c|}
\hline CONCEPT & DEFAULT & SET WITH \\
\hline Population size & 100 & PGASetPopSize \\
\hline Copied for population replacement & PGA_POPREPL_BEST & PGASetPopReplacementType \\
\hline Stopping rule & PGA_STOP_MAXITER & PGASetStoppingRuleType \\
\hline Maximum iterations & 1000 & PGASetMaxGAIterValue \\
\hline Maximum no change iters & 100 & PGASetMaxiloChangeValue \\
\hline Max. population homogeneity before stopping & 95 & PGASetMaxSimilarityValue \\
\hline Number of new strings to generate & 10 & PGASetNumReplaceValue \\
\hline Apply mutation and crossover & PGA_FALSE & PGASetMutationAndCrossoverFlag \\
\hline Apply mutation or crossover & PGA_TRUE & PGASetMutationOrCrossoverFlag \\
\hline Crossover type & PGA_CROSSOVER_TWOPT & PGASetCrossoverType \\
\hline Probability of crossover & 0.85 & PGASetCrossoverProb \\
\hline Uniform crossover bias & $\overline{0.6}$ & PGASetUniformCrossoverProb \\
\hline Mutation type (Real strings) & PGA_MUTATION_GAUSSIAN & PGASetMutationType \\
\hline Mutation type (Integer strings) & PGA_MUTATION_PERMUTE & PGASetMutationType \\
\hline Mutation type (Character strings) & Same as initialization & PGASetCharacterInitType \\
\hline Mutation probability & $1 / \mathrm{L}$ & PGASetMutationProb \\
\hline Real mutation constant & 0.1 & PGASetMutationRealValue \\
\hline Integer mutation constant & 1 & PGASetMutationIntegerValue \\
\hline Mutation range bounded & PGA_TRUE & PGASetMutationBoundedFlag \\
\hline Select type & PGA_SELECT_TOURNAMENT & PGASetSelectType \\
\hline Probabilistic binary tournament par & 0.6 & PGASetPTournamentProb \\
\hline Use restart operator & PGA_FALSE & PGASetRestartFlag \\
\hline Restart frequency & 50 & PGASetRestartFrequencyValue \\
\hline Restart allele mutation rate & 0.5 & PGASetRestartAlleleChangeProb \\
\hline Allow duplicate strings & PGA_FALSE & PGASetNoDuplicatesFlag \\
\hline Fitness type & PGA_FITNESS_RAW & PGASetFitnessType \\
\hline Fitness type for minimization & PGA_FITNESSMIN_CMAX & PGASetFitnessMinType \\
\hline Multiplier for minimization problems & 1.01 & PGASetCMaxValue \\
\hline Parameter MAX in fitness by ranking & 1.2 & PGASetMaxFitnessRank \\
\hline Frequency of statistics printing & 10 & PGASetPrintFrequencyValue \\
\hline Print strings & PGA_FALSE & PGASetPrintoptions \\
\hline Print offline statistics & PGA_FALSE & PGASetPrintoptions \\
\hline Print online statistics & PGA_FALSE & PGASetPrintOptions \\
\hline Print best string & PGA_FALSE & PGASetPrintOptions \\
\hline Print worst string & PGAFALSE & PGASetPrint0ptions \\
\hline Print Hamming distance & PGA_FALSE & PGASetPrint0ptions \\
\hline Randomly initialize population & PGA_TRUE & PGASetRandomInitFlag \\
\hline Probability of initializing a bit to one & 0.5 & PGASetBinaryInitProb \\
\hline How to initialize real strings & Range & PGASetrealInitRange \\
\hline Real initialization range & {$[0,1]$} & PGASetReaIInitRange \\
\hline How to initialize integer strings & Permutation & PGASetIntegerInitPermute \\
\hline Integer initialization range & {$[0, L-1]$} & PGASetIntegerInitPermute \\
\hline How to initialize character strings & PGA_CINIT LOWER & PGASetCharacterInitFlag \\
\hline Seed random number with clock & PGA_TRUE & PGASetRandomSeed \\
\hline Default MPI communicator & MPI_COMM_WORLD & PGASetCommunicator \\
\hline
\end{tabular}

$L$ is the string length 


\section{Appendix B}

\section{Function Bindings}

\section{Symbolic Constants}

PGAPack defines many symbolic constants that are used as arguments to PGAPack functions. These constants are the same for both Fortran and C. Below is a list of these constants. These constants are the same for both Fortran and C.

- PGAPack Data Types

- PGA_DATATYPE_BINARY

- PGADATATYPE_INTEGER

- PGA_DATATYPE_REAL

- PGA_DATATYPE_CHARACTER

- PGA_DATATYPE_USER

- String Types

- PGABinary

- PGAInteger

- PGAReal

- PGACharacter

- Data Types used in PGAError Calls

- PGA_INT

- PGa_DOUBLE

- PGA_CHAR

- PGA_VOID

- True and False

- PGA_TRUE

- PGa_FALSE

- Miscellaneous PGAPack Flags

- PGA_FATAL

- PGA_WARNING

- PGA_UNINITIALIZED_INT 
- PGA_UNINITIALIZED_DOUBLE

- PGAPack Temporary and Population Constants

- PGA_TEMP1

- PGA_TEMP2

- PGA_OLDPOP

- PGA_NEWPOP

- Debug Levels

- PGA_DEBUG_ENTERED

- PGADEBUG_EXIT

- PGA_DEBUG MALLOC

- PGA_DEBUG_PRINTVAR

- PGADEBUG_SEND

- PGA_DEBUG_RECV

- Direction of Optimization

- PGA MAXIMIZE

- PGA_MINIMIZE

- Stopping Criteria

- PGA_STOP MAXITER

- PGA_STOP_NOCHANGE

- PGA_STOP_TOOSIMILAR

- Crossover

- PGA_CROSSOVER_DNEPT

- PGA_CROSSOVER_TWOPT

- PGA_CROSSOVER_UNIFORM

- Fitness

- PGAFITNESS_RAW

- PGA_FITNESS_NORMAL

- PGAFITNESS_RANKING

- Fitness Minimization

- PGAFITNESSMIN RECIPROCAL

- PGA_FITNESSMIN_CMAX

- Mutation Type

- PGA_MUTATION_CONSTANT

- PGA_MUTATION_RANGE

- PGA_MUTATION_UNIFORM

- PGA_MUTATION_GAUSSIAN

- PGA MUTATION PERMUTE 
- Population Replacement

- PGA_POPREPL_BEST

- PGA_POPREPL_RANDOM_NOREP

- PGA_POPREPL_RANDOM_REP

- Initialization Options

- PGA_CINIT IOWER

- PGA_CINIT_UPPER

- PGA_CINIT MIXED

- PGA IINIT PERMUTE

- PGA IINIT RANGE

- PGA_RINIT PERCENT

- PGA_RINIT RANGE

- Report Options

- PGA_REPORT_ONLINE

- PGA_REPORT_OFFLINE

- PGA_REPORT_HAMMING

- PGA_REPORT_STRING

- PGA_REPORT_WORST

- PGA_REPORT_AVERAGE

- Selection

- PGA_SELECT PROPORTIONAL

- PGA_SELECT_SUS

- PGA_SELECT_TOURNAMENT

- PGA_SELECT PTOURNAMENT

- User Functions

- PGA_USERFUNCTION_CREATESTRING

- PGA_USERFUNCTION_MUTATION

- PGA_USERFUNCTION_CROSSOVER

- PGA_USERFUNCTION_PRINTSTRING

- PGA_USERFUNCTION_COPYSTRING

- PGA_USERFUNCTION DUPLICATE

- PGA_USERFUNCTION_INITSTRING

- PGA_USERFUNCTION_BUILDDATATYPE

- PGA_USERFUNCTION_STOPCOND

- PGA_USERFUNCTION_ENDOFGEN 


\section{ANSI C Bindings}

The use of any PGAPack function requires that the user have \#include "pgapack. $h$ " at the top of the file that references PGAPack functions.

\begin{tabular}{|c|c|}
\hline Type & Function \\
\hline MPI_Datatype & PGABuildDatatype(PGAContext *ctx, int $\mathrm{p}$, int pop) \\
\hline void & PGAChange(PGAContext ${ }^{*} \operatorname{ctx}$, int $\mathrm{p}$, int pop) \\
\hline int & PGACheckStoppingConditions(PGAContext *ctx) \\
\hline int & PGACheckSum(PGAContext *ctx, int $p$, int pop) \\
\hline void & PGAClearDebugLevel(PGAContext *ctx, int level) \\
\hline void & PGAClearDebugLevelByName(PGAContext ${ }^{*}$ ctx, char ${ }^{*}$ funcname) \\
\hline void & PGACopyIndividual(PGAContext ${ }^{*} \mathrm{ctx}$, int p1, int pop1, int p2, int pop2) \\
\hline PGAContext* & PGACreate(int *argc, char ${ }^{* *}$ argv, int datatype, int len, int maxormin) \\
\hline void & PGACrossover(PGAContext ${ }^{*} \mathrm{ctx}$, int $\mathrm{p} 1$, int $\mathrm{p} 2$, int pop1, int $\mathrm{c} 1$, int $\mathrm{c} 2$, int pop2) \\
\hline void & $\begin{array}{l}\text { PGADebugPrint(PGAContext *ctx, int level, char *funcname, } \\
\text { char *msg, int datatype, void *data) }\end{array}$ \\
\hline void & PGADestroy(PGAContext *ctx) \\
\hline int & PGADone(PGAContext *ctx, MPI_Comm comm) \\
\hline int & PGADuplicate(PGAContext ${ }^{*} \mathrm{ct} x$, int $\mathrm{p}$, int pop1, int pop2, int $\left.\mathrm{n}\right)$ \\
\hline void & PGAEncodeIntegerAsBinary (PGAContext ${ }^{*} \mathrm{ctx}$, int $\mathrm{p}$, int pop, int start, int end, int val) \\
\hline void & PGAEncodeIntegerAsGrayCode(PGAContext ${ }^{*}$ ctx, int p, int pop, int start, int end, int val) \\
\hline void & $\begin{array}{l}\text { PGAEncodeRealAsBinary(PGAContext *ctx, int } p \text {, int pop, } \\
\text { int start, int end, double low, double high, double val) }\end{array}$ \\
\hline void & $\begin{array}{l}\text { PGAEncodeRealAsGrayCode(PGAContext *ctx, int p, int pop, } \\
\text { int start, int end, double low, double high, double val) }\end{array}$ \\
\hline void & PGAError(PGAContext ${ }^{*}$ ctx, char ${ }^{*} \mathrm{msg}$, int level, int datatype, void *data) \\
\hline void & $\begin{array}{l}\text { PGAEvaluate(PGAContext }{ }^{*} \text { ctx, int pop, } \\
\text { double }\left({ }^{*} \text { f)(PGAContext }{ }^{*} \text {, int, int), MPI_Comm comm) }\right.\end{array}$ \\
\hline void & PGAFitness(PGAContext ${ }^{*} \operatorname{ctx}$, int popindex) \\
\hline int & PGAGetBestIndex(PGAContext *ctx, int pop) \\
\hline int & PGAGetBinaryAllele(PGAContext ${ }^{*} \mathrm{ctx}$, int $\mathrm{p}$, int pop, int $\mathrm{i}$ ) \\
\hline double & PGAGetBinaryInitProb(PGAContext ${ }^{*} \mathrm{ctx}$ ) \\
\hline char & PGAGetCharacterAllele(PGAContext ${ }^{*}$ ctx, int $p$, int pop, int $\left.\mathrm{i}\right)$ \\
\hline MPI_Comm & PGAGetCommunicator(PGAContext ${ }^{*}$ ctx) \\
\hline double & PGAGetCrossoverProb(PGAContext *ctx) \\
\hline int & PGAGetCrossoverType(PGAContext *ctx) \\
\hline int & PGAGetDataType(PGAContext *ctx) \\
\hline double & PGAGetEvaluation(PGAContext ${ }^{*} \operatorname{ct} x$, int $p$, int pop) \\
\hline int & PGAGetEvaluationUpToDateFlag(PGAContext *ctx, int $p$, int pop) \\
\hline double & PGAGetFitness(PGAContext *ctx, int p, int pop) \\
\hline double & PGAGetFitnessCmaxValue(PGAContext *ctx) \\
\hline int & PGAGetFitnessMinType(PGAContext *ctx) \\
\hline int & PGAGetFitnessType(PGAContext *ctx) \\
\hline int & PGAGetGAIterValue(PGAContext $\left.{ }^{*} \operatorname{ctx}\right)$ \\
\hline int & PGAGetIntegerAllele(PGAContext ${ }^{*}$ ctx, int $p$, int pop, int i) \\
\hline int & PGAGetIntegerFromBinary(PGAContext ${ }^{*} \operatorname{ctx}$, int $p$, int pop, int start, int end) \\
\hline int & PGAGetIntegerFromGrayCode(PGAContext ${ }^{*} \mathrm{ctx}$, int $\mathrm{p}$, int pop, int start, int end) \\
\hline int & PGAGetIntegerInitType(PGAContext *ctx) \\
\hline
\end{tabular}




\begin{tabular}{|c|c|}
\hline Type & Function \\
\hline double & PGAGetMaxFitnessRank(PGAContext *ctx) \\
\hline int & PGAGetMaxGAIterValue(PGAContext *ctx) \\
\hline int & PGAGetMaxIntegerInitValue(PGAContext ${ }^{*}$ ctx, int i) \\
\hline double & PGAGetMaxMachineDoubleValue(PGAContext ${ }^{*}$ ctx) \\
\hline int & PGAGetMaxMachineIntValue(PGAContext *ctx) \\
\hline double & PGAGetMaxRealInitValue(PGAContext *ctx, int i) \\
\hline int & PGAGetMinIntegerInitValue(PGAContext *ctx, int i) \\
\hline double & PGAGetMinMachineDoubleValue(PGAContext *ctx) \\
\hline int & PGAGetMinMachineIntValue(PGAContext *ctx) \\
\hline double & PGAGetMinRealInitValue(PGAContext ${ }^{*}$ ctx, int i) \\
\hline int & PGAGetMutationAndCrossoverFlag(PGAContext ${ }^{*}$ ctx) \\
\hline int & PGAGetMutationBoundedFlag(PGAContext *ctx) \\
\hline int & PGAGetMutationIntegerValue(PGAContext *ctx) \\
\hline int & PGAGetMutationOrCrossoverFlag(PGAContext *ctx) \\
\hline double & PGAGetMutationProb(PGAContext *ctx) \\
\hline double & PGAGetMutationRealValue(PGAContext ${ }^{*} \mathrm{ctx}$ ) \\
\hline int & PGAGetMutationType(PGAContext *ctx) \\
\hline int & PGAGetNoDuplicatesFlag(PGAContext *ctx) \\
\hline int & PGAGetNumProcs(PGAContext ${ }^{*}$ ctx, MPI_Comm comm) \\
\hline int & PGAGetNumReplaceValue(PGAContext *ctx) \\
\hline int & PGAGetOptDirFlag(PGAContext ${ }^{*}$ ctx) \\
\hline double & PGAGetPTournamentProb(PGAContext *ctx) \\
\hline int & PGAGetPopReplaceType(PGAContext *ctx) \\
\hline int & PGAGetPopSize(PGAContext *ctx) \\
\hline int & PGAGetPrintFrequencyValue(PGAContext ${ }^{*}$ ctx) \\
\hline int & PGAGetRandomInitFlag(PGAContext *ctx) \\
\hline int & PGAGetRandomSeed(PGAContext *ctx) \\
\hline int & PGAGetRank(PGAContext ${ }^{*} \mathrm{ctx}$, MPI_Comm comm) \\
\hline double & PGAGetRealAllele(PGAContext ${ }^{*} \mathrm{ctx}$, int $\mathrm{p}$, int pop, int $\left.\mathrm{i}\right)$ \\
\hline double & $\begin{array}{l}\text { PGAGetRealFromBinary(PGAContext *ctx, int } \mathrm{p} \text {, int pop, } \\
\text { int start, int end, double lower, double upper) }\end{array}$ \\
\hline double & $\begin{array}{l}\text { PGAGetRealFromGrayCode(PGAContext *ctx, int } \mathrm{p} \text {, int pop, } \\
\text { int start, int end, double lower, double upper) }\end{array}$ \\
\hline int & PGAGetRealInitType(PGAContext *ctx) \\
\hline double & PGAGetRestartAlleleChangeProb(PGAContext *ctx) \\
\hline int & PGAGetRestartFlag(PGAContext *ctx) \\
\hline int & PGAGetRestartFrequencyValue(PGAContext ${ }^{*}$ ctx) \\
\hline int & PGAGetSelectType(PGAContext $\left.{ }^{*} \operatorname{ctx}\right)$ \\
\hline int & PGAGetSortedPopIndex(PGAContext *ctx, int $\mathrm{n}$ ) \\
\hline int & PGAGetStoppingRuleType(PGAContext *ctx) \\
\hline int & PGAGetStringLength(PGAContext $\left.{ }^{*} \mathrm{ctx}\right)$ \\
\hline double & PGAGetUniformCrossoverProb(PGAContext *ctx) \\
\hline int & PGAGetWorstIndex(PGAContext ${ }^{*}$ ctx, int pop) \\
\hline double & PGAHammingDistance(PGAContext ${ }^{*}$ ctx, int popindex) \\
\hline double & PGAMean (PGAContext ${ }^{*}$ ctx, double ${ }^{*} \mathrm{a}$, int $\left.\mathrm{n}\right)$ \\
\hline int & PGAMutate(PGAContext *ctx, int $\mathrm{p}$, int pop) \\
\hline void & PGAPrintContextVariable(PGAContext *ctx, FILE $\left.{ }^{*} \mathrm{fp}\right)$ \\
\hline void & PGAPrintIndividual(PGAContext ${ }^{*} \operatorname{ctx}$, FILE ${ }^{*} \mathrm{fp}$, int $\mathrm{p}$, int pop) \\
\hline void & PGAPrintPopulation(PGAContext ${ }^{*} \mathrm{ctx}$, FILE ${ }^{*} \mathrm{fp}$, int pop) \\
\hline void & PGAPrintReport(PGAContext *ctx, FILE *fp, int pop) \\
\hline void & PGAPrintString(PGAContext ${ }^{*} \mathrm{ct} x, \mathrm{~F} I L E{ }^{*}$ file, int $\mathrm{p}$, int pop) \\
\hline void & PGAPrintVersionNumber(PGAContext *ctx) \\
\hline
\end{tabular}




\begin{tabular}{|c|c|}
\hline Type & Function \\
\hline double & PGARandom01(PGAContext *ctx, int newseed) \\
\hline int & PGARandomFlip(PGAContext *ctx, double p) \\
\hline double & PGARandomGaussian(PGAContext *ctx, double mean, double sigma) \\
\hline int & PGARandomInterval(PGAContext *ctx, int start, int end) \\
\hline double & PGARandomUniform(PGAContext ${ }^{*}$ ctx, double start, double end) \\
\hline int & PGARank(PGAContext ${ }^{*}$ ctx, int $p$, int ${ }^{*}$ order, int $\left.n\right)$ \\
\hline void & $\begin{array}{l}\text { PGAReceiveIndividual(PGAContext }{ }^{*} \text { ctx, int } \mathrm{p} \text {, int pop, int source, } \\
\text { int tag, MPI_Comm comm, MPI_Status *status) }\end{array}$ \\
\hline void & PGARestart(PGAContext *ctx, int source_pop, int dest_pop) \\
\hline int & PGARound(PGAContext *ctx, double $\mathrm{x}$ ) \\
\hline void & PGARun(PGAContext ${ }^{*} \mathrm{ctx}$, double $\left({ }^{*}\right.$ evaluate)(PGAContext ${ }^{*} \mathrm{c}$, int $\mathrm{p}$, int pop)) \\
\hline void & PGARunGM(PGAContext ${ }^{*} \operatorname{ct} x$, double $\left({ }^{*} \mathrm{f}\right)\left(\right.$ PGAContext ${ }^{*}$, int, int), MPI_Comm comm) \\
\hline void & PGARunMutationAndCrossover(PGAContext *ctx, int oldpop, int newpop) \\
\hline void & PGARunMutationOrCrossover(PGAContext *ctx, int oldpop, int newpop) \\
\hline void & PGASelect(PGAContext *ctx, int popix) \\
\hline int & PGASelectNextIndex(PGAContext *ctx) \\
\hline void & PGASendIndividual(PGAContext ${ }^{*}$ ctx, int $\mathrm{p}$, int pop, int dest, int tag, MPI_Comm comm) \\
\hline void & $\begin{array}{l}\text { PGASendReceiveIndividual(PGAContext }{ }^{*} \text { ctx, int send_p, } \\
\text { int send_pop, int dest, int send_tag, int recv_p, int recv_pop, } \\
\text { int source, int recv_tag, MPI_Comm comm, MPI_Status *status) }\end{array}$ \\
\hline void & PGASetBinaryAllele(PGAContext ${ }^{*} \operatorname{ctx}$, int $\mathrm{p}$, int pop, int $\mathrm{i}$, int val) \\
\hline void & PGASetBinaryInitProb(PGAContext *ctx, double probability) \\
\hline void & PGASetCharacterAllele(PGAContext *ctx, int p, int pop, int i, char value) \\
\hline void & PGASetCharacterInitType(PGAContext *ctx, int value) \\
\hline void & PGASetCommunicator(PGAContext *ctx, MPI_Comm comm) \\
\hline void & PGASetCrossoverProb(PGAContext *ctx, double crossover_prob) \\
\hline void & PGASetCrossoverType(PGAContext ${ }^{*}$ ctx, int crossover_type) \\
\hline void & PGASetDebugLevel(PGAContext ${ }^{*} \operatorname{ctx}$, int level) \\
\hline void & PGASetDebugLevelByName(PGAContext ${ }^{*}$ ctx, char ${ }^{*}$ funcname) \\
\hline void & PGASetEvaluation(PGAContext ${ }^{*} \mathrm{ctx}$, int $\mathrm{p}$, int pop, double val) \\
\hline void & PGASetEvaluationUpToDateFlag(PGAContext ${ }^{*}$ ctx, int $p$, int pop, int status) \\
\hline void & PGASetFitnessCmaxValue(PGAContext ${ }^{*}$ ctx, double val) \\
\hline void & PGASetFitnessMinType(PGAContext *ctx, int fitness_type) \\
\hline void & PGASetFitnessType(PGAContext *ctx, int fitness_type) \\
\hline void & PGASetIntegerAllele(PGAContext ${ }^{*} \operatorname{ct} x$, int $p$, int pop, int $i$, int value) \\
\hline void & PGASetIntegerInitPermute(PGAContext *ctx, int min, int $\max$ ) \\
\hline void & PGASetIntegerInitRange(PGAContext ${ }^{*} \mathrm{ctx}$, int ${ }^{*} \min$, int $\left.{ }^{*} \max \right)$ \\
\hline void & PGASetMaxFitnessRank(PGAContext *ctx, double fitness_rank_max) \\
\hline void & PGASetMaxGAIterValue(PGAContext ${ }^{*}$ ctx, int maxiter) \\
\hline void & PGASetMaxNoChangeValue(PGAContext * ${ }^{*}$ (x, int max_no_change) \\
\hline void & PGASetMaxSimilarityValue(PGAContext ${ }^{*} \mathrm{ctx}$, int max_similarity) \\
\hline void & PGASetMutationAndCrossoverFlag(PGAContext ${ }^{*}$ ctx, int flag) \\
\hline void & PGASetMutationBoundedFlag(PGAContext *ctx, int val) \\
\hline void & PGASetMutationIntegerValue(PGAContext ${ }^{*} \mathrm{ctx}$, int val) \\
\hline void & PGASetMutationOrCrossoverFlag(PGAContext ${ }^{*}$ ctx, int flag) \\
\hline void & PGASetMutationProb(PGAContext *ctx, double mutation prob) \\
\hline void & PGASetMutationRealValue(PGAContext ${ }^{*}$ ctx, double val) \\
\hline void & PGASetMutationType(PGAContext ${ }^{*} \operatorname{ctx}$, int mutation_type) \\
\hline
\end{tabular}




\begin{tabular}{|c|c|}
\hline Type & Function \\
\hline void & $\overline{\left.\text { PGASetNoDuplicatesFlag(PGAContext }{ }^{*} \text { ctx, int no_dup }\right)}$ \\
\hline void & PGASetNumReplaceValue(PGAContext *ctx, int pop_replace) \\
\hline void & PGASetPTournamentProb(PGAContext *ctx, double ptournament_prob) \\
\hline void & PGASetPopReplaceType(PGAContext *ctx, int pop_replace) \\
\hline void & PGASetPopSize(PGAContext *ctx, int popsize) \\
\hline void & PGASetPrintFrequencyValue(PGAContext *ctx, int print_freq) \\
\hline void & PGASetPrintOptions(PGAContext ${ }^{*}$ ctx, int option) \\
\hline void & PGASetRandomInitFlag(PGAContext *ctx, int RandomBoolean) \\
\hline void & PGASetRandomSeed(PGAContext *ctx, int seed) \\
\hline void & PGASetRealAllele(PGAContext ${ }^{*} \mathrm{ctx}$, int $\mathrm{p}$, int pop, int $\mathrm{i}$, double value) \\
\hline void & PGASetRealInitPercent(PGAContext ${ }^{*} \mathrm{ctx}$, double ${ }^{*}$ median, double * percent) \\
\hline void & PGASetRealInitRange(PGAContext ${ }^{*}$ ctx, double ${ }^{*} \min$, double $\left.{ }^{*} \max \right)$ \\
\hline void & PGASetRestartAlleleChangeProb(PGAContext ${ }^{*} \mathrm{ctx}$, double prob) \\
\hline void & PGASetRestartFlag(PGAContext *ctx, int val) \\
\hline void & PGASetRestartFrequencyValue(PGAContext ${ }^{*}$ ctx, int numiter) \\
\hline void & PGASetSelectType(PGAContext *ctx, int select_type) \\
\hline void & PGASetStoppingRuleType(PGAContext *ctx, int stoprule) \\
\hline void & PGASetUniformCrossoverProb(PGAContext *ctx, double uniform_cross_prob) \\
\hline void & PGASetUp(PGAContext ${ }^{*}$ ctx) \\
\hline void & PGASetUserFunction(PGAContext ${ }^{*} \mathrm{ctx}$, int constant, void ${ }^{*} \mathrm{f}$ ) \\
\hline void & PGASortPop(PGAContext *ctx, int pop) \\
\hline double & PGAStddev(PGAContext ${ }^{*} \mathrm{ct} x$, double ${ }^{*} \mathrm{a}$, int $\mathrm{n}$, double mean) \\
\hline void & PGAUpdateGeneration(PGAContext *ctx, MPI_Comm comm) \\
\hline void & PGAUsage(PGAContext ${ }^{*}$ ctx) \\
\hline
\end{tabular}

\section{Fortran 77 Bindings}

Use the rules defined in Chapter 11 (and the machine-specific idiosyncrasies noted in Appendix D) to determine the Fortran bindings. 


\section{Appendix C .}

\section{Parallelism Background}

\section{Parallel Computer Taxonomy}

Traditionally, parallel computers are classified according to Flynn's taxonomy [4]. Flynn's classification distinguishes parallel computers according to the number of instruction streams and data operands being computed on simultaneously.

Flynn's single-instruction single-data (SISD) model is the traditional sequential computer. A single program counter fetches instructions from memory. The instructions are executed on scalar operands. There is no parallelism in this model.

In the single-instruction multiple-data (SIMD) model there is again a single program counter fetching instructions from memory. However, now the operands of the instructions can be one of two types: either scalar or array. If the instruction calls for execution involving only scalar operands, it is executed by the control processor (i.e., the central processing unit fetching instructions from memory). If, on the other hand, the instruction calls for execution using array operands, it is broadcast to the array of processing elements. The processing elements are separate computing devices that rely upon the control processor to determine the instructions they will execute.

In a multiple-instruction multiple-data (MIMD) computer there exist multiple processors each of which has its own program counter. Processors execute independently of each other according to whatever instruction the program counter points to next. MIMD computers are usually further subdivided according to whether the processors share memory or each has its own memory.

In a shared-memory MIMD computer both the program's instructions and the part of the program's data to be shared exist within a single shared memory. Additionally, some data may be private to a processor and not be globally accessible by other processors. The processors execute asynchronously of each other. Communication and synchronization between the processors are handled by having them each read or write a shared-memory location.

A distributed-memory MIMD computer consists of multiple "nodes." A node consists of a processor, its own memory, a network interface, and sometimes a local disk. The program instructions and data reside in the node's memory. The nodes are connected via some type of network that allows them to communicate with each other. Parallelism is achieved by having each processor compute simultaneously on the data in its own memory. Communication and synchronization are handled by passing of messages (a destination node address and the local data to be sent) over the interconnection network.

\section{Processes vs. Processors}

We distinguish the two terms process and processor. A process is a software abstraction with a unique address space that can be scheduled by the operating system. A processor is the physical computer hardware on which computations take place.

On MIMD parallel computers, usually one process executes on each processor (although this is not required). On a uniprocessor, multiple processes timeshare the single processor. 


\section{Message-Passing Programming Model}

In the message-passing programming model multiple processes communicate by passing messages-transferring data from the address space of one process into the address space of another process. When a process needs data stored in the memory of another process, the data must be sent from the process that "owns" it, to the memory of the process that needs it.

The message-passing programming model is currently one of the most popular. One reason for the popularity is portability. Message passing is the natural programming model on distributed-memory MIMD computers. Each process is naturally mapped to one of the machine's nodes. A similar implementation is common on a workstation network where one process runs on each workstation. On a shared-memory MIMD computer multiple processes can emulate message passing by communicating only via logical message queues-areas of shared memory partitioned by process. On a uniprocessor the multiple processes that timeshare the physical processor can also emulate the idea of logical message queues for their communication.

One example of the message-passing programming model is the master/slave model. In this model a master process distributed work (computation to be performed) to the slave processes. The slaves perform the work and return the result to the master. In many implementations the master plays a bookkeeping role only and does not perform any computation.

\section{Parallel Genetic Algorithms}

When using the term "parallel genetic algorithm" it is important to distinguish between parallel models, their (parallel or sequential) implementation, and the computer hardware.

\section{Models}

A sequential GA model (more accurately called a global model) has a single population and no restrictions (partitioning) upon which strings recombine with which. The sequential GA is the traditional GA model given in the literature. In a parallel GA model there are either multiple populations (an island model) or a partitioning of a single population (often called a fine-grained model).

\section{Implementations}

Both parallel and sequential GA models can have parallel or sequential implementations. A sequential implementation of the global model is the traditional approach discussed in the GA literature. One process, running on a uniprocessor (PCs and workstations), performs all the calculations. In a parallel implementation of the global model the steps of the GA (some or all of selection, crossover, mutation, and fitness calculation) are executed simultaneously by multiple processes running on a parallel computer or workstation network.

In a sequential implementation of a parallel GA model, multiple processes, each responsible for a subpopulation or partition of the full population, time share the processor of the uniprocessor they are running on. In a parallel implementation of a parallel GA model, the multiple processes each run on a unique processor of a parallel computer or workstation network.

\section{MPI}

MPI (Message Passing Interface) is a specification of a message-passing library for parallel computers and workstation networks-it defines a set of functions and their behavior. The actual implementation of this interface is left up to vendors and researchers to develop. At the time of this writing several implementations of MPI, both proprietary and freely available, exist. MPI was designed by a large group of parallel computer vendors, computer researchers, and application developers as a standard for message passing. 


\section{Communicators}

Almost all MPI functions require a communicator. If MPI routines are called directly, the user must supply a communicator. Also, if any of PGAPack's parallel routines, other than PGARun, are used, the user must supply a communicator as well.

A communicator combines the notions of context and group. A context is an extension of the notion of a "tag" used in many other message-passing systems to identify a message. Contexts differ from tags in that they are allocated by the system, not the user, and that no wild-card matching among contexts is allowed. A group contains $n$ processes whose rank is an integer between $0, \ldots, n-1$. Processes may belong to more than one group and have a unique rank within each.

Any MPI implementation will always supply the default communicator MPI_COMM_WORLD. This communicator contains all processes that were created when MPI was initialized. For most users this is all they have to know about communicators. Simply supply MPI_COMM_WORLD whenever a communicator is required as an argument. For more sophisticated use, users are referred to $[5,6]$.

\section{Parallel I/O}

The issue of parallel I/O is independent of PGAPack. However, since we assume many PGAPack users will wish to do $\mathrm{I} / \mathrm{O}$ we address this topic. A primary consideration has to do with whether one or all processors do I/O. Consider the following two code fragments, keeping in mind that they are being executed simultaneously by multiple processes:

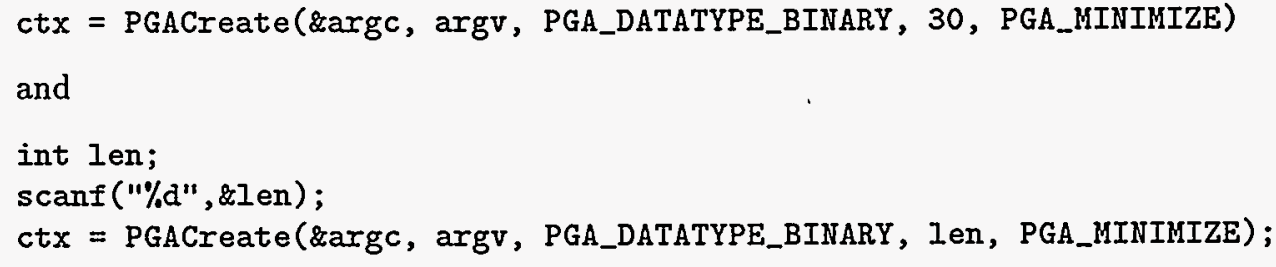

In the first case, all processes will receive the value of 30 for the string length since it is a constant. In the second case, however, the value of the string length is determined at run time. Whether one or all processes execute the scanf function is undefined in MPI and depends on the particular parallel computing environment. In PGAPack we require that all processes have the same values for all fields in the context variable. Since some of these fields may be set by using values specified at run time, we suggest that your I/O that reads in PGAPack parameters be done as follows:

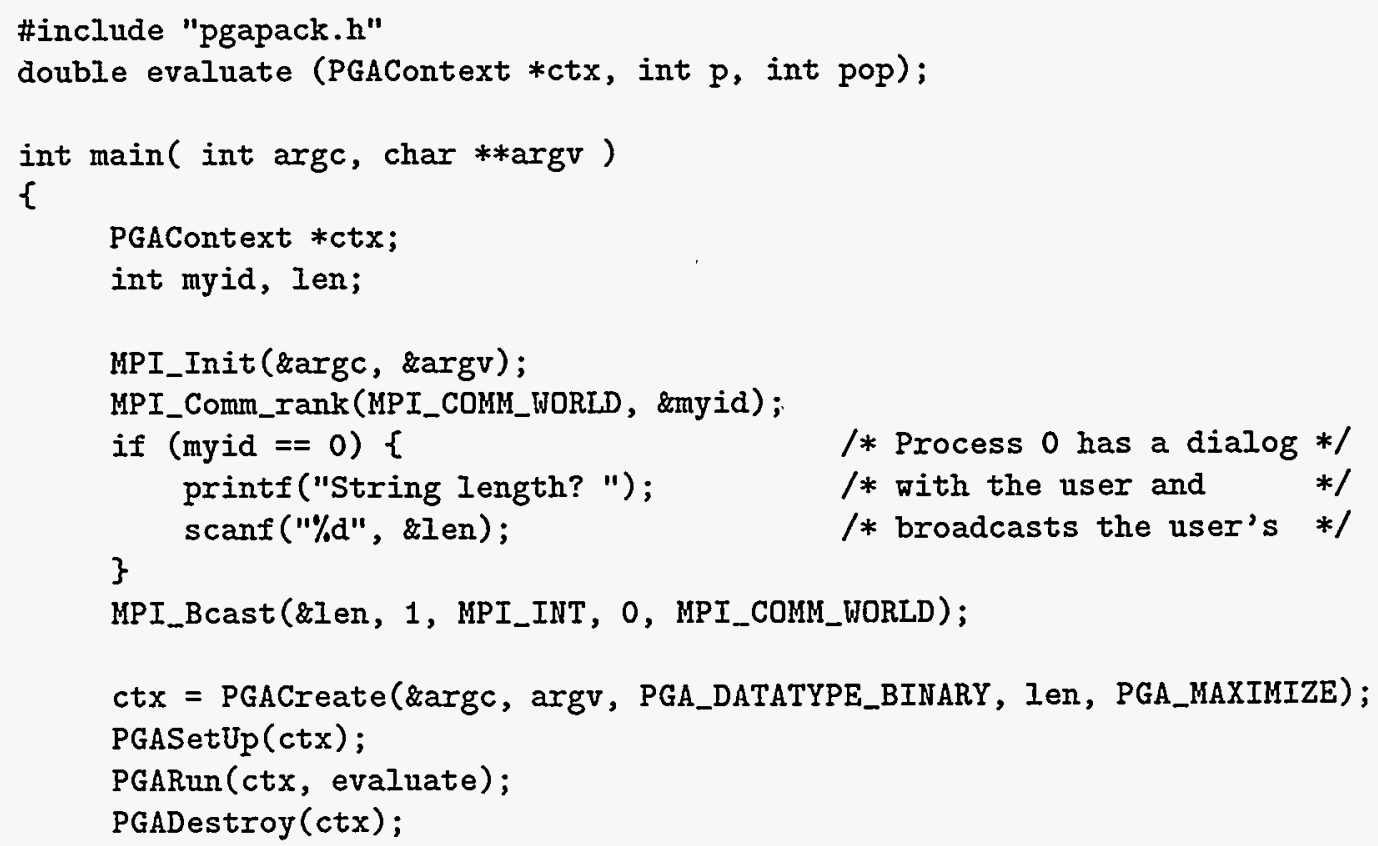


MPI_Finalize();

return $(0)$;

\}

The key point is that only process 0 (as determined by MPI_Comm_rank) performs I/O and the value of len is then broadcast (using MPI_Bcast) to the other processes. 


\section{Appendix D}

\section{Machine Idiosyncrasies}

\section{Data Type Sizes}

PGAPack is written entirely in ANSI C. However, because it is callable from Fortran, and no standards exist for interlanguage communication, problems may arise. These have to do with a lack of consistency in the size of data types between the two languages.

On all machines we have tested, an integer declaration in Fortran is the same size as an int declaration in $\mathrm{C}$ and everything works properly. For floating-point numbers, however, we have found at least one inconsistency. The requirement is for the Fortran floating-point number to be the same size as a $\mathrm{C}$ double. On most machines a Fortran double precision declaration is the equivalent size. On the Cray T3D, however, by default, the Fortran data type double precision is not supported and must be handled as described below.

Since Fortran does not support pointers, an integer variable is used to hold the address of the context variable (and possibly MPI communicator addresses as well). Therefore, a Fortran integer must be "large enough" to hold an address on the machine. For all 32-bit address space machines we have tested this is the case. On machines with a 64-bit address space, however, this may not be true. In particular, the size of a Fortran integer on the Silicon Graphics Power Challenge and DEC Alpha (but not the Cray T3D) is 32-bits and is not large enough to hold a machine address. The solution on these machines is to use the (nonstandard, but supported) Fortran declaration integer*8 for the context variable.

\section{Startup}

The MPI standard provides for source code portability. However, the MPI standard does not specify how an MPI program shall be started or how the number of processes in the computation is specified. These will vary according to the computer being used and the choice of MPI implementation. The notes below are from our experiences testing PGAPack on different machines.

\section{Silicon Graphics Challenge}

The Silicon Graphics Challenge is a 32-bit symmetric multiprocessor. We used MPICH with the ch_shmem device and the ncc $\mathrm{C}$ compiler. Several warnings were received

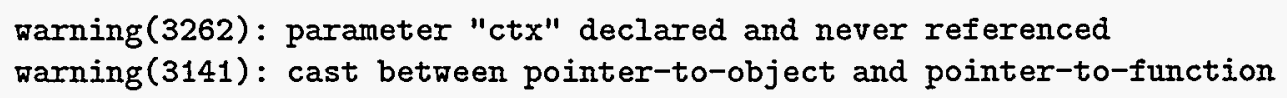

but the library was successfully built. To run a parallel PGAPack program, use either

a.out -np nprocs

or MPICH's mpirun command. 


\section{Silicon Graphics Power Challenge}

The Silicon Graphics Power Challenge is similar to the Challenge, except that it has a 64-bit address space. On this machine the size of an integer (int in $\mathrm{C}$ and integer in Fortran) is not the same as the size of an address. Fortran users should use the declaration integer $* 8$ for the context variable (and integer for other Fortran integer declarations). See also Chapter 11.

We used MPICH with the ch_p4 device and the the MIPSpro C compiler (cc). We found a bug in pca, the Power C Analyzer, and recommend not using it for now. (To do this do not specify the -pca switch to cc). To run a parallel PGAPack program, use

a.out -np nprocs

or HPICH's mpirun command.

\section{Cray T3D}

The Cray T3D has a 64-bit address space. However, the size of an integer on the T3D is the same as the size of an address, and therefore no special considerations are needed for declaring the context variable in Fortran.

On the T3D a C double is 64 bits. The Fortran double precision data type, however, is not supported (by default). One workaround is to declare all floating-point numbers REAL, as these are 64 bits on the T3D. The other workaround is to use the compiler switch "-dp".

To compile for a Cray T3D, cross compilation is done on a front-end machine (a Cray C90 in our case). Set Cray's TARGET environment variable so the compiler, linker, etc., will know which architecture to compile for.

setenv TARGET cray-t3d

An alternative is to use "-T cray-t3d" with $c c$ and "-C cray-t3d" with cf77. Another alternative is to explicitly use the cross compilers (/mpp/bin/cc and /mpp/bin/cf77) and linker (/mpp/bin/mppldr).

We used the MPI in /usr/local/mpp/lib/libmpi.a. Adding - Impi in your link step may also find the MPI library. If a successful T3D executable was built, the command "file a.out" should say "MPP absolute."

To run a parallel PGAPack program, use

a. out -npes nprocs

where nprocs is a power of two.

\section{Intel Paragon}

We used MPICH with the $c h . n x$ device and compiled with $c c-n x$. To run a parallel PGAPack program, use

a.out -sz nprocs

or MPICH's mpirun command.

\section{IBM SP2}

We tested the IBM SP2 using both MPICH with the ch_eui device, and IBM's research MPI, MPI-F. We compiled PGAPack with $x I c$ and linked with mpCC. Execution required setting a number of environment variables. We were successful with the following, but this may vary with the system software installed on the SP you are using.

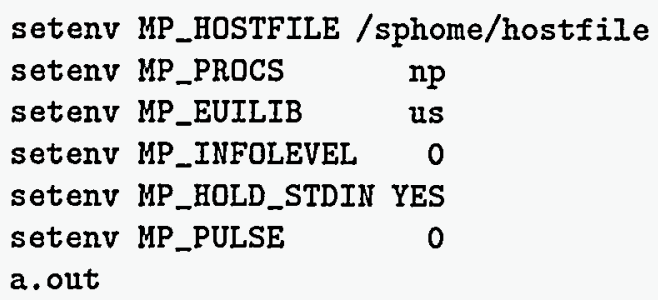




\section{Convex Exemplar}

We used MPICH with the ch_shmem device. Be sure to compile (the Fortran examples) with fort77, not \pm 77. Also, you must link with /usr/lib/Iibu77. a last to satisfy iargc and getarg. This must be done manually in the prototype makefiles ./examples/fortran/Makefile.in and ./examples/mgh/Makefile.in before running configure. To run a parallel PGAPack program using MPICH use the mpirun command.

\section{Sun SparcStation}

We used MPICH with the ch_p4 device and the GNU C compiler gcc. The instverf test program was run using 4 processes with:

/usr/local/mpi/bin/mpirun instverf -arch sun4 -np 4

\section{Silicon Graphics Workstation}

We used MPICH with the ch_p4 device and mpirun command, the cc $\mathrm{C}$ compiler, and $f 77$ Fortran compiler.

\section{IBM/RS6000 Workstation}

We have successfully run PGAPack on both single workstations and networks of workstations using the MPICH implementation with the ch_p4 device.

\section{Hewlett Packard Workstation}

We used MPICH with the ch_shmem device and mpirun command, the gcc C compiler, and fort77 Fortran compiler.

\section{DEC Alpha Workstation}

DEC Alpha workstations have a 64-bit address space. On this machine the size of an integer (int in C and integer in Fortran) is not the same as the size of an address. Fortran users should use the declaration integer*8 for the context variable (and integer for other Fortran integer declarations). See also Chapter 11. 


\section{Appendix E}

\section{Common Problems}

- When reading input value to be used as parameters in PGASet calls, the PGAset calls themselves may not be executed until after PGACreate has been called.

- In $\mathrm{C}$, when reading input parameters which are of type double, the scanf conversion specification should be of the form \% If, not \% which is appropriate for floats.

- An infinite loop can occur if the number of permutations of the bit string is less than the population size. For example, for a binary-valued string of length four, there are $2^{4}=16$ possibilities. If the population size is greater than 16 , and duplicate strings are not allowed in the population, an infinite loop will occur.

- Erroneous results can occur if the name of a user's function conflicts with a library function used by PGAPack. For example, if a program defined its own ceil function, this would conflict with the C math library function of the same name.

- All floating point constants and variables used in PGAPack are of type double. Particularly from Fortran, the user should be careful to make sure that they pass a double precision constant or variable.

- PGACreate removes command line arguments. One consequence is that if PGACreate is called twice in the same program (unusual, but legal), the second PGACreate call will not receive the command-line arguments.

- If one includes mpi.h (or mpif.h) when it should not be, errors will result, as well as warnings about 'redefining macros and typedefs. This usually happens when a sequential version of PGAPack is used (with "fake" MPI stub routines and definitions) and the user's program explicitly includes "real" mpi.h or mpif.h header files.

- If one fails to include mpi.h (or mpif.h) when it should be (such as calling MPI functions directly) errors may result. Since pgapack. $h$ includes mpi.h this should not happen in C. The Fortran include file, pgapackf.h, however, does not include mpif.h. The user must explicitly include it in every subroutine and function that makes MPI calls. Not including mpif.h could result in any of several different errors, including

- syntax errors when compiling (for example, MPI_COMM_WORLD being undefined)

- general errors in the computed results

- the program crashing when it calls the undefined subroutine MPI_Init

- general MPI errors such as:

o - Error in MPI_COMM_RANK : Invalid communicator

[0] Aborting program! 
We have also seen the following error from not including bmpif.h in the main program:

PGACreate: Invalid value of datatype: 0

PGAError: Fatal

- If the ch-p4 device in MPICH is used to run on workstations one must have a correct processor group file (procgroup). The error message

(ptera-36\%)a.out

p0_18429: p4_error: open error on procgroup file (procgroup): 0

(ptera-37\%)

may occur if the processor group file is not specified correctly. See the MPICH users guide for more details.

- A common error with the procgroup file when using the ch_p4 device in MPICH is to have an incorrect path to the executable.

-When compiling the examples directory we have seen "multiply defined" error messages. For example:

Making C examples

Compiling classic

ld: /usr/local/mpi/lib/sun4/ch_p4/Iibmpi.a(initialize.o): _MPI_Initialized: multiply defined collect2: Id returned 2 exit status

We have seen this error occur when a sequential version of PGAPack was built and the library (./lib/arch/libpgag.a or ./lib/arch/libpga0.a) was not deleted before attempting to build a new, parallel version of PGAPack. The "fake" MPI stub routines are in the sequential library and have name conflicts when a "real" MPI library is referenced. The solution is to delete the old .a file and rerun make install. 


\section{Acknowledgments}

Much of the code in PGAPack was originally developed as part of the author's Ph.D. thesis. Significant contributions to the development of PGAPack were made by Philip Hallstrom, David Noelle, Greg Reeder, and Brian Walenz, participants in Argonne's Science and Engineering Research Semester program.

Many aspects of PGAPack-including the user interface, choice of some data structures, and design of Fortran wrappers-were strongly influenced by the design of the PETSc (Portable and Extensible Tools for Scientific Computing) library. I thank Bill Gropp, Lois Curfman McInnes, and Barry Smith for many helpful discussions. The code in PGAPack for parsing command line arguments is a modified version of that used in the p4 system developed by Ralph Butler and Rusty Lusk. 


\section{Bibliography}

[1] MPICH World Wide Web home page, June 1995. Available by anonymous ftp from ftp.mcs.anl.gov in directory pub/mpi, file mpich.tar.z, or on the World Wide Web at http://wพw.mcs.anl.gov/home/lusk/mpich/index.html.

[2] J. Baker. Reducing bias and inefficiency in the selection algorithm. In J. Grefenstette, editor, Proceedings of the Second International Conference on Genetic Algorithms and Their Applications, pages 14-21, Hillsdale, New Jersey, 1987. Lawrence Erlbaum Associates.

[3] L. Davis. Handbook of Genetic Algorithms. Van Nostrand Reinhold, New York, 1991.

[4] M. Flynn. Some computer organizations and their effectiveness. IEEE Transactions on Computers, 21:948-960, 1972.

[5] Message Passing Interface Forum. MPI: A message-passing interface standard. International Journal of Supercomputing Applications, 8(3/4), 1994.

[6] W. Gropp, E. Lusk, and A. Skjellum. USING MPI Portable Parallel Programming with the MessagePassing Interface. The MIT Press, Cambridge, 1994.

[7] J. Holland. Adaption in Natural and Artificial Systems. MIT Press, Cambridge, 1992.

[8] W. Spears and K. DeJong. On the virtues of parameterized uniform crossover. In R. Belew and L. Booker, editors, Proceedings of the Fourth International Conference on Genetic Algorithms, pages 230-236, San Mateo, 1991. Morgan Kaufmann.

[9] G. Syswerda. Uniform crossover in genetic algorithms. In J. Schaffer, editor, Proceedings of the Third International Conference on Genetic Algorithms, pages 2-9, San Mateo, 1989. Morgan Kaufmann.

[10] D. Whitley. The GENITOR algorithm and selection pressure: Why rank-based allocation of reproductive trials is best. In J. Schaffer, editor, Proceedings of the Third International Conference on Genetic Algorithms, pages 116-121, San Mateo, 1989. Morgan Kaufmann.

[11] D. Whitley and J. Kauth. GENITOR: A different genetic algorithm. In Rocky Mountain Conference on Artificial Intelligence, pages 118-130, Denver, 1988. 
Distribution for ANL-95/18

Internal:

J. K. Beumer (10)

F. Y. Fradin

W. D. Gropp

D. M. Levine (10)

L. C. McInnes

G. W. Pieper

B. F. Smith

F. J. Stevens

R. L. Stevens

C. L. Wilkinson

TIS File

\section{External:}

DOE-OSTI, for distribution per UC-405 (52)

ANL-E Library

ANL-W Library

Manager, Chicago Operations Office, DOE

Hathematics and Computer Science Division Review Committee:

F. Berman, University of California at LaJolla

G. Cybenko, Dartmouth College

T. DuPont, The University of Chicago

J. G. Glimm, State University of New York at Stony Brook

M. T. Heath, University of Illinois, Urbana

E. F. Infante, University of Minnesota

K. Kunen, University of Wisconsin at Madison

R. E. O'Malley, University of Washington

L. R. Petzold, University of Minnesota

F. Howes, Dept. of Energy - Office of Computational and Technology Research

D. Nelson, Dept. of Energy - Office of Computational and Technology Research 